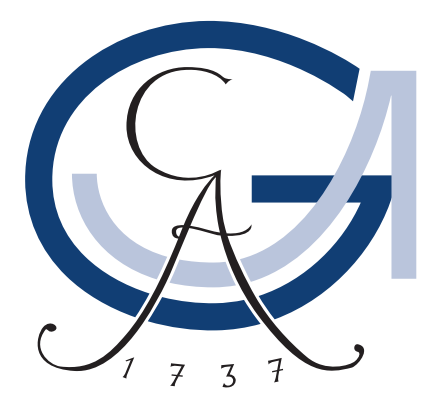

\title{
Investigation of voltage- and light-sensitive ion channels
}

\section{Dissertation}

for the award of the degree

"Doctor rerum naturalium"

of the Georg-August Universität Göttingen

within the doctoral program

Physics of Biological and Complex Systems

of the Göttingen Graduate School for Neuroscience, Biophysics and Molecular Biosciences (GGNB)

of the Georg-August University School of Science (GAUSS)

\author{
submitted by \\ Ulrich Fromme \\ from Engelskirchen, Germany
}

Göttingen, August 1, 2016 
Prof. Dr. Christoph F. Schmidt ( $1^{\text {st }}$ reviewer and supervisor)

Drittes Physikalisches Institut

Georg-August Universität Göttingen

Dr. Andreas Neef ( $2^{\text {nd }}$ reviewer)

Max Planck Institute for Dynamics and Self-Organization/

Bernstein Center for Computational Neuroscience Göttingen

Prof. Dr. Tobias Moser

Institute for Auditory Neuroscience

University Medical Center Göttingen

Additional members of the examination committee:

Prof. Dr. Florentin Wörgötter

Drittes Physikalisches Institut/ BCCN

Georg-August Universität Göttingen

Prof. Dr. Stefan Klumpp

Institut für Nichtlineare Dynamik

Georg-August Universität Göttingen

Prof. Dr. Marc Timme

Network Dynamics

Max Planck Institute for Dynamics and Self-Organization Göttingen

Date of the oral examination: February $29^{\text {th }}, 2016$ 


\section{Affidavit}

I herewith declare that I have produced this dissertation without the prohibited assistance of third parties and without making use of aids other than those specified. Notions taken over directly or indirectly from other sources have been identified as such. This dissertation has not previously been presented in identical or similar form to any other German or foreign examination board.

This thesis work was conducted from January 2012 to December 2015 under the supervision of Prof. Dr. Christoph F. Schmidt at the Third Institute of Physics Biophysics.

Ulrich Arthur Fromme

Göttingen, August 1, 2016 


\section{Contents}

\begin{tabular}{lll}
\hline 1 & Introduction & 1
\end{tabular}

2 Basics of electrophysiology 3

2.1 Recording ion currents . . . . . . . . . . . . . . . 3

2.2 The cell membrane equivalent circuit . . . . . . . . . . . . . 4

2.3 The patch-clamp method . . . . . . . . . . . . . . . . . . 7

2.4 The patch-clamp amplifier . . . . . . . . . . . . . . . . . 10

3 Scanning lon Conductance Microscopy (SICM) 13

3.1 The principle of SICM . . . . . . . . . . . . . . . . . . . 13

3.2 Investigation of the abscission process during cell division . . . . . . 18

4 Electrophysiology/ SICM in neuroscience 23

4.1 Ion channels as the basis of neural activity . . . . . . . . . . . . . . 24

4.2 Transmission speed of spike-rate coded signals . . . . . . . . . . . . . . 27

4.3 Action potential initialization . . . . . . . . . . . . . . 29

$4.4 \quad$ Electrophysiologic measurements at the axon initial segment . . . . . 30

\begin{tabular}{|lll}
5 & Characterization of the channelrhodopsins Chronos and ChR2 & 37
\end{tabular}

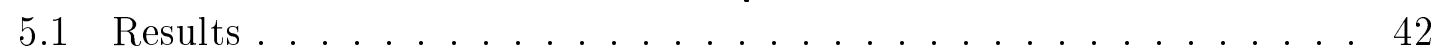

5.1 .1 Time constants of the current waveform response . . . . . . 42

5.1 .2 Early intermediates of the light cycle . . . . . . . . . . . . 42

5.1.3 Challenges for a complete model/effects of long pulses. . . . . 47

5.1 .4 Evaluation via simulation using a complete model . . . . . . . 50

$5.1 .5 \quad$ Influence of single transitions on waveform . . . . . . . . . . 51

$5.1 .6 \quad$ Voltage dependence of Chronos . . . . . . . . . . . . . . . 54

$5.1 .7 \quad$ High-intensity deactivation . . . . . . . . . . . . . . 55

5.1 .8 Comparison to spectroscopy and other models . . . . . . . . 57

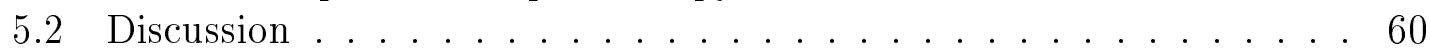

6 Experimental/theoretical procedures $\quad 62$

6.1 Materials and Methods . . . . . . . . . . . . . . 62

6.1 .1 Pipettes . . . . . . . . . . . . . . . . 62

6.1 .2 Setup design . . . . . . . . . . . . . . . . . . . . . . . . . . . . 67

$6.1 .3 \quad$ Measurement protocols . . . . . . . . . . . . . . . . . . 73

6.1 .4 Cell biology . . . . . . . . . . . . . . . . . . . . . . . . . . . . 75

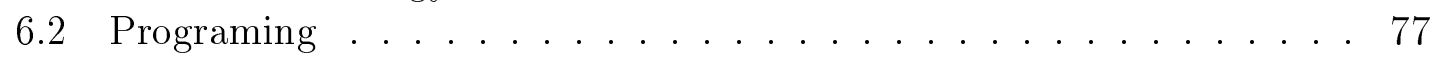

6.2 .1 Visualization of SICM images . . . . . . . . . . . 77

6.2 .2 Markov models . . . . . . . . . . . . . . . 83 
$6.2 .3 \quad$ Finding optimal parameters . . . . . . . . . . . . . 84

6.2 .4 Data analysis $\ldots \ldots \ldots \ldots . \ldots . \ldots . \ldots 89$

\begin{tabular}{lll}
\hline 7 & Summary and Outlook & 92
\end{tabular}

\begin{tabular}{ll}
\hline List of Figures & 94
\end{tabular}

\begin{tabular}{ll}
\hline Abbreviations & 96
\end{tabular}

\begin{tabular}{ll}
\hline Bibliography & 98
\end{tabular}

\begin{tabular}{ll}
\hline Acknowledgments & 108
\end{tabular} 


\section{Introduction}

Understanding the operation of the brain and thus the basis of thinking has been a long desired goal for centuries. The discovery that electric currents form the foundation of the nervous system, as demonstrated by Galvani in 1791, has opened a new path to investigate this system. Since then, numerous studies have been performed in the field of electrophysiology i.e. the measurement of ionic currents in biological systems or the changes in electric potential caused by them in order to gain insights into neural networks and even find cures for neural disorders.

As in most natural sciences, new insights are often coupled or preceded by the developments of new equipment and techniques. In electrophysiology many of the groundbreaking advances are closely connected to an increase of the signal-to-noise ratio enabling the investigation of smaller currents created by finer structures.

Hodgkin and Huxley showed that variable ion selectivity of the cell membrane is responsible for the creation of electric signals in living cells by using voltage clamp with sensitive equipment during the first half of the twentieth century [1]. Neher and Sackmann managed to record single channel currents thanks to their ultra low-noise patch-clamp configuration nearly thirty years later [2].

Progress has not only been made in the design of better equipment and measuring techniques but also in the field of evaluating data [3]. Modern computers have facilitated the integration and cross correlation of large amounts of data. In neuroscience this is especially visible in the field of computational neuroscience, dealing with the simulation of large neural networks [4]. With the bottom up approach of simulating large systems based on the properties or simplified principles of single cells, tackling the complexity of large neural systems is possible. The most elaborate attempt in this regard is the "Human Brain Project 11 attempting to create a virtual representation of an entire brain.

Data analysis also profits considerably from these advances. Advanced spike sorting algorithms enable the analysis of recordings from multiple neurons with a single electrode on a large scale by assigning each spike to the cell it came from [5]. While recordings and correlations from hundreds of neurons in modern multi-electrode setups [6, 7] cannot be thoroughly investigated without digital signal storage and evaluation [8].

The same is true for long time measurement requiring complicated and precise control of the stimulation inputs [9]. Many of these measurements would be incredibly difficult to impossible without the use of modern computers.

One of the goals of this thesis was to create an experimental procedure able to investigate the electrical membrane properties in the region of the axon initial segment (AIS) in cortical neurons. The AIS is a delicate structure responsible for action

\footnotetext{
${ }^{1}$ https://www.humanbrainproject.eu/
} 
potential initiation and its properties strongly influence the information processing in the brain. Traditional measurements on this structure have provided conflicting results due to their invasiveness [10]. To reduce the invasiveness of recordings while achieving highly localized and precise information on the membrane properties, this study combined a topological measurement using 'scanning ion conductance microscopy' (SICM) with highly sensitive extra-cellular recordings. Using a customized simulation software developed by Andres Agudelo-Toro [11, the topographic and electrophysiological information can be combined to infer information about the gating behavior and density of sodium channels.

A detailed description of the SICM method is given in chapter 3. A side project dealing with the investigation of 'midbodies' is used to elucidate the strengths and limitations of the technique. The 'midbody' is a structure connecting two freshly divided cells and is not anchored to any surface, which makes it ideal to show the force-free scanning of SICM.

Chapter 4 explains in detail how properties of the AIS influence signal processing in the brain, and shows how SICM in combination with extra-cellular recordings can be used for investigating those properties.

In Chapter 5 an extensive characterization of the light-gated ion channel 'Chronos' is performed. This channel is a promising tool for low-invasive neural stimulation due to its unrivaled kinetics.

During the course of this work, substantial improvements to the existing SICM system were performed. Among the most important is the integration of an external patch-clamp amplifier enabling the combination of topographic images with lownoise current- and voltage-clamp measurements, the addition of laser illumination for well defined high-intensity illumination and the addition of a second recording electrode for the simultaneous recording of two distinct signals. On the post-recording side, major improvements have been achieved due to custom software for analysis of both topographical and electrophysiological data. A thorough investigation of the used tools was performed including the characterization of the SICM probes and the optogenetic tools to increase the control over the system.

The investigation of the light-gated ion channel 'Chronos' has yielded especially interesting results, as I was not only able to create a detailed Markov model reflecting the channel behavior but also infer information about the structure/function relationship. 


\section{Basics of electrophysiology}

\subsection{Recording ion currents}

In conventional electronic circuits comprising metal and semiconductor parts, all charge of the electrical current is carried by electrons $\left(e^{-}\right)$. In ionic solutions on the other hand, electric charge is carried by at least two kinds of ions which can possess different charge and mobility. As all the measuring equipment is based on metal or semiconductor parts, a good conversion of electron currents to ionic currents is needed. This conversion can be achieved by electrodes consisting of different materials such as platinum or tungsten. In this study however the conversion is performed at a silver/silver-chloride interface. At these electrodes, conversion between $e^{-}$and $\mathrm{Cl}^{-}$takes place via the reversible reaction given in equation 2.1.1, so that current flow is possible without polarization of the electrode or creating local $\mathrm{pH}$ changes.

$$
\mathrm{Ag}+\mathrm{Cl}^{-} \rightleftharpoons \mathrm{AgCl}+e^{-}
$$

A millimeter sized pellet of compressed $\mathrm{AgCl}$ submerged directly in the bath solution was used as a low-resistance reference electrode while a silver wire that was dipped into molten $\mathrm{AgCl}$ was used as the recording electrode. For small currents not surpassing several nanoampere, $\mathrm{Ag} / \mathrm{AgCl}$ electrodes can record voltages accurately even while passing current, provide a stable signal and are non poisonous as long as the silver-chloride coating of the silver wire is present [12] . These electrodes do however require $\mathrm{Cl}^{-}$in the solution, and the half-cell potential depends on the $\mathrm{Cl}^{-}$ concentration at the electrode. For the reference electrode this only causes problems if the concentration in the bath solution is changing, which was of no large concern in my experiments. (Agar bridges can be used to separate the solution surrounding the pellet from the bath, to remove this effect completely.) For the recording electrode however this could cause a problem, as the concentrations in tissue and cells differ from that of the bath and are hard to control. This is one of the reasons the recording electrodes are placed in solution-filled glass pipettes, keeping the $\mathrm{Cl}^{-}$ concentration at the solution/electrode interface stable.

Even if acceptable electron-to-ion conversion is achieved however, current measurements in electrophysiological recordings face certain complications usually not encountered in traditional electronics. This is due to the properties of charge flow in ionic solutions. While the basic laws of electronics such as Ohm's and Kirchhoff's laws still apply, the presence of multiple different charge carriers and the lack of limited current pathways (wires) often make the application of such laws less intuitive.

In the following, some of the effects relevant to the performed measurements are addressed as well as established methods to counteract these issues. 


\subsection{The cell membrane equivalent circuit}

Current and voltage measurements performed at the membranes of electrically active cells give important information about the processes underlying their behavior. To properly interpret these measurements and be aware of their limitations, some concepts about the electrical properties of membranes and the associated ion currents have to be considered. An equivalent electronic circuit representation of the cell membrane makes it possible to mathematically characterize the electronic effects rather easily. In such a model the membrane is represented by a resistance in parallel with a capacitance as shown in Figure 2.1 (B). The sum of ion channel conductances $G_{i}$ determines the resistance $\mathrm{R}$ in this model in the following way:

$$
R^{-1}=G_{\text {total }}=\sum_{i} G_{i}
$$

The dielectric properties of the lipid bilayer lead to the creation of a local plate capacitor with a specific capacitance $C_{s} \approx 1 \mu \mathrm{F} / \mathrm{cm}^{2}$. Integrating over the entire membrane area results in the total capacitance

$$
C=\int_{A} C_{s} d A \approx C_{s} A .
$$
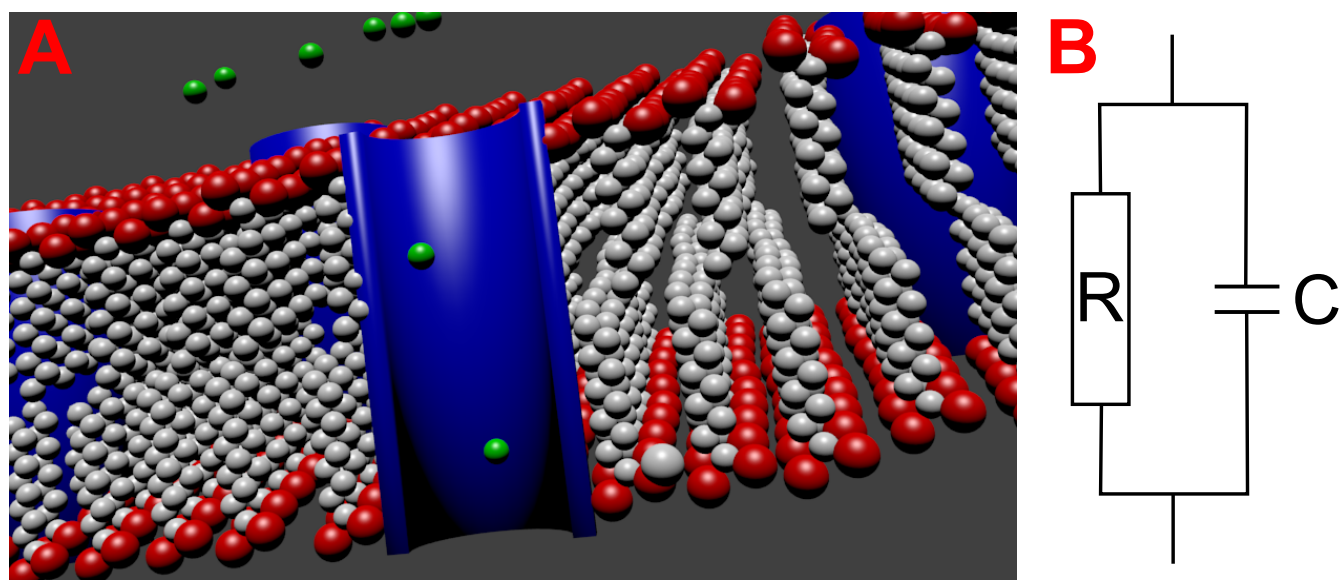

Figure 2.1: The cell membrane as a circuit. A: The membrane of a cell possesses both: conducting elements $G_{i}$ due to its ion channels (shown in blue) as well as a capacitance due to the dielectric quality of its lipid bilayer. The sum of these elements give rise to a global resistance $R$ in parallel with a global capacitance $C$. B: An equivalent circuit of a single cell membrane.

This parallel arrangement of ohmic and capacitive resistances also leads to two kinds of ionic currents across the membrane. One is the current passing through conducting elements while the other consists of charging or un-charging the capacitor. The most obvious effect produced by this circuit is the large transient current $I_{C}$ arising from the capacitor during a change in the membrane voltage $V_{m}$. The capacitive current in such an event is given by 


$$
I_{C}=C \frac{d V_{m}}{d t}
$$

If during an experiment a voltage step of $100 \mathrm{mV}$ is applied in $10 \mu$ s for example, this leads to a current of $I_{C}=10.000 \mathrm{~V} / \mathrm{s} \cdot C$ which would mean $10 \mathrm{nA}$ for every picofarad of capacitance. As such a current is several orders of magnitude larger than that of a single channel it would drown the underlying ohmic currents and thus requires compensation as shown in section 2.4 .

A second effect of the resistor/capacitor circuit is the reduction of the current response speed during recording. Compensation of this low-pass filtering proves less effective both at the recording level and during post processing than for the afore mentioned effect. The underlying principle is also slightly less intuitive especially when a constant voltage is applied inside the cell. This is why a detailed mathematical description is provided in the following.

To derive the equation for the low-pass filtering during recording, it is first necessary to take into account that every electrode used to measure ionic currents also has an input resistance (usually called $R_{\text {series }}$ as it is in series with the membrane resistance during recordings). In modern electrophysiological measurements this is mainly determined by the resistance $R_{P}$ of the glass pipette harboring the actual electrode and the access resistance to the opening of this pipette $R_{\text {access }}$. If either no electrode is present or its voltage is equal to the natural membrane potential $V_{r}$, then the ohmic and capacitive currents are equal, which can be written as

$$
I_{O h m}+C \frac{d V_{m}}{d t}=0
$$

This relation can be interpreted as a conservation of charges, since without an external pipette all ions arriving or leaving the capacitor also have to cross the membrane. If an electrode with an applied voltage $V_{c}$ different from the membrane potential is present, the equation changes to

$$
I_{\text {Ohm }}+C \frac{d V_{m}}{d t}=I_{\text {injected }}=\frac{V_{c}-V_{m}}{R_{\text {series }}} .
$$

The current through the ion channels is non-zero at membrane voltages different from the natural potential and is given by $I_{O h m}=\left(V_{m}-V_{r}\right) / R$. Thus the above equation becomes

$$
\frac{V_{m}-V_{r}}{R}+C \frac{d V_{m}}{d t}=\frac{V_{c}-V_{m}}{R_{\text {series }}} \Leftrightarrow V_{m}\left(\frac{1}{R}+\frac{1}{R_{\text {series }}}\right)+C \frac{d V_{m}}{d t}=\frac{V_{c}}{R_{\text {series }}}+\frac{V_{r}}{R} .
$$

Which can be rewritten as

$$
V_{m}+C \frac{R R_{\text {series }}}{R+R_{\text {series }}} \frac{d V_{m}}{d t}=\frac{V_{c} R+V_{r} R_{\text {series }}}{R+R_{\text {series }}} .
$$

To see the consequences of this more clearly, equation 2.2.7 can be simplified to

$$
V_{m}+\tau \frac{d V_{m}}{d t}=V_{\infty}
$$


where $\tau$ indicates the time constant of the approach to a new membrane potential and $V_{\infty}$ represents the value that $V_{m}$ will reach for a certain applied voltage at the electrode. Thus the above equation does not only explain the low-pass filtering of the recorded signal already mentioned but also shows a reduction of the membrane potential as compared to the applied voltage. As these effects both rely on $R_{\text {series }}$, they are commonly referred to as 'series resistance errors'. These effects are especially problematic for recordings from large cells with low membrane resistances such as Xenopus oocytes. In such cells, fast processes such as the gating of $\mathrm{Na}^{+}$ channels cannot be properly resolved in time even when using two electrode voltage clamp with electrode resistances below $1 \mathrm{M} \Omega$. For small cells with large membrane resistances compared to the input resistance, $\tau$ is reduced to $\tau=C R_{\text {series }}$ and the membrane potential comes close to $V_{c}$ (Usually with an error in the order of $R_{\text {series }}(R)$. The above only deals with the response to changing electrode voltage though. To show the non-instantaneous reaction to a changing current due to changes in the membrane resistance $R$ we keep $V_{c}$ constant. The derivative of the right side of equation 2.2 .5 then becomes

$$
I_{\text {injected }}=\frac{V_{c}-V_{m}}{R_{\text {series }}} \Rightarrow \frac{d V_{m}}{d t}=-R_{\text {series }} \frac{d I_{\text {injected }}}{d t} .
$$

By inserting this result into the left side of equation 2.2.5 we obtain

$$
I_{\text {Ohm }}=I_{\text {injected }}+C R_{\text {series }} \frac{d I_{\text {injected }}}{d t} .
$$

As can be seen, the injected current does not follow the membrane current instantaneously, and the time constant needed for the approach is once again $\tau=C R_{\text {series }}$. It should also be noted, that although the injected current does reach $I_{O h m}$ in this case, equation 2.2.7 still states that the membrane voltage does not perfectly represent the voltage at the pipette. This means, that $I_{O h m}$ does not represent the exact current at $V_{c}$ but $I_{O h m}=\left(V_{m}-V_{r}\right) / R \neq\left(V_{c}-V_{r}\right) / R$. 


\subsection{The patch-clamp method}

To characterize the properties of ion channels embedded into a cell membrane, delicate measurements of the currents through these channels need to be performed. The most common strategy to date is 'patch clamp', a technique evolved from voltage clamp in which one electrode is placed on each side of the cell membrane, in order to determine the current through (or the voltage drop across) that membrane. The term 'patch clamp' derives from the fact, that the recording electrode is situated in a glass pipette, restricting the path of the current to the pipette opening. By pressing the pipette tip onto the membrane of a cell, ion flow can only take place through the patch covered by the opening. Doing so leads to the most basic configuration of patch clamp the 'cell attached' mode putting the cell in between the recording electrode inside the pipette and a reference electrode in the bath solution outside the cell. Pressing the pipette onto the cell's lipid membrane (or more commonly lifting the membrane to the pipette by negative pressure), leads to attachment of the membrane to the clean glass surface by electrostatic interactions and the formation of a tight seal with resistances of more than $1 \mathrm{G} \Omega$, commonly referred to simply as 'gigaseal'. This procedure insures that almost all current between recording- and reference electrode passes through ion channels, as the gigaseal prohibits large stray currents. For channels with high enough conductivity, single ion channel currents can be isolated in this configuration [2]. The low leak-currents also improve the signal to noise ratio. This is due to the fact that the leak conductance $G_{\text {leak }}=1 / R_{\text {leak }}$ enables a pathway for membrane current $I_{m}$ to close the circle without passing through the recording electrode. The recorded current is thus reduced to

$$
I_{p}=\frac{I_{m} R_{\text {leak }}}{R_{\text {leak }}+R_{\text {pipette }}}
$$

The Johnson noise in the voltage is given by $\left\langle V^{2}\right\rangle=4 k_{B} T R f_{c}$ ([13] pp. $\left.150-160\right)$ with $k_{B}$ being the Boltzmann constant, $T$ the temperature, $R$ the resistance and $f_{c}$ the cutoff frequency of the measurement. As $I=V / R$ and $R=R_{\text {leak }}+R_{\text {pipette }}$ in our case, the current noise becomes $\left\langle I^{2}\right\rangle=4 k_{B} T f_{c} /\left(R_{\text {leak }}+R_{\text {pipette }}\right)$ and the signal to noise ratio becomes thus

$$
S / N=\frac{I_{m} R_{\text {leak }}}{4 k_{B} T f_{c}}
$$

To measure the voltage inside the cell or the currents through the entire membrane instead of single channels, the recording electrode needs to be in electric contact with the cell's interior. This is possible by physically inserting the electrodes into the cell. For most cells, this requires small electrodes, for example thin glass pipettes [14, 15, 16]. A negative consequence is that small electrodes possess large resistances, limiting the possible current injection. However, by removing the membrane of a fairly large patch (usually pipette diameters of around $1 \mu \mathrm{m}$ are used) in the cell attached configuration, the resistance between the recording electrode and the cells interior becomes so small (usually no more than three times the pipette resistance 
in free solution) that the voltage of the cell can be clamped better than with an intracellular single electrode. To rip the membrane, a short pressure pulse often accompanied by a short voltage pulse is applied. The resulting configuration is called 'whole cell' and is generally used for smaller cells, which are easier to keep at a relative homogeneously clamped voltage even with a single pipette. Due to the large pipette volume compared to the cell dimensions, a dilution of the internal cell solution takes place in the whole-cell configuration, replacing the natural ion concentrations with those of the pipette solution. Being able to control both, outer and inner ion concentrations not only decreases variability between experiments, but also enables determination of the exact influence of certain ion types. During cell attached measurements, the internal solution cannot be exchanged in such a way. However, a patch of membrane can be separated from the cell by moving the pipette away from the cell after giga-seal formation. Doing so immediately after cell attachment or in the whole-cell configuration leads to different variants of this strategy. In the later case, an 'outside-out' patch will form with the outer lipid layer of the cell facing the bath solution. If the patch is pulled from the cell in the cell-attached mode instead, the removed membrane will be in an 'inside-out' configuration. The same basic experiments can be performed using outside-out and inside-out configurations, but as it is easier to change the bath solution than that of the pipette while investigating a membrane patch, the configuration is chosen according to the desired measurement protocols. A visual representation of the different patch clamp configurations is given in Figure 2.2.

While the ability to observe single-channel opening/closing events as steps in the current waveform is a large advantage of recording from an excised patch as compared to whole-cell, other advantages stemming from the inherent errors of patch-clamp recordings are also important to note. As thoroughly explained in section 2.2 , patchclamp recordings suffer low-pass filtering with a time constant of $\tau \approx C_{\text {total }} R_{\text {series }}$. Reducing either the series resistance or the capacitance thus makes it possible to measure faster changes in the current. As the membrane patch in inside-out/outsideout recordings is usually only several square micrometers in size, the inherent capacitance is far smaller than in whole-cell recordings and the low-pass filtering is greatly reduced. Patches usually possess capacitances of between $0.01 \mathrm{pF}$ and $0.25 \mathrm{pF}$ ([17] pp. 37-51) which is about two orders of magnitude below whole-cell capacitances. In this situation the stray capacitances forming between conducting surfaces in the setup (such as the capacitor forming at the pipette wall) contribute a large part of the total capacitance. However, these can be effectively minimized so that no noticeable filtering below several thousand Hertz takes place. 


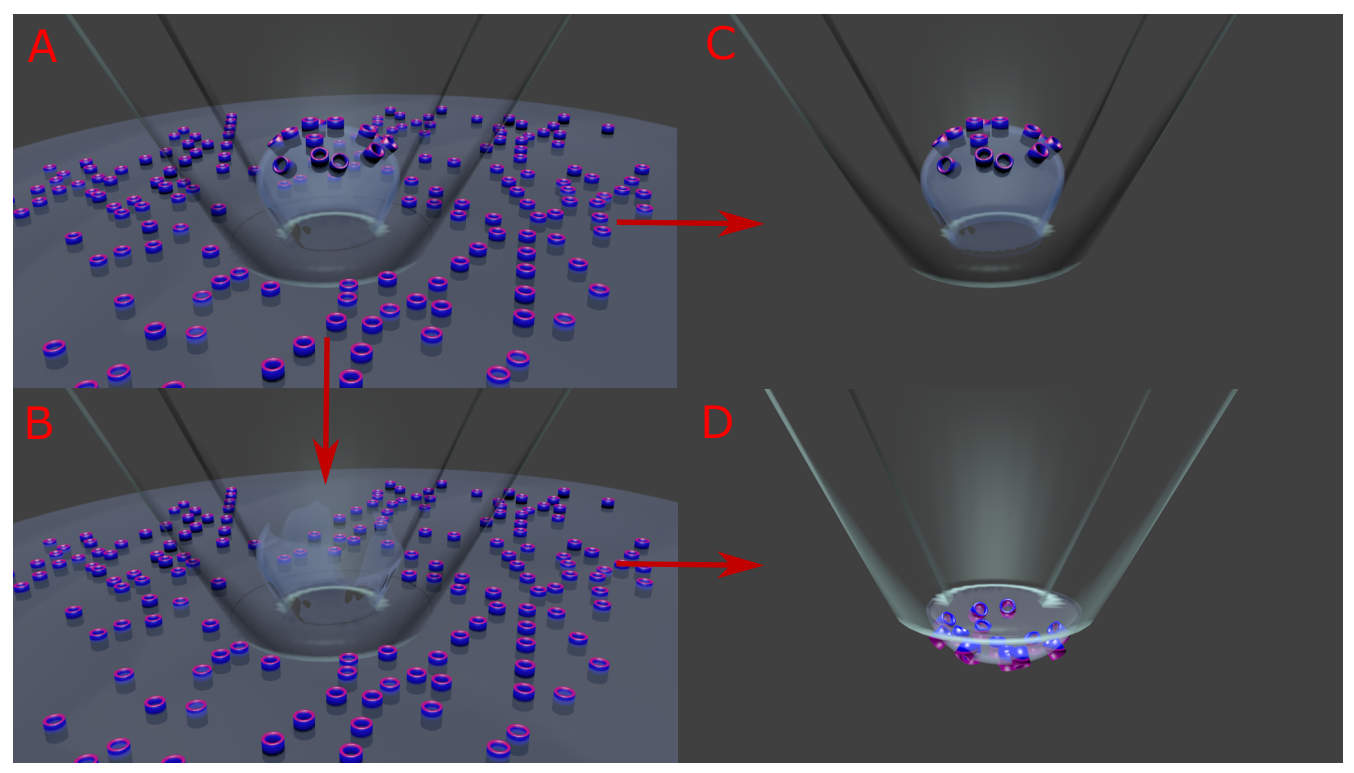

Figure 2.2: Configurations of the patch-clamp technique. Starting from the cellattached mode (A), breaking the membrane of the patch leads to the whole-cell configuration (B), giving low resistance access to the cell interior. The inside-out configuration $(\mathbf{C})$ is reached by pulling the pipette away from the cell in cell-attached mode while the outside-out configuration (D) can be achieved by pulling it away while in the whole-cell configuration. Blue cylinders represent ion channels and the magenta coloring indicates the protein side facing the outside of the cell. 


\subsection{The patch-clamp amplifier}

When dealing with such delicate systems as living cells, small current injections in the form of ion fluxes have large consequences.

As mentioned in section 2.2, the capacitance of a lipid bilayer membrane is $C_{s} \approx$ $1 \mu \mathrm{F} / \mathrm{cm}^{2}$. For cylindrical objects such as idealized axon segments the area of surrounding membrane is $A=2 \pi r l$. This means that the amount of charge $Q$ needed to achieve a depolarization $\Delta V$ is

$$
Q=C_{s} A \Delta V=2 \pi r l C_{s} \Delta V
$$

with $r$ and $l$ being the radius and length of the cylinder segment respectively. The initial part of the axon before myelination is about $50 \mu \mathrm{m}$ in layer 5 pyramidal neurons for example [18]. A $50 \mu \mathrm{m}$ long segment with $1 \mu \mathrm{m}$ diameter means $A \approx$ $1.6 \times 10^{-6} \mathrm{~cm}^{2}$ and

$$
Q \approx 1.6 \times 10^{-15} \frac{C}{m V} \Delta V \approx 1 \times 10^{4} \frac{e}{m V} \Delta V
$$

with $e$ being the elementary charge. This means it only takes 10000 ions or $1.6 \mathrm{fA}$ to depolarize the AIS by $1 \mathrm{mV}$ in $1 \mathrm{~s}$ under the assumption that no regulatory charge transfer takes place.

For this reason, recording equipment is required that does not load down the source (cell) and draws only minimal current from it. Modern patch-clamp amplifiers employ unity-gain buffer amplifiers based on operational amplifiers (op-amps) in a negative feedback configuration as seen in Figure 2.3. The input impedance of operational amplifiers is very high (up to $10 \mathrm{~T} \Omega$ ) while the output impedance is very low. Due to the high input impedance the requirement of drawing little current is satisfied and thanks to the low output impedance it drives the load as if it were a perfect voltage source.

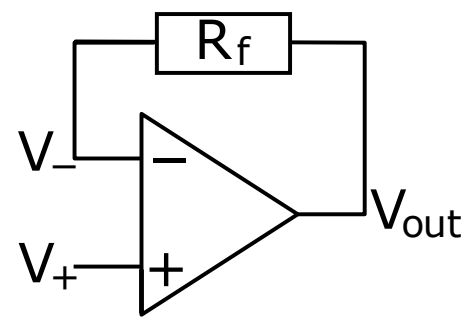

Figure 2.3: Operational amplifier with negative feedback. The negative feedback on an operational amplifier $\overline{\text { leads }}$ to an equal potential at both inputs. Due to the high input resistance of op-amps, the circuit draws nearly no current from the inputs.

By feeding the output $V_{\text {out }}=A\left(V_{+}-V_{-}\right)$(gain factor A) back into the negative input the relation

$$
V_{+}=V_{-}
$$

is closely approached with $V_{+}$and $V_{-}$being the potential at the positive and negative input respectively. With such a negative feedback loop it is possible to create both a current follower (voltage clamp) and a voltage follower (current clamp) by either connecting the recording electrode to the negative or the positive input as presented in Figure 2.4. That the output voltage equals the electrode voltage is obvious in the 
voltage follower. Due to equation 2.4.2 and the fact that no current is drawn by the amplifier, the output voltage is

$$
V_{\text {out }}=V_{c}-I_{p} R_{f}
$$

for the current follower, mirroring the current through the electrode $I_{p}$. Figure 2.4 already incorporates a parasitic capacitance $C_{p}$ (created to a large degree by the conductor forming at the pipette wall). Without the compensation circuit shown in red, this leads to errors in the measurement, as this capacitance needs to be charged or discharged by the current through the electrode. In both current and voltage follower the general strategy of neutralizing this effect is the same: A second capacitance is introduced discharging and charging in a manner opposed to the unwanted capacitance so that this charging current is not present at the amplifier output. An example for the simpler case of capacitance neutralization for the voltage follower will make the process clearer: If the neutralization gain is $A_{n}=2$ and the capacitance $C_{n}=C_{p}$ then the voltage drop at $C_{n}$ is $2 V_{\text {out }}-V_{e}=V_{e}$ opposing that at $C_{p}$. All the current to $C_{p}$ thus comes from $C_{n}$ if $v_{e}$ changes. It should be noted that even though just one neutralization capacitor is shown in Figure 2.4, an additional parallel branch exists to compensate for the charging of the cell capacitance discussed in section 2.2.

Section 2.2 also explains the errors due to the series resistance $R_{\text {series }}$ (i.e. the resistance of the electrode). These errors can also be reduced electronically by a positive feedback circuit as shown in Figure 2.4 (C). To understand the workings of this circuit it is helpful to examine the series resistance error from a slightly different angle. When clamping the electrode to a certain control voltage $V_{c}$, the series resistance acts as a voltage divider, and the actual membrane voltage $V_{m}$ is reduced by the voltage drop across the series resistance $V_{\text {error }}=R_{\text {series }} I_{p}$. The series resistance error is thus proportional to the current provided by the circuit. By increasing the control voltage $V_{c}$ by a value proportional to $I_{p}$, the current flow into the cell increases just as it would for an electrode with a reduced resistance. As $R_{\text {series }}$ can be estimated quite well from the neutralization of the cell capacitance shown above, it would in theory be possible to simulate a pipette with zero series resistance. However, as the positive feedback would become unstable, oscillations in the voltage become increasingly strong as $100 \%$ compensation is approached. This is why in practice compensation in the course of this thesis was usually kept between 40 and $70 \%$. 

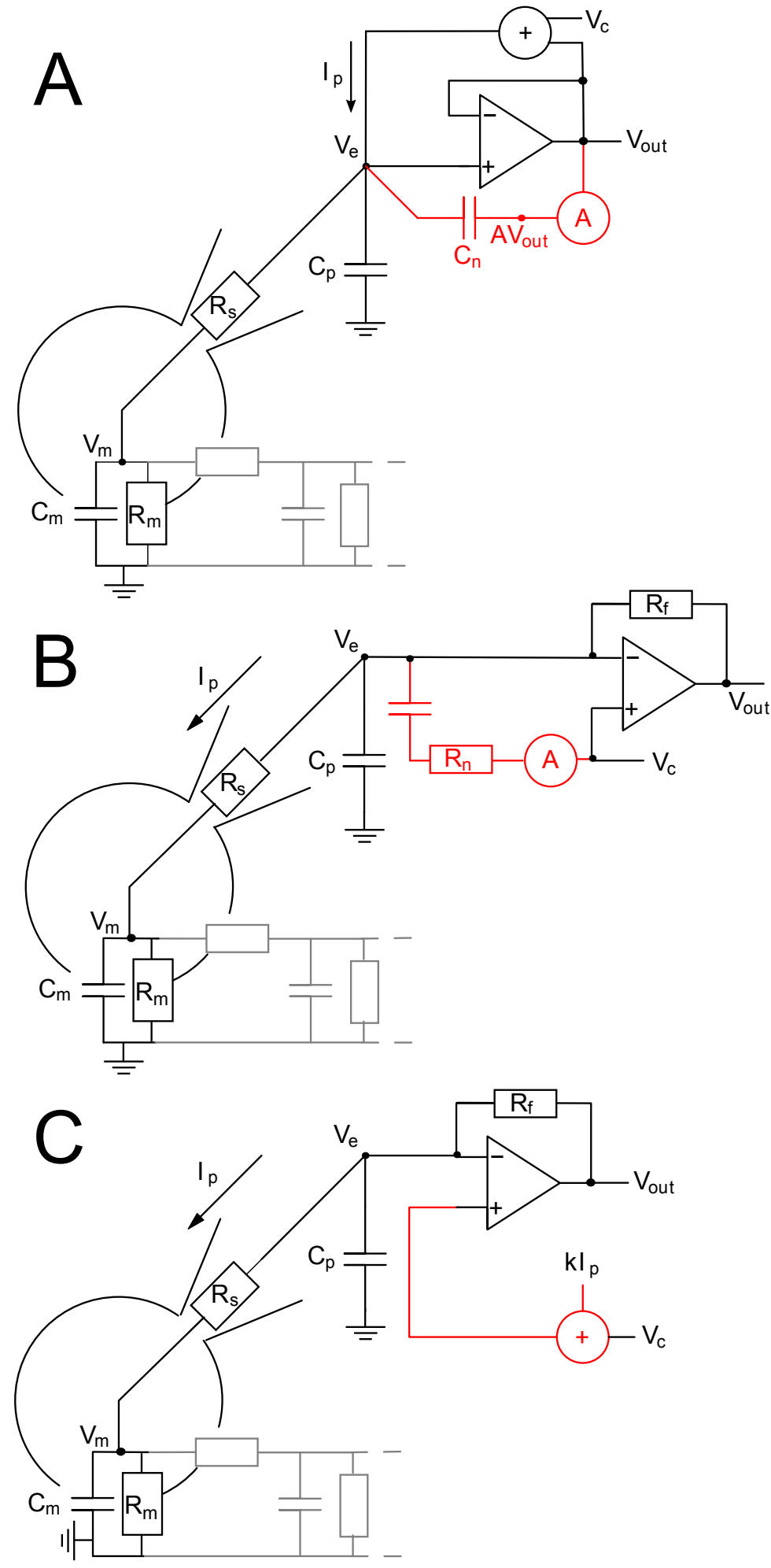

Figure 2.4: Patch-clamp amplifier circuits. A: The current-clamp amplifier consists of a high quality current source (usually another op-amp driven with the summed voltage of $V_{\text {out }}$ and a control voltage) injecting the current $I_{p}$ through the pipette resistance $R_{s}$ into the cell. The resulting potential $V_{e}$ is mirrored at $V_{\text {out }}$ due to the negative feedback. Red lines indicate the capacitance neutralization. B: The voltage-clamp amplifier keeps $V_{e}$ at a controlled value using negative feedback. $I_{p}$ can be derived from $V_{\text {out }}$ through equation 2.4.3 C: The series resistance error can be partially compensated by the circuit shown in red. By implementing a current dependent increase of the control voltage (and thus $\left.V_{e}\right)$, the current passed through $R_{s}$ will be equal to that of an electrode with lower resistance at constant $V_{c}$. Gray lines indicate the existence of additional compartments. Circuits adapted from [19]. 


\section{Scanning Ion Conductance Microscopy (SICM)}

\subsection{The principle of SICM}

Historically, experimenters have chosen configurations in which the recording electrode is placed inside a glass pipette for two major reasons: First, small tips in the micrometer range and below could be formed to penetrate the cell membrane with little injury. Second, it enabled the usage of reversible, non polarizing $\mathrm{Ag} / \mathrm{AgCl}$ electrodes [14, 15]. Filling the pipette with highly concentrated ionic solution (that for small tip openings and highly viscous solutions remain mainly inside the pipette) reduced the resistance of pipette tips. As the solution surrounding the electrode positioned at the end of the pipette tip does not change drastically during an experiment the junction potential also stays constant. Although glass microelectrodes are still used for intracellular recordings to this day [16], the idea of utilizing the (still not perfectly understood) formation of gigaseals in the patch-clamp configuration which enabled Neher and Sackmann to record single channel currents has become the most important aspect of the glass pipette [20, 2, 21] . In a similar, though not quite as groundbreaking fashion, Hansma et al. in 1989 found a way to utilize another property of the glass pipette configuration which before found only little attention: The access resistance increase of a patch pipette approaching a surface [22]. This effect finds common usage in positioning patch pipettes close enough to the membrane to start the procedure of forming a seal. It is based on the fact, that the wall of the pipette (especially for relatively thick walled pipettes) and any surrounding surfaces limit the path ions can take to reach the pipette opening. (see Figure 3.1

The resistance of a given ionic solution increases as the available area perpendicular to the ion flow decreases. This leads to a higher resistance at closer proximity to a surface. In the case of a perfectly flat pipette tip and surface, the access to the pipette opening is limited to a cylinder with a height equal to the pipette tip-tosurface distance and a radius equal to that of the outer pipette wall. For very close proximity to the surface, the access resistance is well approximated by that of the shell of the cylinder extending from the outer to the inner wall of the pipette as indicated in blue in Figure 3.1 (A). The resistance of this shell $R_{\text {shell }}$ can be calculated in the following way:

$$
R_{\text {shell }}=\int_{r_{\text {in }}}^{r_{\text {out }}} \frac{\rho d x}{A_{\text {cylinder }}(x)}=\int_{r_{\text {in }}}^{r_{\text {out }}} \frac{\rho}{2 \pi h x} d x=\frac{\rho}{2 \pi h} \ln \left(r_{\text {out }} / r_{\text {in }}\right),
$$

where $\rho$ is the specific resistance of the solution, $h$ the tip-to-surface distance and 

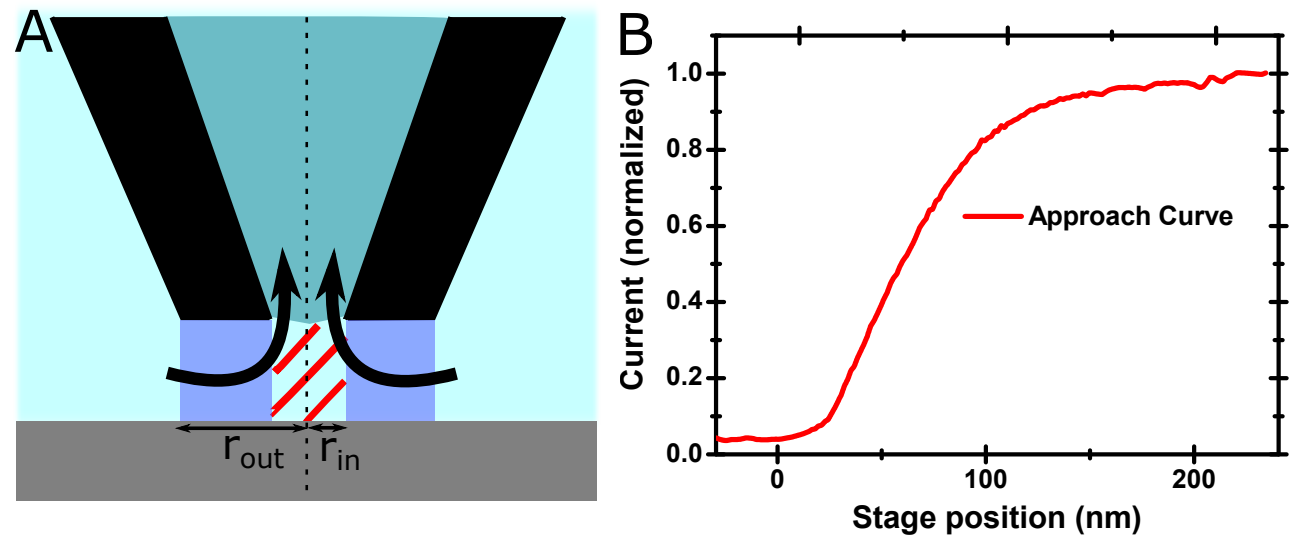

Figure 3.1: Access resistance at a surface. A: A perfectly flat pipette tip close to a perfectly flat surface. The access of ions (indicated by black arrows) to the pipette opening is limited by the surrounding surfaces. For very small tip-to-sample distances, the resistance is well approximated by the hollow cylinder formed by the medium between the outer and inner wall of the pipette (shown in blue) as horizontal current flow dominates the resistance. For larger distances the inner cylinder indicated by red stripes also becomes relevant. B: Experimentally measured relationship between current and tip-to-sample distance.

$r_{i n}$ and $r_{\text {out }}$ are the radii of the inner and outer wall of the pipette tip respectively. For slightly larger tip-to-surface distances the effect of the resistance of the inner cylinder opposing the opening of the tip must be taken into account. Nitz et al. approximated this as the sum of a resistance for purely horizontal current passing from the inner pipette wall to half the distance to the center of the opening and a purely vertical current traversing half the cylinder height [23]. These two resistances add up to

$$
R_{\text {innercylinder }}=\frac{\rho}{2 \pi h} \ln \left(r_{i n} / 0.5 r_{i n}\right)+\frac{\rho h}{\pi r_{i n}^{2}} .
$$

The entire resistance would thus be

$$
R_{\text {total }}=\frac{\rho}{2 \pi h}\left(\ln (2)+\ln \left(r_{\text {out }} / r_{\text {in }}\right)\right)+\frac{\rho h}{\pi r_{\text {in }}^{2}} .
$$

It should be noted, that this commonly cited approximation [24, 25, 26] only holds for close distances and leads to an infinitely high resistance for infinite $h$. It also does not take the non-homogeneous electric field at the tip into account. In reality the access resistance reaches a finite value for infinite distances as explained in section 6.1.1.

By applying a constant voltage, the access resistance increase is directly measurable by the current decrease through the pipette as shown in Figure 3.1 (B). Using the rising access resistance as the pipette comes closer to a surface as a measure of distance, the heights of a sample can be determined at any point similar to other techniques such as atomic force microscopy (AFM) or scanning tunneling 
microscopy [27, 28]. The general method relies on precise motors such as piezo stages to move the probe (in this case the pipette) in all spatial dimensions. In the height direction the feedback signal (the access resistance) is used so that the probe-to-sample distance never decreases to less than a certain threshold value. By recording the motor position at which the threshold is reached, the relative height at every position of the sample can be determined. Topographic images are created by raster scanning the sample and plotting the height of the sample over the positions of the scan. (see section 6.2.1 for a more detailed description) The resulting method is termed 'scanning ion conductance microscopy' (SICM) and possesses some unique and interesting properties.

\section{Characteristics of SICM}

As the feedback variable (current/resistance) is nearly entirely dependent on the physical obstruction of the ion flow, the interaction of the pipette tip and the measured sample is nearly non existent. This means, that in contrast to AFM measurements no force is exerted onto the sample and less mechanical deformation of very soft samples takes place [29, 30]. This is ideal for fragile biological systems such as membranes or small cell filaments such as dendrite trees in neurons. Figure 3.2 shows an example of images recorded via AFM and SICM. The structure seen is the thin bridge connecting two daughter cells just before the final stage of cell division. In living cells this bridge is not anchored to the substrate as can be seen in the fluorescent image of the free moving bridge depicted in Figure 3.3. Under these conditions, an AFM tip would cause lateral displacement of the structure during the scanning procedure, making imaging impossible. For this reason the cells were treated before imaging with AFM and some of the substructures are not visible in the AFM images. Even with adhering structures such a loss of substructure information is common in AFM however, due to the deformations caused by the applied forces [29].

The lateral resolution of SICM is limited by the opening size of the pipette. While the dependence has been quantified as 3 times the inner radius [26], the exact resolution depends on the shape of the sample. This is due to the relatively complex influence of non-flat surfaces on the electric field at the pipette tip. On the one hand this makes it practically impossible to correct a recorded image in post processing by deconvolution from a smoothening function of the tip as can be done for AFM [32, 33]. On the other hand it increases the ability to resolve certain small structures as in [34], where objects only $13 \mathrm{~nm}$ apart could be clearly distinguished with pipette openings of the same size. The height resolution of SICM is far more precise than the lateral resolution and in principle is only limited by the noise in the measurement of the access resistance increase. Modern patch-clamp amplifiers offer signal-to-noise ratios that enable a vertical resolution down to the single digit nanometer regime. The pipette and amplification circuit used in SICM are readily usable for standard electrophysiological measurements. This enables a combination of high-resolution imaging of the topography with electrophysiological measurements at precise locations [35, 36]. This way the connection of topological structure and ion channels can be investigated [37, 36]. It is also possible to use the pipette for 

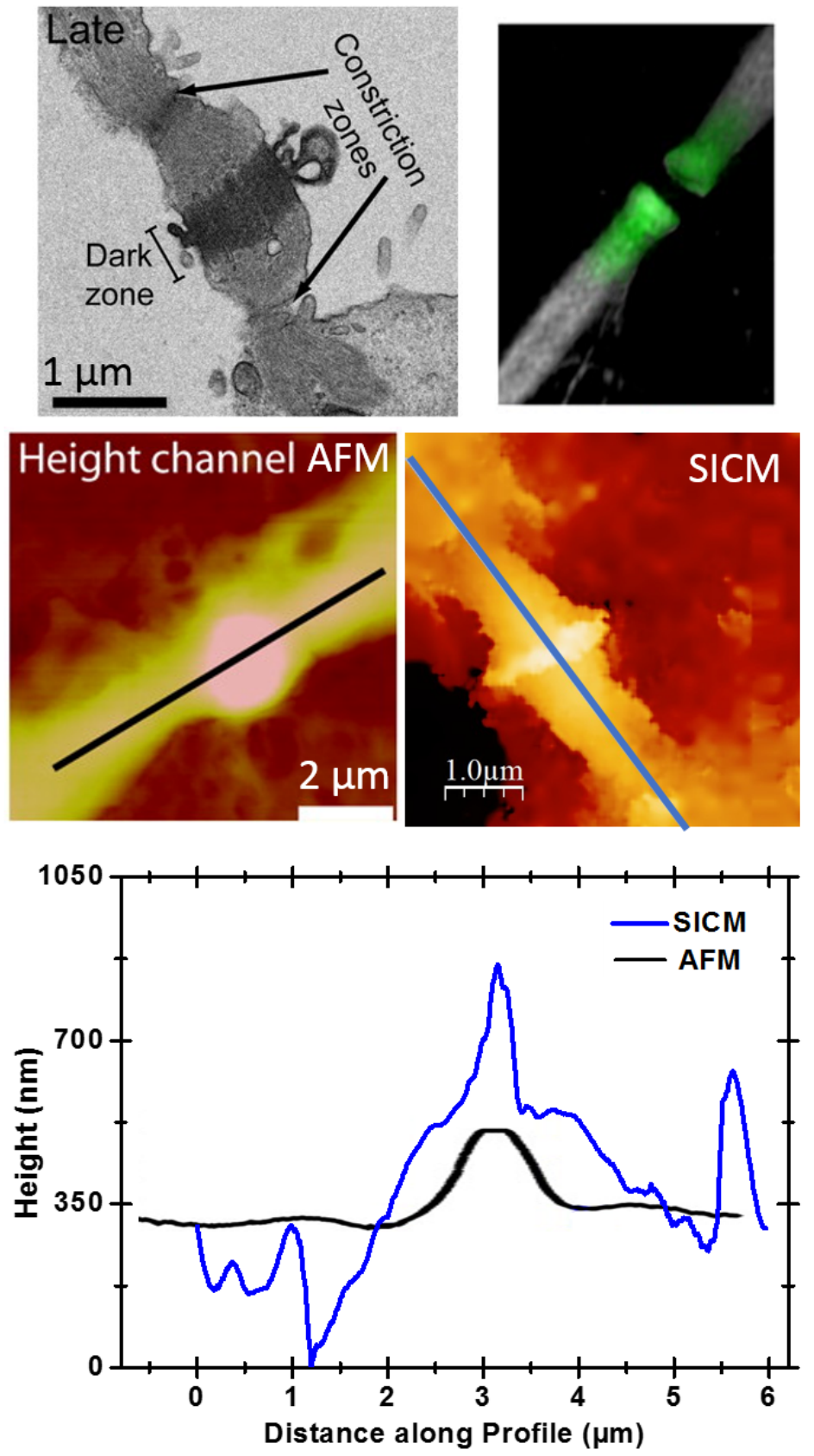

Figure 3.2: Bridge connecting two cells before abscission. Top: EM and SIM (AuroraB and $\alpha$ tubulin labeled) images of the midbody. The so called 'dark zone' is clearly visible in both images. Center: Topographic image of the bridge connecting two cells during the last stage of cell division using AFM and SICM. For AFM measurements the cells were fixed to the coverslip to prevent movement due to the contact forces, while SICM scans were performed on living free moving cells. Bottom: The height profiles along the lines drawn into the topographic images. The height of the structure is reduced for the AFM measurement and the substructure of the dark zone is lost. AFM, EM and SIM data from [31. 
the deposition of molecules at well defined locations for example to investigate the effect of drugs on very localized structures [38, 39] and to combine the SICM setup with optical methods such as fluorescence microscopy, confocal microscopy and even near-field microscopy [40, 41], enabling the integration of optical and topographical information. Recently it has been shown, that the SICM technique can even extract some mechanical properties from a sample [42, 43. This is achieved by applying hydrodynamic pressure in the pipette, which causes liquid to stream out of the pipette tip. Soft samples in close proximity to the pipette opening are deformed by the force of the liquid stream. By comparing the reduction of electric currents when approaching a sample from above with and without applied pressure, information about the mechanical properties of the sample can be inferred. 


\subsection{Investigation of the abscission process during cell division}

Topographical imaging using SICM can easily be performed in physiological solutions, making it ideally suited for imaging living cells. The strengths of SICM when compared to AFM become clearly visible when investigating the process of 'abscission', a process taking place at the final stage of cell division.

We collaborated with Natalie Elia (Ben-Gurion University, Beer-Sheva, Israel) who has performed investigations of this event using a broad range of techniques. Some examples are shown in Figure 3.2 .

Abscission takes place at a time in the cell cycle, when the mother cell has already divided itself into two independently functioning daughter cells, and the membrane has constricted in between them leaving only a thin 'bridge' with a diameter of $1-2 \mu \mathrm{m}$ [44].

During abscission this last bridge connecting the two daughter cells after cytokinesis is severed. Numerous microtubules take part in cell division, most notably during the separation of chromosomes during mitosis. During the contraction of the cell membrane dividing the cell, many of these microtubules are present in two anti-parallel arrays in the connecting bridge with plus ends interlocking [45]. This means, that before the final connection can be severed, the microtubules have to be disassembled. This is most likely performed by spastin [46] a microtubule-severing protein mediated by the 'endosomal sorting complexes required for transport' (ESCRT) also involved in the final membrane abscission. Cutting of the bridge takes place at two locations, leaving not only the daughter cells, but also a small part of the bridge called the 'midbody', a tightly packed structure of about $2 \mu \mathrm{m}$ length which is already discernible before final abscission by fluorescent imaging as seen in Figure 3.3. Under physiological conditions the whole process only requires about 20 minutes for Madin-Darby canine kidney (MDCK) cells [31]. As it is usually not attached to the surface, determining a high resolution topography of the bridge via AFM is difficult on living cells. Fixation of cells on the other hand can lead to a change in the morphology. Figure 3.3 (A) shows a fluorescent image of tubulin labeled MDCK cells imaged at room temperature at three different stages of abscission. The movement of the connecting bridge is clearly visible, especially after the first cutting event $(\mathrm{t}=138 \mathrm{~min})$. Figure 3.3 (B) shows the average speed of movement as defined by the displacement between each frame. This speed limits the time of acquisition available for an image when using living cells.

SICM can however determine the topography with high precision only if the movement of the cell is slow enough not to disturb the imaging. Results for such measurements along with an AFM comparison on fixed structures are shown in Figure 3.2. This measurement was performed at $21^{\circ} \mathrm{C}$ on modified MDCK cells with a GFPtagged $\alpha$-tubulin for optical identification of the midbodies. Due to the slowed division kinetics, changes to the morphology were small during the 5 minutes required to image the bridge. Figure 3.4 (A-C) shows the profiles along the midbody and bridge for three cells as they change over time. Figure 3.4 (A) presents a cell in an early stage of abscission in which the dark zone exhibits no prominent topographic 

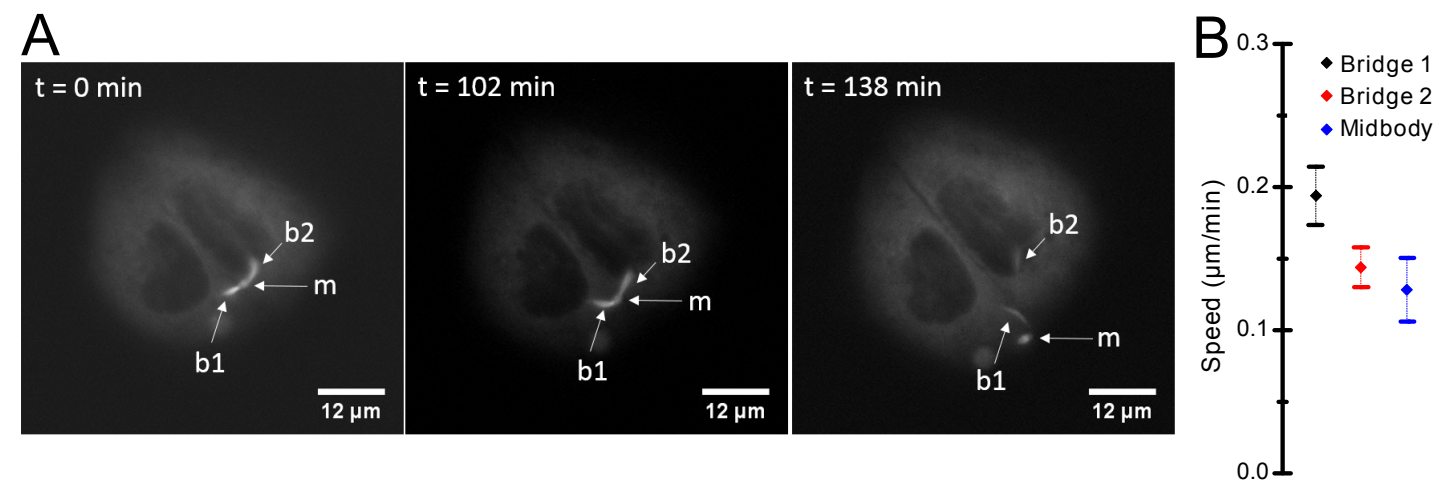

Figure 3.3: Fluorescent microscopy of midbody. A: Fluorescent image of $\alpha$-tubulin labeled MDCK cells imaged at room temperature at three different times. Arrows point to the positions of the midbody $(\mathrm{m})$ and the tubulin bundles of the bridge (b1 \& b2). B: Speed of the three structures indicated in (A) as defined by the displacement between frames divided by the inter frame time $(3 \mathrm{~min})$. Only the frames before the cutting event are considered $(n=28)$. The diamonds represent the mean and the horizontal lines the standard error of the mean.

structure, while Figure 3.4 (B) displays a cell in which this structure is gradually forming. Figure 3.4 (C) shows the profiles during a time series spanning more than 12 hours, which made it possible to examine the development from the early stages until the cutting occurred. This data is examined further in Figure 3.4 (D-F) by extracting the increase in height and width of the central structure of the midbody. In this dense, small structure, transport and arrangement of all the necessary components is not trivial. Evidence suggests that the abscission complex arranges in an asymmetric way to first cleave one side of the bridge [44]. The asymmetry of the structure can be seen clearly in the topographic structure of Figure 3.4 (D-F) showing that at least some of the structures involved in abscission are visible in the topography of the membrane.

\section{Future endeavors}

The ability to track the internal processes during abscission by topographical imaging is highly dependent on the resolution of the image. In [34] single proteins were identified and structures only $13 \mathrm{~nm}$ apart were distinguished. While achieving such a resolution in a living, dividing cell seems unlikely, a substantial increase is still possible. The main problem faced is the small timespan available for image acquisition due to the movement of the structure as shown in Figure 3.3. By slowing the metabolism of the cell even more, the resolution of the image could thus be increased. Another method for faster data acquisition is limiting the structure scanned. For our original SICM setup the possibilities to do so are extremely limited as we have no access to the source code of the recording software. However, by using the SICM prototype described in section 6.1.2, I have managed to reduce the measurement of the topography to high resolution line scans, effectively only recording the profiles along the midbody. Figure 3.5 shows an example of such a scan. The recording of 

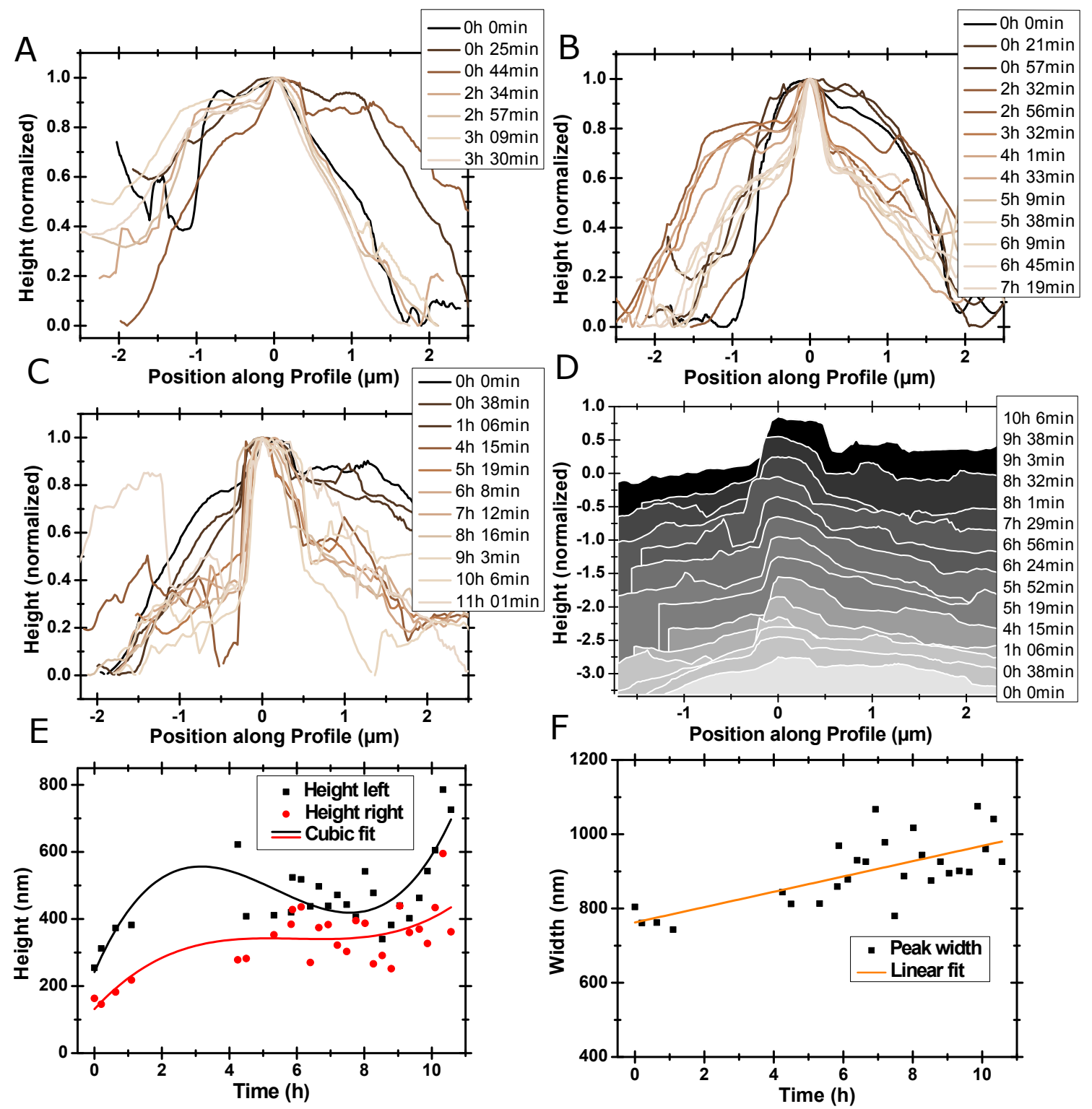

Figure 3.4: Midbody profiles. A-C: Normalized profiles along the midbody in three different cells. A: Cell at an early stage just before the dark zone rises. B: Cell during rise of dark zone. C: Cell imaged during entire abscission process. D: Same cell as $\mathbf{C}$ with shifted heights so that morphological changes in the dark zone can be seen more easily. E: Height of the dark zone on left and right side over time. An asymmetric height increase of the dark zone can be observed. Lines show a third order polynomial fit. F: Width increase of dark zone over time. 
the line scan only took about one eighth of the time it took to record a full image at the same resolution. This time can be used to increase the lateral resolution as was done in this case as well as the vertical resolution. The latter is possible by increasing the setpoint during scanning (which reduces the distance between the probe and the sample). When using high setpoints the approach of the sample must be slowed to avoid accidental collisions however, which increases the scanning time. (See section 6.2.1 for more details about scanning times.)

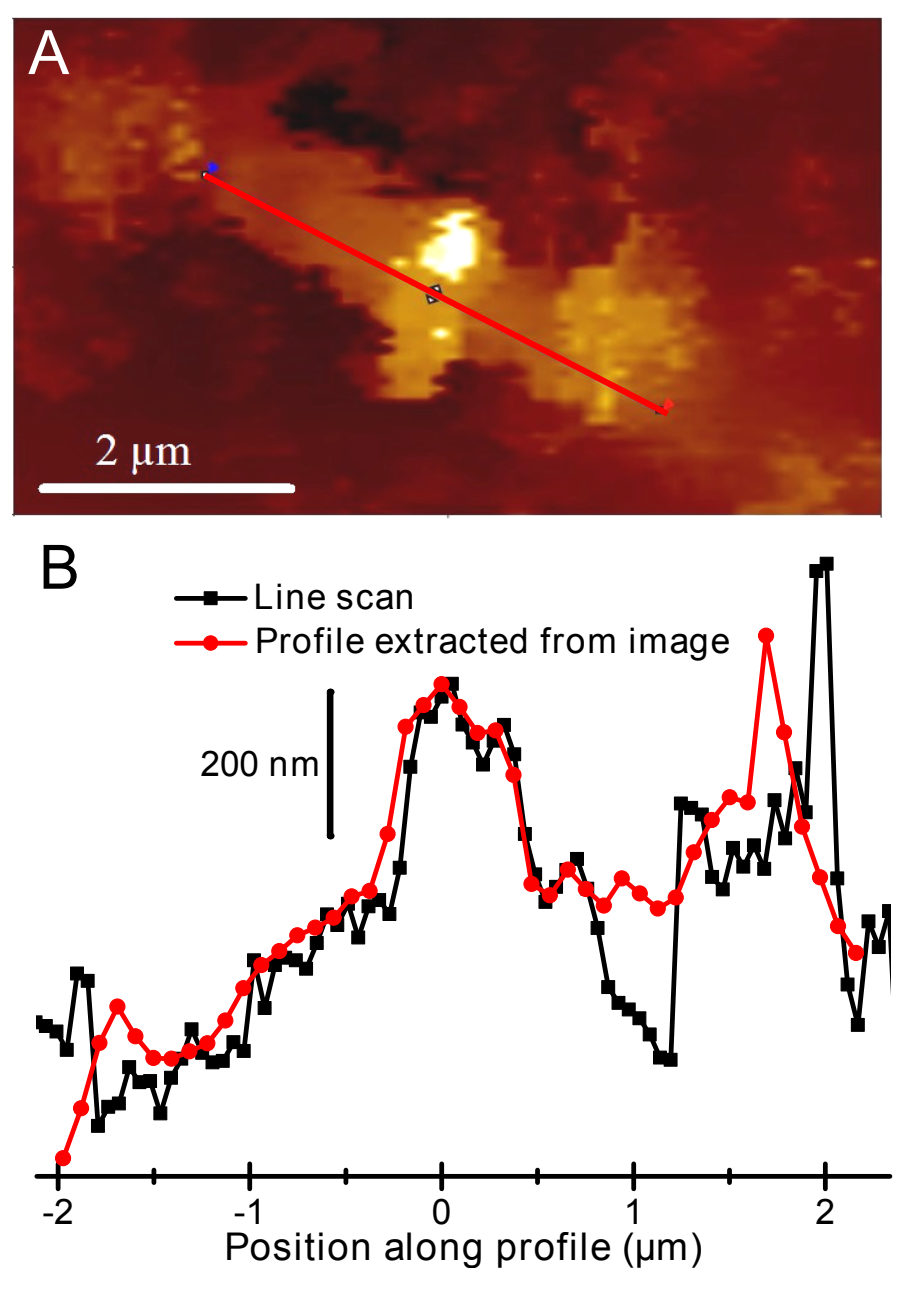

Figure 3.5: Line scanning of midbody. A: Image of the midbody structure recorded in the prototype setup. The image quality appears generally inferior to that of the old setup seen in Figure 3.2. This is due to the fact, that the prototype performs only evenly spaced line scans, while the old setup has a highly adaptive scanning protocol (see section 6.2.1). The latter yields larger local resolutions at the structures of interest. B: Height profiles of the midbody. The red curve is extracted from the image in A while the black curve is a line scan across the structure. The lateral resolution of the line scan is about twice that of the complete image while the recording time was only about one fourth. The vertical resolution was not increased in this case.

As explained above, the cell activity should be as low as possible while performing scans. This slows down the time of the entire abscission process however, so that an investigation of the topographical changes during the entire process can last 12 hours as seen in Figure 3.4. This is problematic, as the scanning position needs to be adjusted to the moving structure. This adjustment can only be performed manually so far, implying supervision by the experimenter for the entire measurement. Additional problems arise from the high chance of contamination of the pipette tip during such a long measurement. Exchanging the probe for a new pipette is possible in such cases, but requires considerable time and increases the chance of losing track of the investigated structure. In order to increase the cell activity and 
thereby decrease the required recording time, I designed a heatable holder for the new SICM prototype. It consists of a thermally isolated brass metal ring heated by a resistive heater. The petri dish is placed into the ring and the temperature of the metal is measured using a thermistor for feedback. Control measurements on AFM calibration standards heated to physiological temperatures have shown that the system is able to perform scans at these temperatures. As expected, the cell activity at physiological temperatures is too high for imaging without blurring. However, due to the relatively fast cooling of the metal ring it is possible to perform heating phases followed by scanning phases to provide slow moving cells for scanning while reducing the overall time of cell division. 


\section{Electrophysiology/ SICM in neuroscience}

While electrophysiological measurements find usage in a large number of fields, including the investigation of muscle cells [36, 35, 47], plants [48, 49] and bacteria [50, 51], the most prominent field of electrophysiology is neuroscience.

Since the groundbreaking discoveries of Hodgkin and Huxley [1] through the investigation of the squid giant axon, more focus has been put into investigating the much smaller cells involved in the computations of the mammal brain. Investigating these cells and the networks they build poses far greater experimental difficulties than the investigation of the giant axon. The small size of cells found in the cortex of the brain for example make an arrangement of the electrode as in the voltage clamp of Hodgkin and Huxley impossible, and the small structures require far more consideration on the influence of the recording electrode. Patch-clamp techniques are capable of performing a number of these more delicate measurements, but become highly invasive when the investigated structures are small.

This chapter presents an important effect in the signal processing of the brain and shows how this effect is highly dependent on the membrane properties of the axon initial segment, a structure of only several $10 \mu \mathrm{m}$ in length and diameters of down to less than $300 \mathrm{~nm}$. The experimental determination of the underlying structures is thus highly difficult and traditional measuring techniques have so far yielded conflicting results [10, 52, 53]. I will present a strategy to tackle this using electrophysiological measurements coupled with SICM and computer simulation. Work on the computer software needed for the simulation has been done by Andres AgudeloToro [11], neural culturing and staining was in part performed by Christopher Dilip (MPI DS, Göttingen) and neural cultures were provided by Walter Stühmer's lab (MPI Ex.Med., Göttingen). 


\subsection{Ion channels as the basis of neural activity}

Signal processing in the brain is an extremely complex process involving tens of billions of cells interacting through trillions of connections in humans [54] and has consequently not been fully understood. Breaking down the complexity by concentrating on smaller subunits is therefore a useful strategy to progress in the field.

Single neurons represent individual units of processing and transferring information. These highly specialized cells perform information processing by converting their input into digital signals which they can transfer over large distances to influence other cells.

The principle of information processing in neurons is based on the electric currents created by ions in solution. Specifically the currents passing through ion permeable proteins embedded into the highly insulating lipid membrane of the cell. It is through the use of these proteins that neurons and other cells such as cardiomyocytes create a concentration gradient of certain ions across their membrane which in turn leads to a voltage drop across said lipid bilayer. The ion permeable proteins created by the cell can be categorized into two groups fulfilling different functions. The first group of these trans-membrane proteins are the ion pumps (also called ion transporters). This group is especially important for maintaining the ion gradients across the membranes. Ion pumps are not permeable to ions in the traditional sense, as they require energy to transport ions across the cell membrane (although some ion pumps are 'leaky' meaning they are also permeable by certain ions). This active transport takes place in one direction, usually against the gradient of ion concentration. One of the most important examples is the sodium-potassium pump found in animal cells. It moves $K^{+}$ions to the outside of the cell while pumping $\mathrm{Na}^{+}$ions inside to maintain a low sodium and high potassium concentration inside the cell. The second class of proteins are ion channels. They are passive elements usually permeable to only a small selection of specific ions, meaning they offer a far lower resistance to those ions than the surrounding lipid membrane. Ions can travel through their pores in both directions and will create a net current until the electro-chemical equilibrium is reached i.e. all forces on the permeable ions sum up to zero so that no net ion flow takes place. Most ion channels possess a gating mechanism however, meaning they adapt their conductance in response to changes in their environment. This is an integral part in both the signal processing and the propagation of signals, as their gating properties enable ion channels to respond to the stimulation a cell receives. An important group of gated channels are the voltage-dependent variants. They are sensitive to the voltage difference across the membrane (or more precisely the corresponding electric field) and change their own conductance accordingly. This enables the initiation of a chain reaction leading to the formation of action potentials (AP), the digital signaling units of neural cells. These action potentials result from the concentration gradients created by ion pumps in conjuncture with the gating mechanism of the voltage sensitive channels. Non-active neurons possess a fixed ion concentration gradient at their membranes leading to the so called 'resting potential'. This defines a negative voltage across the membrane usually between $-60 \mathrm{mV}$ and $-80 \mathrm{mV}$. Small fluctuations around the 
resting potential are not able to change the conductance of voltage gated channels noticeably, making this situation relatively stable. If the potential shifts to more positive values past a certain threshold due to a large enough ionic current through the membrane, more of the voltage-dependent channels will open. This process will start with an increase in conductance for sodium, as the corresponding channels opening probability increases strongly even for small shifts to more positive potentials as shown in Figure 4.1 (C). Due to the influx of sodium into the cell its membrane potential will become even more positive, triggering three processes: Firstly, a conductance change of sodium channels in adjacent membrane patches is triggered as the threshold voltage is reached there. Secondly, the sodium channels in the region of initial sodium influx inactivate, stopping the current locally. And thirdly, potassium selective channels in close proximity to the now inactivated channels open, countering the voltage change locally. Through these processes the potential change moves along the membrane in space and time in the fashion of a wave as illustrated in Figure 4.1 This wave is the before mentioned action potential.

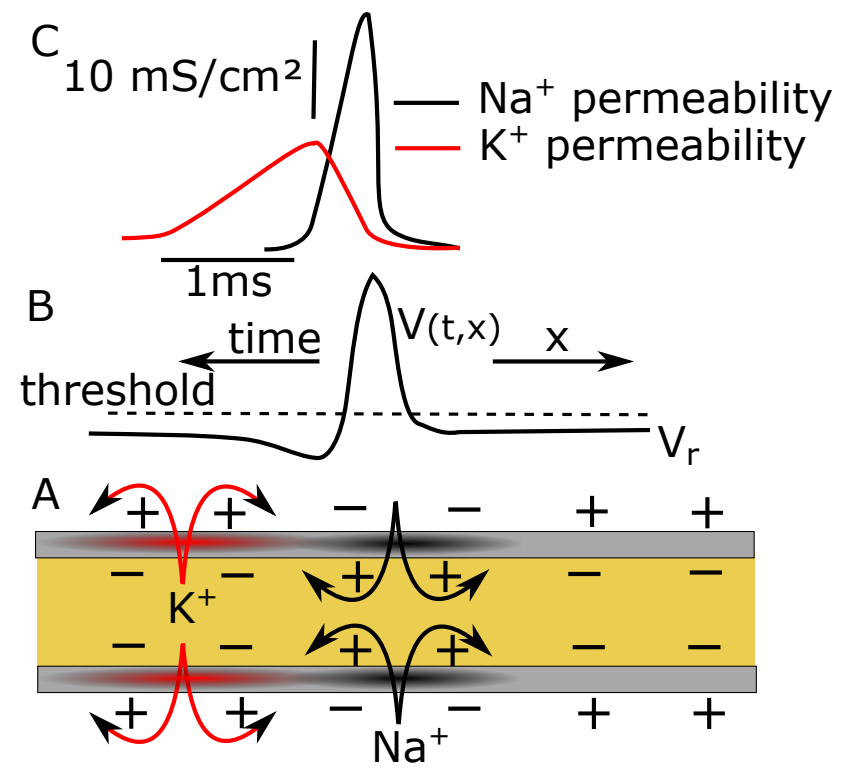

Figure 4.1: Action potential generation. Panel A shows a cylindrical axon segment at one fixed time point during AP propagation. The AP propagates from the left to the right. At the location where the threshold for $\mathrm{Na}^{+}$channel gating has just been reached the membrane conductance for sodium increases (indicated in black). A conductance increase for potassium is following the sodium influx (indicated in red). $\mathrm{B}$ : As the AP is a wave, the indicated shape can be interpreted as the voltage along the axon or the reversed time course of the voltage at one location. $\mathrm{Na}^{+}$influx causes depolarization while $K^{+}$flux leads to re-polarization (and hyper polarization) . C: The change of sodium and potassium permeability over time at one fixed position. The time axis is from right to left to be consistent with panel B.

By transversing along the axon, a thin extension of the cell reaching up to meters in length, the signal is relayed to distant cells for further processing. The signaling through APs is considered to be of a digital nature, as the exact shape of the pulse does not seem to have a large influence. Due to this fact, and the relatively stable shape of the AP, information loss in the axon does generally not take place. As the 
AP arrives at one of the synapses located at the axons endpoints and forming the connection between cells, it triggers stimulation of the next cell. Several types of synapses exist, that possess different pathways to stimulate the following cell. In the most common variants the electrical signal of the action potential is converted into a chemical signal by the release of neural transmitters. These diffuse across the 'synaptic cleft', a gap between the two cells forming the synapse. Receptors on the post-synaptic membrane react to the released transmitters and stimulate the cell through specific cascades usually resulting in ion currents influencing the membrane potential. The kind of stimulation the post-synaptic cell receives depends on the synapse type and 'strength' i.e. the amount a single AP can change the postsynaptic cell potential. At certain synapses (inhibitory synapses) arriving action potentials lead to a more negative membrane potential in the post-synaptic cell. This suppresses the formation of an action potential, while 'excitatory synapses' have the opposite effect and increase the chance of AP generation. The basic concept of information processing in neural networks is the interaction of the cell with all stimulation that it receives from its usually large number of synapses which can be on the order of thousands [55]. The output of a neuron defined by the number and timing of its action potentials, is connected to its input (the APs of all neurons connected to it on the pre-synaptic side) by the properties and positions of the synapses. Through synapse plasticity, i.e. the ability for synaptic connections to newly form or for existing ones to change their strength, the response function determining the output/input relation can be tuned to achieve a certain 'computation'. 


\subsection{Transmission speed of spike-rate coded signals}

The rate of firing, i.e. the number of APs generated per second, is believed to be an important encoding mechanism in the brain [56, 57]. The speed with which this information can be processed along a pathway through the brain is therefore limited by the time it takes for the firing rate to respond to an input from a prior computational/signaling stage. The ability of neural networks to relay such information quickly is clearly visible in many pathways. Visual-to-motion tasks like catching a suddenly appearing object are good examples. During these tasks all the necessary calculations (e.g. approximation of the speed/size of the object, evaluation of the best suited hand movement for catching) take place on timescales of less than a second, limiting the maximal time for a single transition in those pathways.

Different paradigms to model neurons or neural networks exist and put specific limitations on the speed of signal relaying.

Section 2.2 shows how the equivalent circuit connects the voltage rise to the current input. By using the mathematical relation of equation 2.2.5 the response to a current step injected into the cell (for example by a synaptic input) on the membrane voltage can be calculated. As the injected current in this consideration is a current step taking place at $t=t_{0}$, the general equation 2.2 .5 becomes

$$
\frac{V_{m}(t)-V_{r}}{R}+C \frac{d V_{m}(t)}{d t}=\Theta\left(t-t_{0}\right) I_{i n j}
$$

where $\Theta\left(t-t_{0}\right)$ denotes the Heaviside step function while $\mathrm{R}$ and $\mathrm{C}$ represent the membrane resistance and capacitance respectively.

The change to the membrane voltage $V_{m}$ is not instantaneous. Instead it approaches its final value with a time constant $\tau_{m}=R C$ termed the 'membrane time constant'. Different models used for representing neural activity yield different magnitudes to which such low-pass filtering in the neuron effects the time neural networks require to relay information. A number of neural models are based on the assumption, that the firing rate of neurons is a rectified copy of the membrane voltage [58, 59, 60]. In these models, the low-pass filtering property of the membrane directly limits the response speed of the firing rate. The cutoff frequency of a system of such neurons will be in the range of $f_{c}=\left(2 \pi \tau_{m}\right)^{-1}$ and thus usually far below $100 \mathrm{~Hz}$. The so called 'integrate and fire neurons' however show a fundamentally different behavior. These model neurons also increase their membrane voltage in response to injected current, but incorporate a reset of the membrane voltage every time the threshold $V_{\text {threshold }}$ for spike generation is reached. If such a neuron receives a current injection, its voltage increases towards a new value in the non instantaneous manner shown above. If however the membrane voltage before current injection is already close to $V_{\text {threshold }}$, and the final membrane voltage $V_{\infty}$ is thus larger than $V_{\text {threshold }}$, spike generation and membrane reset can take place in a much shorter time than $\tau_{m}$. This does not decrease the time a neuron farther along the information path requires to determine the new spike rate however. For a single neuron this rate is determined by the interval between spikes, so that a later neuron requires at least one such interval to determine the rate change. This changes drastically if large networks of such neurons are considered. If this network is not synchronized i.e. the 
distribution of membrane potentials is evenly distributed, then a certain number of cells will always be close to the spiking threshold. If a single neuron is relaying a small current step to such a large network via synaptic input, then the required time to detect a rate change no longer depends on the spike interval of a single cell, but on that of the entire population. This in turn means, that the reaction time can become infinitely fast for infinitely large networks. This behavior strongly depends on the prerequisite that the neurons are not synchronized. However, even slightly modified versions of the model such as the 'leaky integrate and fire' (LIF) neuron tend to synchronize [57]. Additionally, more biophysical neuron models predict a cutoff frequency on the order of the average firing rate or the inverse membrane time constant [61, 62, 63. While it has been shown that cells desynchronize in the presence of appropriate noise such as provided by synaptic bombardment in vivo [64 the fact that biophysical models are not able to reproduce the fast response time of LIF neurons indicates this to be an artifact of the simpler model. Surprisingly however, measurements on real neurons with low average firing rate have shown, that large networks of such cells are indeed able to provide very high cutoff frequencies [64, 65. Wei and Wolf consequently tested model neurons akin to pure LIF neurons but with a non-instantaneous spiking behavior dependent on the membrane time constant and the speed of the $\mathrm{Na}^{+}$influx [66]. The model was engineered so that artifacts due to the boundary conditions at voltage reset could be separated from the rest of the analytically derivable solution. The study indicated, that without these artifacts, the dependence of the networks cutoff frequency on the single neuron spike rate and membrane time constant disappears. Instead only the speed of the AP onset and thus the kinetics of the $\mathrm{Na}^{+}$influx into the cell limit the ability for networks to achieve fast signal transmission. 


\subsection{Action potential initialization}

As the influx of $\mathrm{Na}^{+}$current seems to play an important role in the ability to quickly respond to changing inputs, the difference between real neurons on the one hand and biophysical model neurons on the other might stem from wrong assumptions about these currents. As stated in section 4.1, the dynamics of these currents depend on the properties and the distribution of the corresponding ion channels. For biophysical models to properly reflect the behavior of real neurons a precise knowledge of these parameters is needed for the voltage gated $\mathrm{Na}^{+}$channels responsible for the initial rise of the action potential. Andreas Neef (personal communication) has performed a thorough investigation of the $\mathrm{Na}^{+}$channel properties in the soma of cortical neurons. Using these properties, and the recorded voltage shape of the action potential, the currents through the $\mathrm{Na}^{+}$channels were simulated, and the corresponding change in the voltage $(d V / d t)$ calculated. A comparison of simulated APs and real recordings at the soma in phase space $(d V / d t$ over $V(t))$ showed that the currents generated at the soma membrane are well reproduced by the simulation and that the $\mathrm{Na}^{+}$ influx mainly determines the voltage change. The initial part of the somatic action potential does not stem from currents through the cell-body membrane however. Instead it stems from currents reaching the soma from the site of AP initiation in the axon. These initial currents are reproduced far worse by the simulations as both the voltage and the membrane properties were only precisely determined in the soma. This demonstrates the need for measurements of the channel properties at the actual AP initiation site.

Determining the distribution and the gating behavior of channels in this region is challenging however. This is partly due to the fact, that the initiation of action potentials takes place at the axon initial segment (AIS) [67, 10, 53]. The AIS denotes a region of the axon between the cell body and the start of myelination and is usually several $10 \mu \mathrm{m}$ in length. In cortical neurons this structure is of extremely small dimensions with diameters of less than $300 \mathrm{~nm}$ as seen in Figure 4.3 (C). It is thus difficult to determine the properties or even the density of functional ion channels in the region. A number of different methods have been used [10, [53, 67], and the contradictory results show how easily the properties of the AIS are disturbed by traditional measurements. Figure 4.2 taken from [10] gives an example for conflicting data from different techniques. This can be attributed to several effect. One of these is the mechanical stress and deformation caused by the pipette. Another is the effect of the capacitance added to the membrane by the pipette during cell attached measurements, which can alter the electrical behavior. A non-uniform distribution of channels can also lead to wrong assumption about the general channel density from measurements on patches, and channels are believed to be anchored to the cytoskeleton which leads to fewer channels in excised patches than in the intact membrane [18]. 


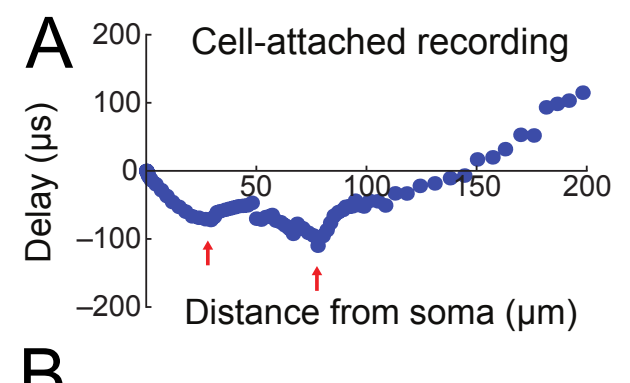

B Voltage-sensitive dye imaging
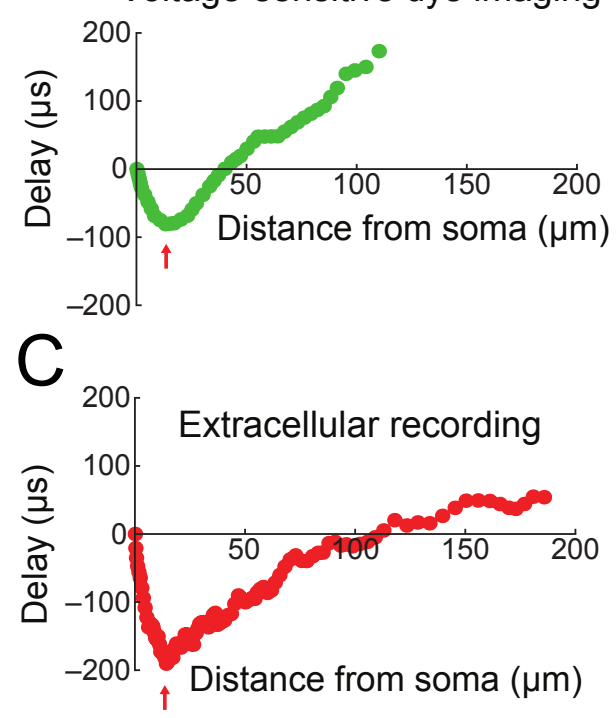

Figure 4.2: Action potential propagation in the AIS. Panel $\mathbf{A}$ shows the delay between an action potential at the soma and a certain position on the AIS, as measured via cell-attached recordings. In this case the point of AP initialization (largest negative delay) was measured far from the soma. Possibly due to the added capacitance of the pipette, shifting the site to the first node of Ranvier. B: The same effect as in A, but measured with voltage-sensitive dyes. AP initiation was measured to be at the AIS less than $100 \mu \mathrm{s}$ before the AP was registered at the soma. C: The same effect as A,B measured by extracellular recording. The initiation point is close to that of $\mathbf{B}$ but APs travel half as fast. Modified from [10].

\subsection{Electrophysiologic measurements at the axon initial segment}

Tackling the problem of determining the density and nature of ion channels in the AIS requires a technique able to measure locally without gravely disrupting the natural state of the structure. Extracellular potential recordings are capable of performing the latter, as they do not require physical contact with the cell. And unlike other methods such as voltage sensitive dyes, they possess the time resolution needed to investigate the kinetics of $\mathrm{Na}^{+}$channels. They are employed mainly in studies of large systems such as recordings in vivo or slices. These studies are usually restricted to determining if and when a neuron in a network spikes while ignoring the shape of the extracellular action potential (eAP). One of the reasons why extracellular recordings are seldom used in the investigation of ion channel densities or other sub-cellular processes is the complicated relation between signal and source as compared to patch-clamp measurements. Computational studies have shown however, that under certain conditions eAPs constrain the processes underlying AP generation better than intracellular action potentials (iAPs) i.e. the eAP shape differentiates the ion channel properties better [68]. Additionally the investigation of eAP shape gives more localized information than iAPs meaning that the influence of membrane regions neighboring the area of interest is reduced [69]. These properties make extracellular recordings a good candidate for the investigation of the AIS. The 
complicated relation between recorded signals and parameters of interest require an integration of several techniques making the experiment highly complex however. The most obvious complication is the analysis of the data. To determine the membrane parameters underlying the eAP shape, a computational simulation capable of computing this shape for given model cells is required. To then constrain the parameters well, eAPs must be recorded from multiple well defined electrode positions and the eAP shape must be accurately measured. As the extracellular potentials are also dependent on the surrounding boundaries, this requires knowledge of the shape of the actual cell and its surroundings for optimal results. Computational programs capable of performing the necessary calculation exist [11], leaving the main challenge of gathering the required information for evaluation. For neural networks cultured on glass in a relatively sparse manner, the environment surrounding each neuron largely comprises of extracellular medium of isotropic electrical properties. This simplifies the calculations and requires only the determination of the shape of the cells in the immediate vicinity of the investigated structure. Using the SICM method, the required topography can be determined by the recording electrode (Figure 4.3 (B)). Due to the computer driven piezo stage, positioning of the electrode after topography acquisition can also be achieved with utmost precision in terms of lateral positions and distances from the membrane. Optical identification of the AIS is possible using immunostaining to label neurofascin, which is present in large quantities in the AIS. Figure 4.3 (A) shows a brightfield image of cultured neurons overlayed with the fluorescent image after staining. Figure 4.3 (B) and (C) show the topographic structure of the AIS and its surroundings at different lateral resolution.

As extracellular potentials evoked by a single cell are on the order of only 10 to $100 \mu \mathrm{V}$, an amplifier capable of switching from the voltage clamp needed for SICM imaging to a sensitive potentiometer was used. (see section 6.1.2)

However, even with a low noise voltmeter, determination of the exact shape of such a small signal with high precision is only possible by averaging over a large number of eAPs at each measuring position. For such averaging to be successful a precise time stamp for each AP is necessary. With the signal-to-noise ratio of a single extracellular recording this is not feasible. Instead a larger reference signal is required to properly overlay the eAPs for averaging. This can be done by measuring the AP with a second electrode at a less sensitive region of the neuron such as the soma, where signals are larger.

In the SICM setup this causes difficulties however. As shown in section 6.1.2 the original version of our system performed the raster scanning by laterally moving the sample under an immobile pipette. This prohibited the use of a second patch pipette, as it would lose contact with the measured cell whenever the scanning pipette moves to another position. For this setup a special holder was devised that allowed the integration of commercially available multi electrode arrays (MEAs) into the SICM setup. In this configuration, the entire array along with the neurons cultured upon it would be laterally moved under the SICM pipette and the relative position of neuron and MEA electrodes remain fixed. MEAs as reference electrodes add several complications however. Neurons have to be cultured in special MEA dishes instead of simple glass coverslips or glass bottom petri dishes. This not only increases the 

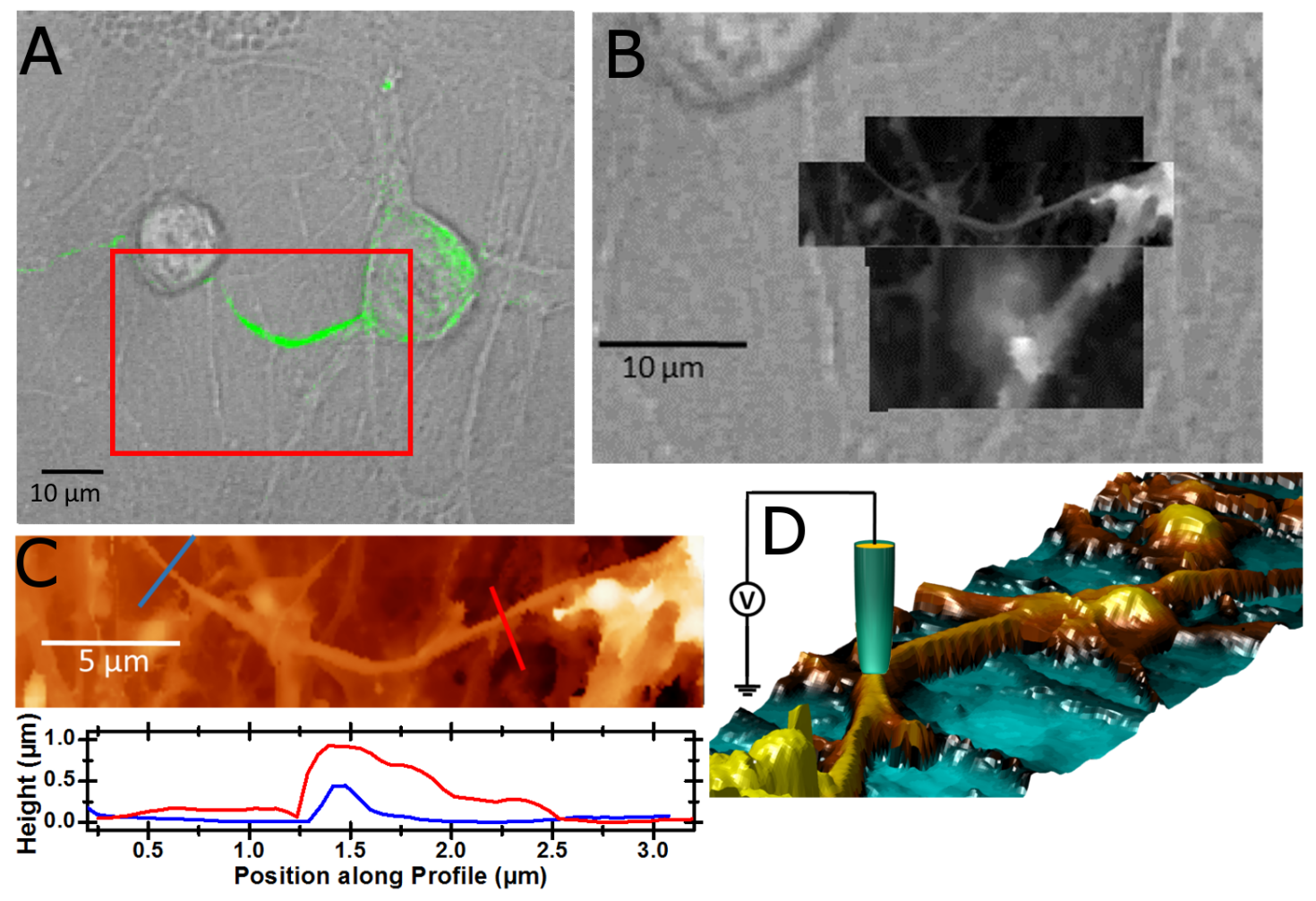

Figure 4.3: AIS investigation. A: By targeting neurofascin with fluorescent dyes, the AIS can be optically identified even with weak optical brightfield contrast. B: Region indicated by the red box in A. By aligning the optical image with the SICM pipette, it is possible to scan the topography of specific regions of the optical image. In this case this was done in two stages: First the surroundings of the AIS were scanned in low resolution to correct for the cell border effects on the extracellular electric potentials in the computer simulations, then the AIS itself was scanned in high resolution for the simulations and the precise positioning of the electrode. C: Close up of the topography in the close vicinity of the AIS along with profiles across the axon. The diameter of the axon at the AIS reaches values below $300 \mathrm{~nm}$ for this cell. This makes patch clamp recordings highly difficult and invasive. As the dimensions of the structure are below the wavelength of optical light, even precise positioning of the recording electrode solemnly guided by optical microscopy is not possible. D: Three dimensional reconstruction of the AIS from the topological data including a representation of the pipette at one of the eAP recording sites. 
costs but also makes optical investigations harder, as low noise MEAs are based on non-transparent titanium-nitride electrodes. As the MEA electrodes record from all cells in close enough proximity, interference from other cells is also possible. Unlike commercial MEA setups, the SICM amplifier can only record from up to two MEA electrodes at the same time and the electrode recorded from can only be changed manually slowing the process of finding a cell providing a good signal. To ensure the high firing rate of the neuron needed for the extensive averaging, it needs to be stimulated during the recording of eAPs. While in principle the MEA electrode can be used for stimulation, it can generally not be used for recording in parallel to stimulation, since the injected charge is so high, and the time constant for discharging is low. For this reason a different stimulation strategy was developed. By fitting the setup with computer controlled high intensity illumination (see section 6.1.2 for details), it is possible to stimulate the cells optically provided they have been transfected with light gated ion channels such as Channelrhodopsin-2.

These difficulties combined with the fact, that software changes to the SICM controller are not possible in this SICM model lead us to pursuit another option based on a newer SICM prototype. In this newer system of our lab, scanning takes place by movement of the recording pipette while keeping the sample at a fixed position. This enables the integration of a second patch pipette for the recording of the reference signal, simplifying the procedure considerably. After acquiring the topographical image of a cell the second pipette can simply be positioned at the cell body by a miniature micro manipulator to record reference APs for time-stamping. Stimulation of the cells is possible either by optical means as in the previous setup or by using the reference electrode for current injection.

\section{Procedure of AIS investigation}

Due to time delays on the SICM manufacturer's part we were not able to automate the system sufficiently to integrate the experimental and computational results. However, the main challenges in regard to acquiring data with the needed precision have been resolved. The following procedure is thus in theory already applicable but unfeasible without custom software due to the requirements of a large number of recordings:

Following the culturing procedure for primary neurons and the life staining of neurofascine as given in section 6.1.4, the glass-bottom petri dish holding the cultured neurons is placed in the SICM holder. A pipette with sufficiently small opening to achieve the required topographical resolution (see section 3.1) is manufactured and inserted into the SICM pipette holder. The micromanipulator is fitted with a patch pipette of about $1 \mathrm{M} \Omega$ for recording of the reference signal. The SICM pipette is then immersed in the bath solution and moved into the field of view of the optical microscope. An appropriate cell in an area with sparse enough cell growth to access the entire AIS is located by bright-field microscopy and the AIS is identified via its fluorescence. At this point, a wide-range topographic SICM image can be acquired. The reference electrode tip is then moved to the soma far from the axon to avoid interference with the SICM pipette. After ensuring that action potentials can be 
triggered in the chosen cell, the AIS and its close vicinity are imaged with SICM in the desired resolution. After the fine topography is mapped, the SICM pipette is moved to different positions which are well defined in lateral direction by the SICM image and in vertical direction by the current reduction due to the increase in access resistance as described in Figure 3.1. At each position the recording mode of the amplifier is switched from voltage clamp to voltage recording and the neuron is stimulated to produce a high firing rate. At this point the SICM pipette is recording many eAPs as illustrated in Figure 4.3 (D). Once the signals are recorded the SICM amplifier is switched back to voltage clamp to move to the next position. This is repeated until enough data is acquired. After the experimental measurement is completed, averaging can be done computationally in the fashion of Figure 4.4. The resulting low noise eAP shapes then need to be compared with simulations. To this end $3 \mathrm{D}$ grids of neurons of the same shape as those recorded from need to be created, so that simulations can be performed under the same boundary conditions. Simulations with different ion channel properties and densities at the AIS are then conducted and those resembling the actual data best are determined.

\section{Future endeavors}

On the experimental side two issues remain to be addressed for ensuring reliable data. One point is the non-optimized software as has already been mentioned in section 4.4. As the system we wanted to use is a one-of-a-kind prototype, the original software shipped with the device had malfunctions making extracellular recordings at a fixed position impossible. Changes to the computer program controlling the SICM could also not be performed by the company in a fast enough manner to facilitate the acquisition of enough data in a sensible timespan. However, in a recent software update to the device the bug prohibiting measurements at a fixed position has been fixed. After some more negotiation we were also provided with the LabVIEW source code, enabling custom modifications to the routines, such as a more advanced adaptive scanning as described in section 6.2.1, a calibration of the optical image compared to the piezo stage as described in section 6.1 .3 and an automated protocol for the extracellular measurement described in section 4.4. The second issue to be addressed concerns the SICM pipette. In the AIS investigation the same pipette has to be used for scanning the sample, determining the distance from the sample and recording the extracellular potential. To achieve high resolution the opening of the pipette should be as small as possible, leading to high resistances. However, for extracellular measurements a low resistance is preferable. This requires a certain compromise which can be dealt with in two different ways. The first is to use pipettes just barely small enough to provide the needed resolution meaning a trade off between spacial resolution and signal strength. The second way is to break off part of the pipette tip after the image acquisition is complete by colliding it with the glass coverslip. It has been shown that for certain geometries fast collisions lead to a clean cut pipette (Y. Korchev, private communication). Our own investigations show, however, that this is rarely achieved if large portions of the tip are removed as is needed to decrease the pipette resistance considerably. This was shown by evaluating the approach curves as described in section 6.1.1. Most of the 


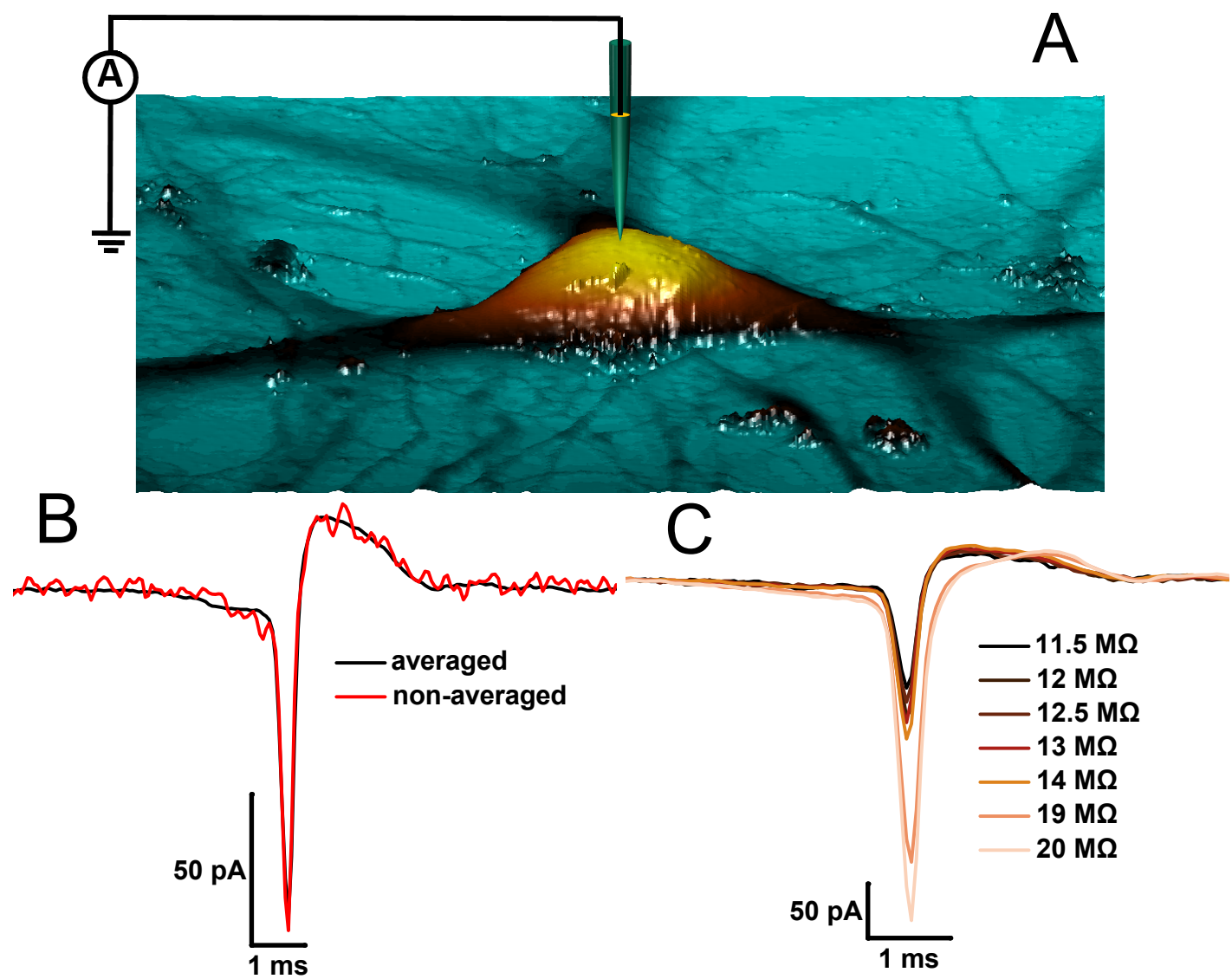

Figure 4.4: Averaging of distance dependent waveform. A: Illustration of measurement arrangement with which the action potentials given in B,C were recorded. Image and AP recording were not performed on the same cell. B: Example of the averaging procedure. In this case, extracellular current measurements at very close distances to the cell body were performed, similar to those proposed as reference measurement in $4.4(\mathrm{n}=33)$. Due to the large signal to noise ratio in these measurements the time stamp needed for averaging can be directly derived from the pulses themselves. C: Averaged traces of the AP current at different distances from the cell indicated here by the different access resistances. The detected current increases with closer proximity to the membrane. In addition a change in the shape is clearly visible for the two closest distances. The same electrode was used to record all traces. 
time, the pipette tip was not smooth enough to achieve a current reduction of more than $40 \%$ with pipettes broken to more than twice the original conductance. Some improvement is possible by driving the pipette tip quickly onto the glass multiple times as this can chisel away some of the uneven parts. This loses effectiveness as the pipette wall gets thicker however and current reduction of more than $40 \%$ are still rarely possible. The uneven opening of such pipettes adds uncertainty to the position and shape of the electrode during the eAP recordings. However, as shown in section 6.1.1, the distance between the pipette opening and the sample is influenced only weakly by lower quality tip shapes. 


\section{Characterization of the channelrhodopsins Chronos and ChR2}

\section{Motivation}

Analysis of the extracellular action potential waveform requires absolute precision and accurate knowledge of all involved components. During the course of this thesis it was attempted to characterize the relevant processes with as much detail as possible. Section 6.1.1 deals with problems arising from the pipette geometry and section 6.1.2 with the high requirements on the amplifiers. As explained in section 4.4, our original setup prohibits the use of a second patch pipette, making optical stimulation of our samples the most feasible technique. Consequently, we wanted to also achieve optimal stimulation precision using optical means.

Making use of the versatility of light for exciting neurons is an intriguing idea due to the possibility to illuminate small regions [70, 71, fast switching, quick spacial relocation of the stimulation site [71] and the low requirements for mechanical stability [72]. Successful attempts were already reported in 1971 [73]. Since then more sophisticated methods for excitation by light were developed such as glutamate uncaging [74] or the modification of ion channels to provide an optical switch [75]. However, the only method offering sustained precisely timed spike trains is the use of channelrhodopsins (ChRs) [76], a group of naturally occurring photosensitive ion channels i.e. membrane proteins that increase their conductance after photon absorption.

Especially the discovery of Channelrhodopsin-2 (ChR2) in the green algae Chlamydomonas reinhardtii [77, 78, has ushered in a new area of optical stimulation of electrically excitable cells. Due to its relatively fast open kinetics this cation specific light-gated ion channel is able to depolarize cells with temporal precision and a low invasiveness not achievable before. Additionally it has paved the way to a new method of neural stimulation termed 'optogenetics' due to the fact, that only cells transfected with the gene of the light-gated channel will respond to the light stimulation. This enables the experimenter to employ genetic targeting of certain cell types, providing the means to easily probe the influence of subpopulations of neurons [79]. It also enables the creation of stable cell lines and even transgenic animals expressing the light channel in certain cell types [80].

ChR2 offers high transfection levels, response times on the millisecond scale and currents able to trigger action potentials. While this is sufficient in many cases, efforts have been made to improve the performance in various aspects to meet the need of specific experiments [81, 82]. For triggering precisely timed action potentials for example, it is necessary to achieve a certain total depolarization quickly, which 
means the conductance has to change quickly and to a large degree. For other applications a slow deactivation of the current is preferred so that excitation with weak short light pulses is possible. Variants with extremely long deactivation times are used as step function switches [83]. For any application that requires high frequency responses both the onset- and deactivation of the conductance change should be fast.

To provide the most precise stimulation we decided to use 'Chronos', the fastest light-gated channel known today. Due to the superb kinetics of said channel it should provide the fastest response and the most precise excitation in neurons. The initial investigation started as a bachelor student project by Hanna Gottschalk who determined the time constants of Chronos' current response in human embryonic kidney cells. In her thesis Hanna Gottschalk showed that at low intensities Chronos does indeed possess the fast kinetics described earlier [84]. Ricardo Martins Merino (MPI DS, Göttingen) performed additional investigations on Chronos concerning its temperature dependence and the viability of usage in neurons. He found, that at physiological temperatures the currents mediated by Chronos are able to follow light stimulation for frequencies of more than $100 \mathrm{~Hz}$ and the rise time is fast enough to emulate even fast synapse currents. His work on neurons shows however, that transfection with Chronos is less reproducible than with the widely used ChR2 (personal communication). This begs the question if an engineered variant combining the kinetics of Chronos with the reliability of ChR2 can be created. To this end a characterization beyond the mere performance investigation is required and function/structure relations need to be resolved. This chapter describes how I tackled this problem by creating a detailed, physically plausible state model applicable to both ChR2 and Chronos and comparing the differences using computer simulation inspired by a method previously used by Andreas Neef on voltage-gates channels.

\section{History of ChR characterization}

The structure of the channels comprises seven trans-membrane helices providing a binding pocket for the native retinal of the cell. Retinal is covalently bound to the construct in its all-trans form, opposed to its natural dark state in mammals which is the 11-cis state. Absorption of light by the retinal leads to a deprotonization of the retinal Schiff base and a conformational change from the all-trans into the 13-cis conformation. This in turn leads to a number of electronic and conformational changes in the ChR molecule involving a state possessing highly increased conductivity for cations. In its wild type form this 'open' state is not stable, and the molecule will decay back into its pre-illumination state with the retinal re-protonating and able to re-run the light cycle upon further illumination.

Optical stimulation and optical readout of absorption changes can be performed with relatively low effort and at several wavelengths. Such spectroscopic studies have produced a wealth of information about the gating of channelrhodopsin (ChR) [85, 86, 87, 88], down to the sub-microsecond range. Early spectroscopic studies on ChR2 managed to distinguish a total of five different states along with the associated lifetimes from the difference in their absorption spectra using visible light. Two of these states were considered to be conducting and one transition could be at- 
tributed to the deprotonation of the retinal Schiff base [85]. A later study, combining measurements in the ultraviolet/visible spectrum with IR-Spectroscopy, managed to distinguish six states and associated each with molecular changes of the retinal and the channel itself such as the changes in bonding of the amino acid E90, a process considered to be crucial for gating in ChR2 [86].

The results are shown in Figure 5.1 for ChR2.

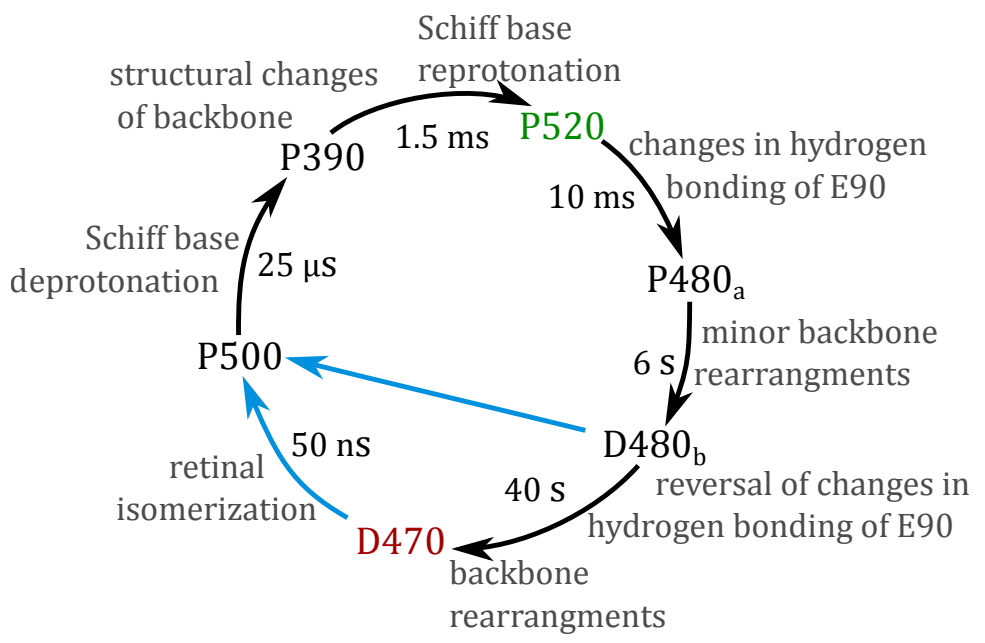

Figure 5.1: Light cycle of ChR2 as derived from optical spectroscopy. Light independent transitions are indicated by black arrows while light induced transitions are shown in blue. Transition time constants and the molecular changes underlying the absorption spectra are given. Except for a light induced transition from $D 480_{b}$ a purely linear cycle is assumed. The ground state is marked in red and the conducting state in green. Adapted from [86].

Even before the first spectroscopic analysis of ChR2, electrophysiological approaches have been applied to gain insight into the light cycle of the protein. These investigations used well known principles from the study of voltage-gates channels and transfered these approaches to light-gated proteins. Light-stimulation protocols probing for hidden (that is to say non-conductive) states by making use of the changes in the waveform were employed. Limited by the time resolution of electrophysiological measurement, these studies have nevertheless improved the knowledge about some key aspects of the ChR light cycle and have even extended the light cycles derived from spectroscopy by showing that more than one conducting state exists in ChR2. Electrophysiological characterizations of channelrhodopsin gating also have the advantage that they look at the one parameter most relevant to the application of the tool: the conductance. They also put enough constrains on the possible state maps underlying the observed current waveforms to show that a second, parallel level needs to be added to the simple cyclic model as was first shown in [89] for ChR1. Spectroscopy seems to be insensitive to these difference of sequential and parallel reactions, as well as to the different conductances between the two levels from which the electrophysiological differences seem to stem 90. It seems that a combination of spectroscopic and electrophysiological techniques would be optimal 
to construct a comprehensive model of channelrhodopsin gating that accounts for transitions in absorption and conductance during microseconds to seconds of illumination. However, the electrophysiology and spectroscopy are based on different preparation techniques, and to my knowledge combined experiments have so far not been reported. Furthermore, the few studies that described channelrhodopsin currents with the proposed double cycle model have not aimed at a biophysically motivated description but instead aimed at minimal model complexity [91, 92, 47]. A model commonly used for simulations in the literature is given in Figure 5.2. It possesses only two open and two ground states [91, 92, 47]. One open/ground state pair represents the light adapted and the other the dark adapted cycle. Light dependent transitions exist between each ground state and the corresponding open state as well as between the two open states.

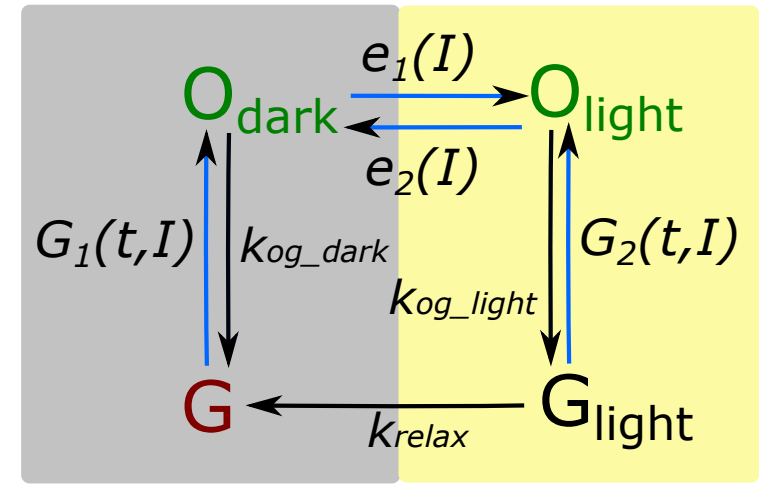

Figure 5.2: Branched model as seen in the literature. The branching model with the lowest number of states consists of a dark adapted cycle (gray shaded) and a light adapted cycle (yellow shaded). Each cycle consists of only one open and one closed state. In order to represent the real waveforms with such few states, the complex transitions given in equation 5.0.1 and equation 5.0.2 are used. Adapted from [91].

The latter leads to a transition between the light and dark adapted cycles. Without illumination however the only possible transition between the two light cycles is from the light adapted ground state to the dark adapted ground state. The speed of this transition corresponds to the speed of the recovery from light adaptation an effect illustrated in Figure 5.6. The open states decay thermally into the corresponding ground states.

To reproduce the currents of the actual channel with a model consisting only of four states and 7 transitions, additional complexity was integrated into those transitions at two stages: In the first stage, an intermediate state between ground and open state was subsumed into the open state using a time dependent transition rate. Within the limits of a constant excitation rate, this approximation is exact and the influence of the intermediate state can still be extracted. However, responses to continuously time-varying stimuli cannot be described. In the second stage, a number of transitions with constant rates or rates with linear light dependence are subsumed into the two transitions between the open states so that a very complex, 
heuristically chosen light dependence emerges. This leads to a good representation of the current, but disconnects the computational model from the molecular states, making it practically impossible to relate features of the observed current waveform to individual molecular transitions. The exact equations used are as follows:

$$
G_{i}(t, I)= \begin{cases}\alpha I\left[1-\exp \left(-t / \tau_{C h R}\right)\right], & \text { for } t<t_{\text {light }} \\ \alpha I\left[\exp \left(\left(t-t_{\text {light }}\right) / \tau_{C h R}\right)-\exp \left(-t / \tau_{C h R}\right)\right], & \text { for } t>t_{\text {light }}\end{cases}
$$

for the excitation, and

$$
e_{i}(I)=k_{\text {dark }}^{i}+c_{i} \log \left(1+I / I_{0}\right)
$$

for the adaptation. With $\alpha$ being the effective absorbance, $\tau_{C h R}$ the time needed for the conformation change into the open state, $t_{\text {light }}$ the duration of the light pulse, $k_{d a r k}^{i}$ the transition rate between open states in the dark and $c_{i} \& I_{0}$ free constants. In contrast to the before-mentioned model, this study sets out to provide a biophysically motivated Markov model of light-activated ion channels, capable of capturing the kinetics of molecular transitions and applicable to different channelrhodopsin variants. As an exact state/transition map is not a priori available, the strategy to obtain a map is systematically increasing the complexity of the model to account for different effects and then globally optimize the model computationally taking all effects into account.

A Markov model is most useful for an understanding of structure/function relations, if it successfully captures the properties of different members of the Channelrhodopsin family. We therefore studied ChR2, the most widely used optogenetic tool [93], in addition to the very recently introduced variant Chronos [84], which possesses gating kinetics clearly faster than that of any previously developed variant. As Chronos is found in $\mathrm{S}$. helveticum while ChR2 is native to Chlamydomonas reinhardtii, significant structural differences can be expected. Yet the same Markovmodel structure can describe both channels, indicating that the transition scheme of our model captures general processes in ChRs, enabling a direct comparison. The main cause for Chronos' faster kinetics in this model is a faster decay of the open state compared to ChR2. This makes it possible for Chronos to reach its peak current, at which the number of molecules entering the conducting state equals those leaving it, more quickly. In consequence, a quicker activation and deactivation is possible at the cost of having fewer molecules in the conducting state i.e. lowering the opening probability at a given intensity. In our simulations this reduction is dependent on the intensity of the stimulation and the opening probability of Chronos is between $20 \%$ and $50 \%$ of ChR2's. 


\subsection{Results}

\subsubsection{Time constants of the current waveform response}

Several key properties of ChR kinetics can be extracted from whole-cell recordings of current waveforms evoked by a single long square light pulse: the rise time, the decay kinetics towards the steady state level and the decay time in darkness. Figure 5.3 shows such a current response for both Chronos and ChR2 to 1 second light pulses of $1 \mathrm{~mW} / \mathrm{mm}^{2}$ and $7 \mathrm{~mW} / \mathrm{mm}^{2}$. The general shape of the response is similar for both proteins, with an initial peak followed by an equilibrium current settling after several 100 milliseconds. Chronos' current exhibited a faster rise after illumination onset and fall after the stimulus ended as well as a narrower transient. Figure 5.3 (C) gives a direct comparison between the activation time-constants of ChR2 and Chronos, defined here as the time constant of a single exponential fit to the initial rise of the current. The current rise for Chronos is considerably faster than for ChR2 at all studied intensities. This is especially interesting for low intensities at which the activation is slow for ChR2 and several of its derivatives. For applications employing unfocused light sources such as the LED we designed for easy integration into standard microscopes (see section 6.1.2), using Chronos can thus have considerable advantages, as the activation time-constant of ChR2 can reach several milliseconds for light intensities below $1 \mathrm{~mW} / \mathrm{mm}^{2}$. The speed of the activation and decay kinetics can be crucial to an experiment, as they are among the factors limiting the firing frequencies achieved in neural stimulation [81. The other major factor is the current amplitude determined by the expression level, the single channel conductance and the open probability. The latter is connected to the kinetics through the life times of the molecular states as will be shown in section 5.1.5.

\subsubsection{Early intermediates of the light cycle}

While the time constants of the current waveform such as the activation in Figure 5.3 (C) are valuable for experimental considerations, they do not directly reflect single molecular transitions in the light cycle. This is due to the complex involvement of many intermediates of the light cycle during constant illumination. However, employing very short pulses leads to a response dominated by the early intermediates of the photo cycle and any two photon effects caused by later photo-sensitive intermediates are strongly reduced. A simple three state model with a ground state, an excited state and a conducting/open state as shown in the inset of Figure 5.4 (A) is sufficient to reproduce the responses to light pulses of less than $100 \mu$ s duration. Assuming a pure thermal relaxation leading to an exponential decay of the excited state $E$ yields the following derivative of the occupancy of said state:

$$
\frac{E(t)}{d t}=-\frac{E(t)}{\tau_{e}}
$$

Where $\tau_{e}$ represents the life time of the excited state. The open state $O$ will be populated from $E$, so its change in population is given by 

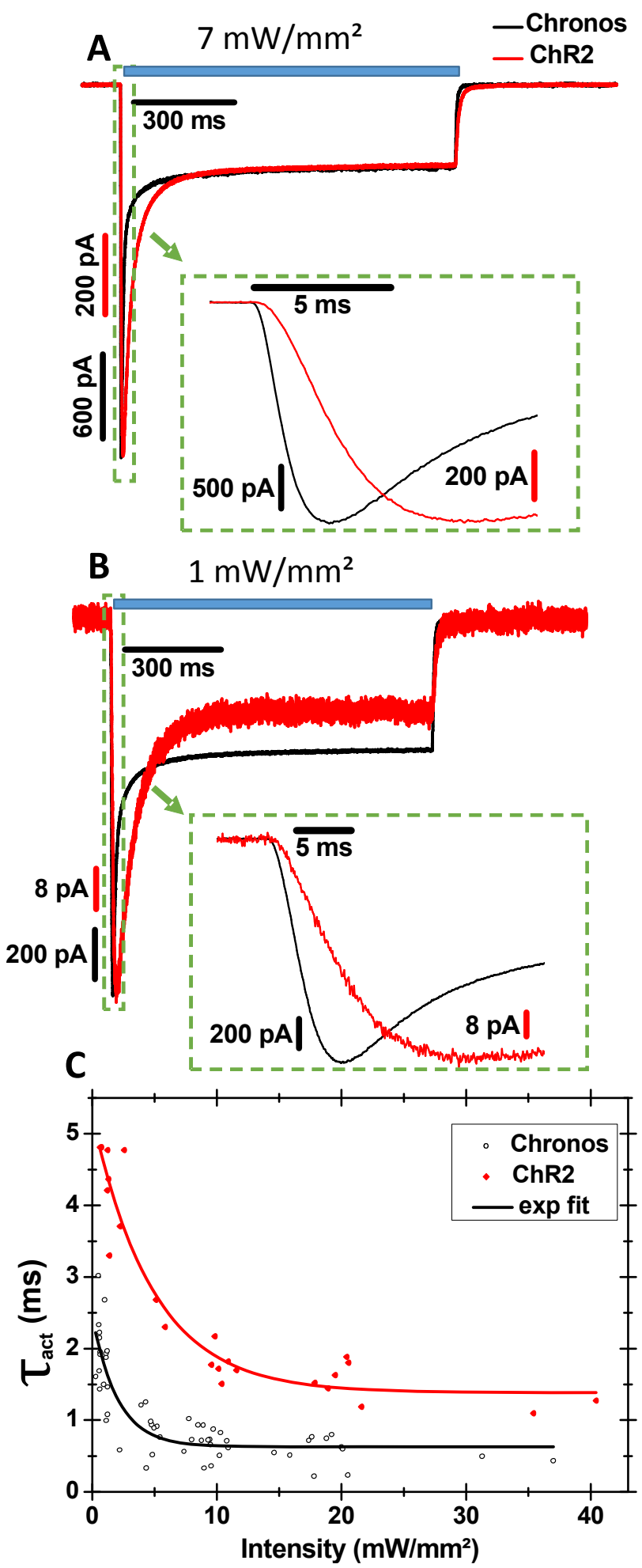

Figure 5.3: Chronos displays faster light response than ChR2. A/B: Current response of Chronos (black) and ChR2 (red) to a 1 second square light pulse (indicated by blue bar). All three time constants associated with the typical ChR response (e.g. activation, transition to the steady state and deactivation in the dark) are smaller for Chronos, enabling a quicker depolarization of the cell and high-frequency stimulation. Insets show the activation phase in more detail. As can be seen from the scale bar, the time needed to reach the peak current is strongly reduced for higher light intensities. (A). Panel C shows this in a quantitative way. Here the time constant of the rising current is plotted against the light intensity for cells expressing Chronos $(n=9)$ and ChR2 $(n=4)$. Red diamonds represent $\mathrm{ChR} 2$ and black circles Chronos. Lines are exponential functions fitted to the data-points. 


$$
\frac{O(t)}{d t}=\frac{E(t)}{\tau_{e}}-\frac{O(t)}{\tau_{o}}
$$

with $\tau_{o}$ being the life time of the open state.

Since flashes below $100 \mu$ s are short compared to the response, the time course of the excitation is not relevant and $E$ will not have time to decay notably during the duration of the pulse. It can thus be assumed that at $t=0$ the excited state $E$ is populated to a certain degree $E_{0}$ depending on the number of photons absorbed during the light pulse. At $\mathrm{t}=0$ the states are thus populated in the following way $O(t=0)=0$ and $E(t=0)=E_{0}$.

Which leads to a simple exponential decay for $\mathrm{E}$

$$
E(t)=E_{0} \exp \left(-\frac{t}{\tau_{e}}\right) .
$$

This means the change of $\mathrm{O}$ becomes

$$
\frac{O(t)}{d t}=E_{0} \exp \left(-\frac{t}{\tau_{e}}\right)-\frac{O(t)}{\tau_{o}} .
$$

Which is being solved by a bi-exponential decay function

$$
O(t)=\frac{E_{0}}{\tau_{e} / \tau_{o}-1}\left(\exp \left(\frac{t}{\tau_{e}}\right)-\exp \left(\frac{t}{\tau_{o}}\right)\right) .
$$

The initial occupancy $E_{0}$ depends on the total light energy $I \cdot \Delta t$ of the pulse in the following way:

$$
E_{0}(I \cdot \Delta t)=1-e^{-I \Delta t \alpha}
$$

With $\alpha$ being the effective absorption constant of the molecule and $I$ the light intensity. Figure 5.4 (A) shows the response of a cell transfected with Chronos to a flash of light and the fit according to equation 5.1.1. Importantly, the rise of the current continues after the stimulus ends. Therefore it is possible to estimate a value for the time constant $\tau_{e}$ which states how fast the molecules change from the excited into the conducting state.

The fits of pulse driven currents indicate, that the decay of the open state $\tau_{o}$ is more than a factor of two faster for Chronos than it is for ChR2. These values are nearly identical to the deactivation times obtained from fitting the decay of the current after a long pulse with an exponential function. This is to be expected if no longlived photo-cycle intermediates exist before the open state. However, if more than one conducting intermediate exist, the deactivation time would be a combination of the decay times of those conducting states. The second time constant $\tau_{e}$ obtained from a single short pulse is also about two fold faster for Chronos than for ChR2 implying that Chronos can enter the conducting state faster than ChR2.

The total number of photons in the pulsed stimulus can be varied by means of the light intensity $I$ or the pulse duration $\Delta t$. This makes it possible to estimate the absorption-constant $\alpha$ by applying equation 5.1.2. Longer pulses cause more deviation from the approximation that excitation is instantaneous however, which leads 

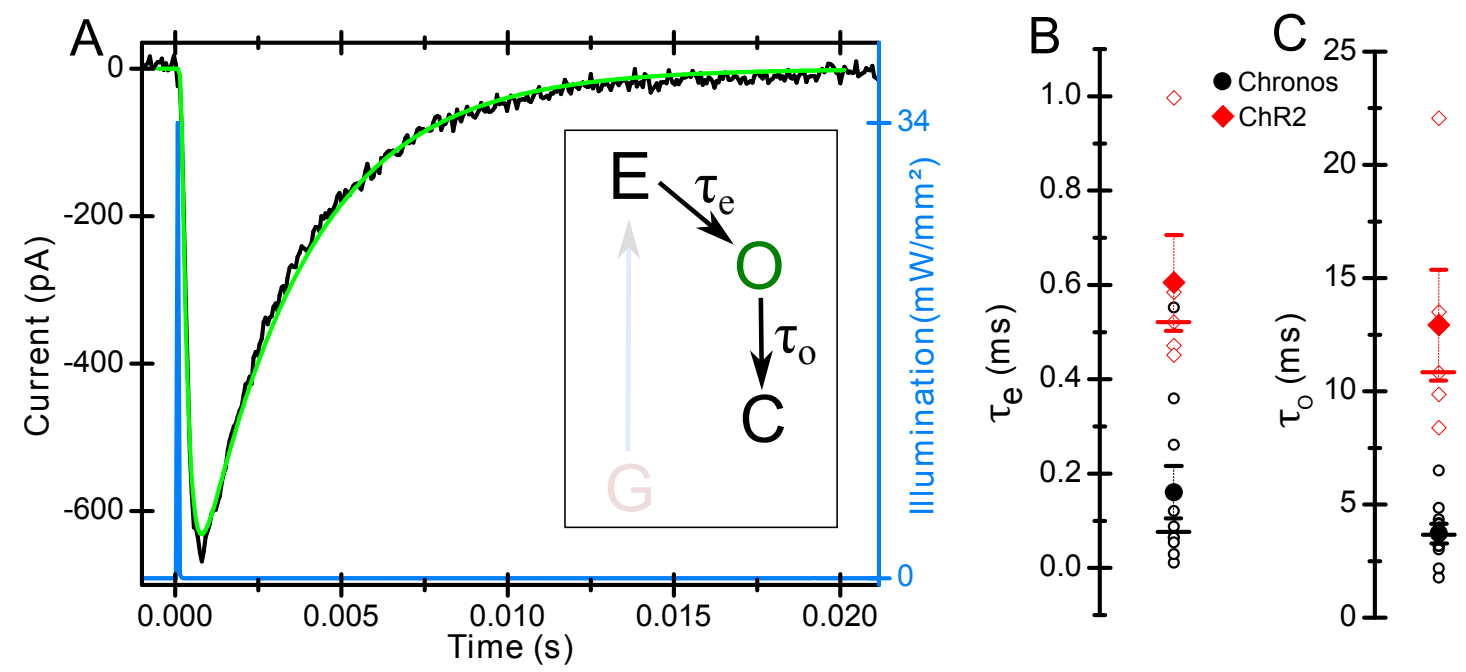

Figure 5.4: Impulse Response. A: Current response of a cell transfected with Chronos (black) to a $60 \mu$ s light pulse (blue) fitted with Equation 5.1.1 (green). The duration of the pulse is negligible as the current is increasing noticeably only after illumination ended. Inset: Markov Model used to evaluate the response to short pulses. As later intermediates of the light cycle do not influence the response to a short pulse, even a simple model comprising of only an excited state $E$, an open state $O$ and a closed state $C$ describes the data well. B/C: Parameters of the model for both Chronos (black circles) and ChR2 (red diamonds). Small symbols represent the data points, while the larger, filled ones represent the mean. Long horizontal lines show the median while short lines show the standard error of the mean. As can be seen, the decay time of the excited state (B) and that of the open state $(\mathbf{C})$ of Chronos $(n=10)$ is faster than that of ChR2 $(n=5)$. 

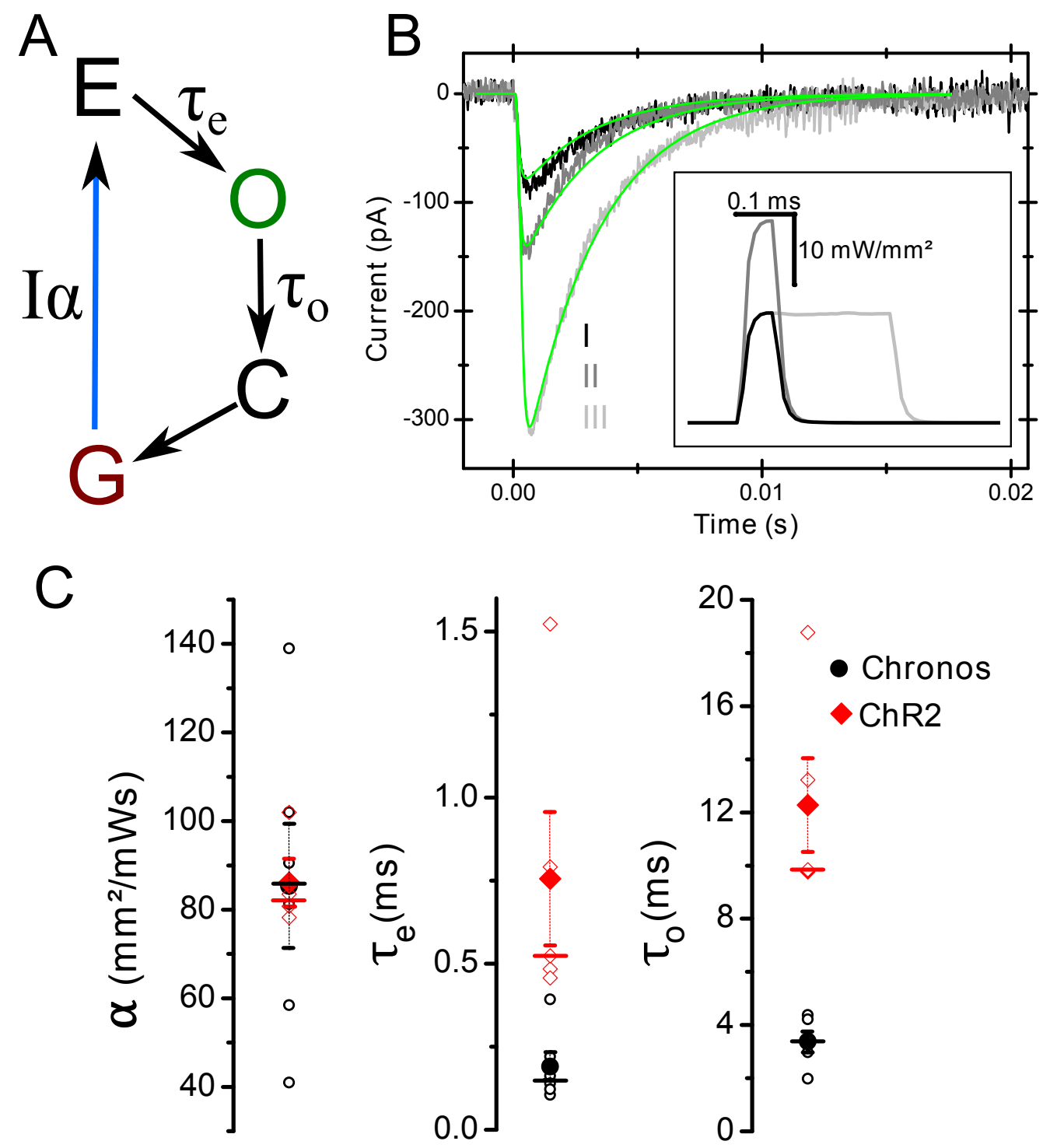

Figure 5.5: Simple closed loop model. A: Model used to simulate the current response waveform. It encompasses the three state model of Figure 5.4 but closes the loop through the ground state $\mathrm{G}$ from which light induced excitation takes place. B: Current response of a cell transfected with Chronos (gray) to three light pulses with different total energy. The simulation with global parameters is overlaid in green. The inset shows the light pulses recorded during the experiments which were also used for the simulations in the same color as the corresponding current trace. C: Parameters extracted from the optimized simulation for Chronos (black circles) and ChR2 (red diamonds). Small symbols represent the data points, while the larger, filled ones represent the mean. Long horizontal lines show the median while short lines show the standard error of the mean. 
to a worse approximation by equation 5.1.1. For this reason, the more practical approach is to determine the parameters by simulating a simple closed-loop lightcycle as shown in Figure 5.5 (A). This way the effect of non-instantaneous excitation caused by longer light pulses is accounted for, since at each time point during the pulse the population $E$ increases according to the pulse intensity. The absorbances $\alpha$ for ChR2 and Chronos acquired in this fashion do not significantly differ (Figure 5.5 (C)). However, the transition to the open state (characterized by $\tau_{e}$ ) as well as the closing of the channel $\left(\tau_{o}\right)$ are faster for Chronos, consistent with the single pulse result from the analytical analysis above. Both parameters influence the fast current response of Chronos observed in Figure 5.3 (C). A fast transition to the conducting state as characterized by $\tau_{e}$ leads to a quicker response of the channel and it is reasonable to assume that this transition can limit the activation speed at high intensities. However, the sub millisecond nature of $\tau_{e}$ for both ChR2 and Chronos leads to an excited state $E$ which is quickly depopulated for both channels at low intensities. The faster decay of the open state also contributes to the activation speed, as it makes it possible for Chronos to reach its peak current, at which the number of molecules entering the conducting state equals that leaving it, more quickly. This leads to a quicker activation at the cost of fewer molecules in the open state. As the simple model does not reproduce the response to long light pulses well, it is not possible to deduce the exact effect of each time constant from pulsed experiments and strongly reduced models alone even under the assumption that one of the molecular transition probed with short pulses dominates this effect. This is why in the following the model will be extended on the basis of phenomena seen during longer light pulses.

\subsubsection{Challenges for a complete model/effects of long pulses}

Another characteristic property of $\mathrm{ChR} 2$ is the light adaptation first reported in [78. It describes the reduction of the characteristic peak of the current response for several seconds after a prior illumination and is thus not directly observable during single-pulse stimulation. Interestingly this reduction is specific to the peak and does not influence the steady state reached several 100 milliseconds after pulse onset as can be observed in Figure 5.6 (A) for Chronos. ChR2 exhibits this effect in a far stronger manner than Chronos. The peak for Chronos is never reduced by more than $40 \%$ of the original current while for ChR2 more than $60 \%$ reduction is visible. With a time constant of $\sim 3 \mathrm{~s}$, Chronos also recovers about twice as fast to the fully dark adapted level compared to ChR2 which has a time constant of $\sim 7 \mathrm{~s}$ in our conditions. In our experiments using a membrane potential of $-60 \mathrm{mV}$, light adaptation was mostly intensity independent for both ChR2 and Chronos (data not shown). Williams et al. showed however, that at higher (less negative) voltages a mild speed up of the recovery for higher intensities exists [47].

From an experimental point of view this is an important feature. If the channels are used to drive spikes as in the procedure described in section 4.4 , then the decreased sensitivity during light adaptation has to be taken into account during the design of light-stimulation waveforms. 

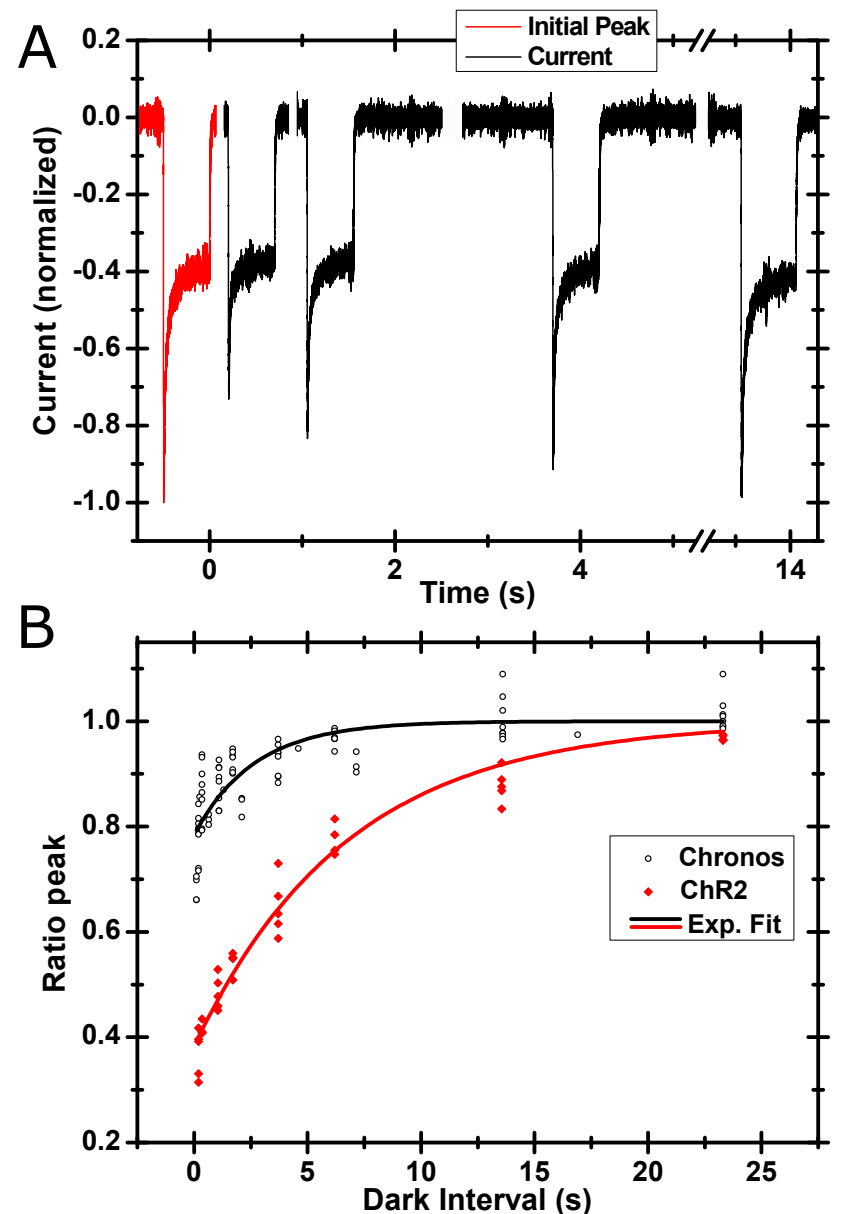

Figure 5.6: Light adaptation. A: Current response showing light adaptation for Chronos. Two identical square light pulses separated by varying dark times stimulate the cell. The response to the second pulse (black) shows a reduced peak, while the steady state remains unchanged. The red curve indicates the first of the two stimuli. A 30 s dark phase preceded each sweep so that the first pulse reaches the same peak current for every trial. B: Ratio of peak current for two subsequent square light pulses plotted against the dark time in between the pulses. This effect is especially pronounced in ChR2 (red diamonds) where it has been attributed to faster transitions between the light adapted and dark adapted states during illumination than in the absence of light. The red and black curves show exponential fits to estimate the time constant for the recovery. It is about $3 \mathrm{~s}$ for Chronos (black circles) and $7 \mathrm{~s}$ for ChR2. 
A simple circular model as presented in Figure 5.5 (A) is unable to reproduce the current response for all light stimuli. This becomes especially apparent when considering the light adaptation as shown in Figure 5.6. Here the slow recovery with timescales of several seconds would require a molecular transition of similar timescale. In a one cycle model however this cannot be achieved without slowing down the time required to reach equilibrium as well. In addition, the single-cycle model does not faithfully reproduce the shape of the current response to long light stimulation, especially when the pulses encompass a wide range of intensities. For this reason, models of the molecular states of ChR2 were modified from purely circular sequential models to branching models allowing equilibrium to be reached faster during illumination than in the dark, as was first introduced by Hegemann et al. for ChR1 89. In these branching models two conducting states belonging to different light cycles exist [89, 78]. (see Figure 5.2) By implementing either a lower conductance for the light adapted open state or a slower pathway to that state in the light adapted pathway, the initial peak is reduced after prior illumination. A fast transition from the light adapted to the dark adapted cycle which can only take place during illumination is also present to achieve the same steady state after light adaptation. Another slow transition mediates the relaxation in the absence of illumination clearly observable in Figure 5.6.

This study evaluated a number of circular and branching models, and as previous studies indicated, only the branching models represent experimental data well, even if the stimulation used does not probe specifically for light adaptation. The basic structure for all the branching models comprised of two light cycles identical in structure to that given in Figure 5.5 (A). This basic scheme is used due to the fact that the main structure of the system, i.e. the seven trans-membrane helices creating the pore and the retinal inside its binding pocket, does not change during light adaptation. The molecular changes responsible for gating after light absorption are thus likely to be similar, even if the actual rates can differ as even small changes in the electronic environment of the retinal and key amino acids involved in gating are known to have strong influence on the kinetics [83, 94, 81, 95]. This basic structure still leaves room for a number of different Markov-models, for example by having a different connectivity between the two loops. In order to constrain the possibilities of connections, several considerations and tests have been performed. First of all, simulations showed that models having only one pathway from a specific state to the light adapted cycle gave better results than those assuming that molecules could become light adapted independent of their current electronic state. Models in which this transition takes place from the open state managed to reproduce recorded data the best. The fact, that short light flashes cannot induce light adaptation as stated in [90] indicates that this transition is light induced. A back transition from the light adapted open state has some strong logical constrains if the model is to be physically realistic. This transition cannot be of a purely thermal nature while the dark adapted open state has greater conductance than the light adapted one. If it were, molecules would only transition from the light-adapted to the dark-adapted open state after the light is switched off. This would result in a peak in current, an effect not observed. A light induced back-transition on the other hand would only 
be energetically possible with additional intermediate states. I have thus chosen a light independent back transition from the light adapted cycle between the two non-conduction states $C_{\text {dark }}$ and $C_{\text {light }}$.

\subsubsection{Evaluation via simulation using a complete model}

Figure 5.7 presents the model giving the best results under the tested conditions. In the totally dark adapted state all molecules are in the ground state $G$. Only by traversing the conducting state $O_{\text {dark }}$ can a molecule change into the light adapted cycle via the transition characterized by the constant $k_{\text {adaption }}$. During constant illumination the two light-cycles reach equilibrium. After illumination ends, molecules gather in the dark adapted ground state and in the state $G_{\text {light }}$, from which they decay only slowly back into the ground state through $k_{\text {relaxtoground }}$. In this model light adaptation exists if the cell is illuminated before all molecules have transitioned from $G_{\text {light }}$ into the ground state $G$. In this case the light adapted open state $O_{\text {light }}$ is not only reached through the other conducting state, but also by excitation from $G_{\text {light }}$. This changes the current response during the peak phase but not the equilibrium.

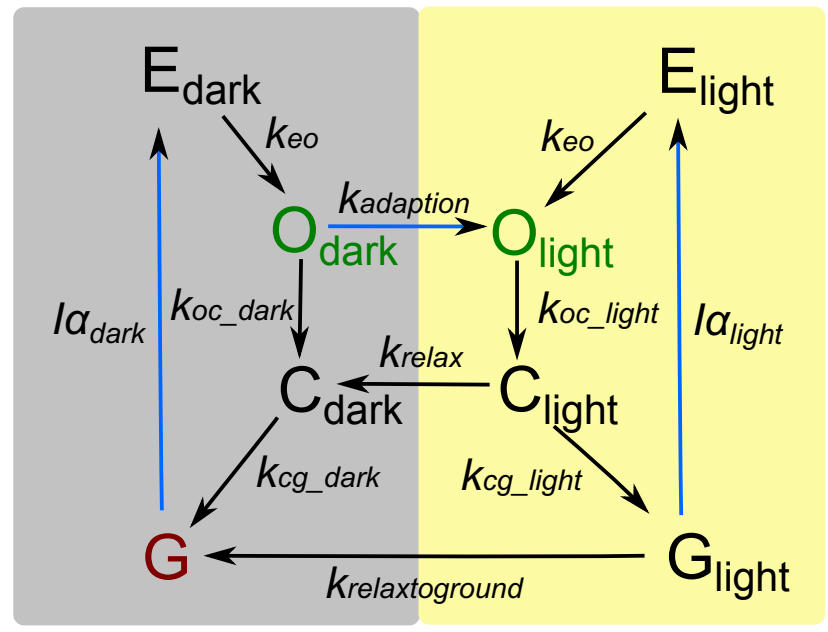

Figure 5.7: Markov model of the light cycle of ChR2/Chronos. The branching model consists of two structurally identical sub-cycles reaching equilibrium during illumination at constant intensities due to the transitions between the two open states $O_{\text {dark }}$ and $O_{\text {light }}$ and the two closed states $C_{\text {dark }}$ and $C_{\text {light }}$. We term the yellow highlighted sub-cycle 'light adapted cycle', since its 'ground state' $G_{\text {light }}$ is only populated for a certain time after illumination. Without illumination the transition $k_{\text {relaxtoground }}$ leads to a slow relaxation to the totally dark adapted state. The gray shaded dark-adapted cycle is equivalent to the non-branching model of Figure 5.5. All black arrows indicate transitions consisting of exponential decay, while the blue arrows indicate transitions through linear absorption.

It is important to note, that the transition represented by $k_{\text {adaption }}$ determines how much the single cycle approximation deviates from the branching model of Figure 5.7. As the transition is light dependent in our model, short pulses cannot 
induce light adaptation from $O_{\text {dark }}$ and the values gathered from the pulsed experiments should therefore be good approximations.

As the light adapted ground state $G_{\text {light }}$ is never fully populated in this model, the strategy of probing light adapted transitions with short light flashes is not as straightforward as for the dark adapted cycle. However, by comparing simulated data for more complex stimuli to recorded data, the transition rates between molecular states can be extracted even from subtle features in the current waveform. By using several different intensities and stimulation protocols including clear light adaptation, the model parameters are tightly confined. Using the values gathered from the pulsed experiments as starting points for the dark adapted cycle, parameters are globally optimized by simulating curves for all the light stimuli and calculating the total derivation between the simulated and recorded curves. A genetic algorithm reduces the chance of finding only local minima in parameter space during the minimization of the squared error between simulation and recorded data. Except for parameters predefined by other experiments as those with short light pulses, a large range of about three orders of magnitude was allowed for each parameter to allow unbiased optimization.

Figure 5.8 shows a comparison between the recorded data and the simulated curves with optimized parameters for both Chronos and ChR2 while Table 5.1 list the parameter values of the model.

\subsubsection{Influence of single transitions on waveform}

Most effects seen in the current waveform depend on the properties of many molecular transitions in a complicated manner. Thus identifying which transitions are mainly responsible for differences in ensemble properties such as activation speed is difficult. Exchanging certain parameters obtained from ChR2 with those received from Chronos, while keeping all other transitions the same is one method to address this problem. Simulations with these 'hybrid' parameter sets give insight into the extent to which certain transitions influence a specific population property. This way the question which parameter is mostly responsible for activation speed can be addressed. The $k_{e o}$ transitions of the two cycle model correspond to the time constant $\tau_{e}$ in the one cycle model, while $k_{o c}$ corresponds to $\tau_{o}$. Exchanging these parameters in the ChR2 parameter set with those from Chronos reveals if the increased transition speeds observed during pulsed experiments play a role for longer light stimulation. The response of the hybrids to a $1 \mathrm{~s}$ square light stimulus with an intensity of $2 \mathrm{~mW} / \mathrm{mm}^{2}$ as well as the effect of exchanging either $k_{e o}$ or $k_{o c}$ on the activation time as determined by an exponential fit to the simulated curves is provided in Figure 5.9 .

The response of the hybrid with a faster decay of the excited state $k_{e o}$ only differs slightly from that of the pure ChR2 parameter set. This is due to the fact, that even though this value is nearly twice as large for Chronos, it is still by far the fastest transition in the light cycle of ChR2. This leads to an almost completely unpopulated excited state $E$ during most of the simulation. Only shortly after the onset of high intensity illumination is $E$ populated enough to see an effect in the 

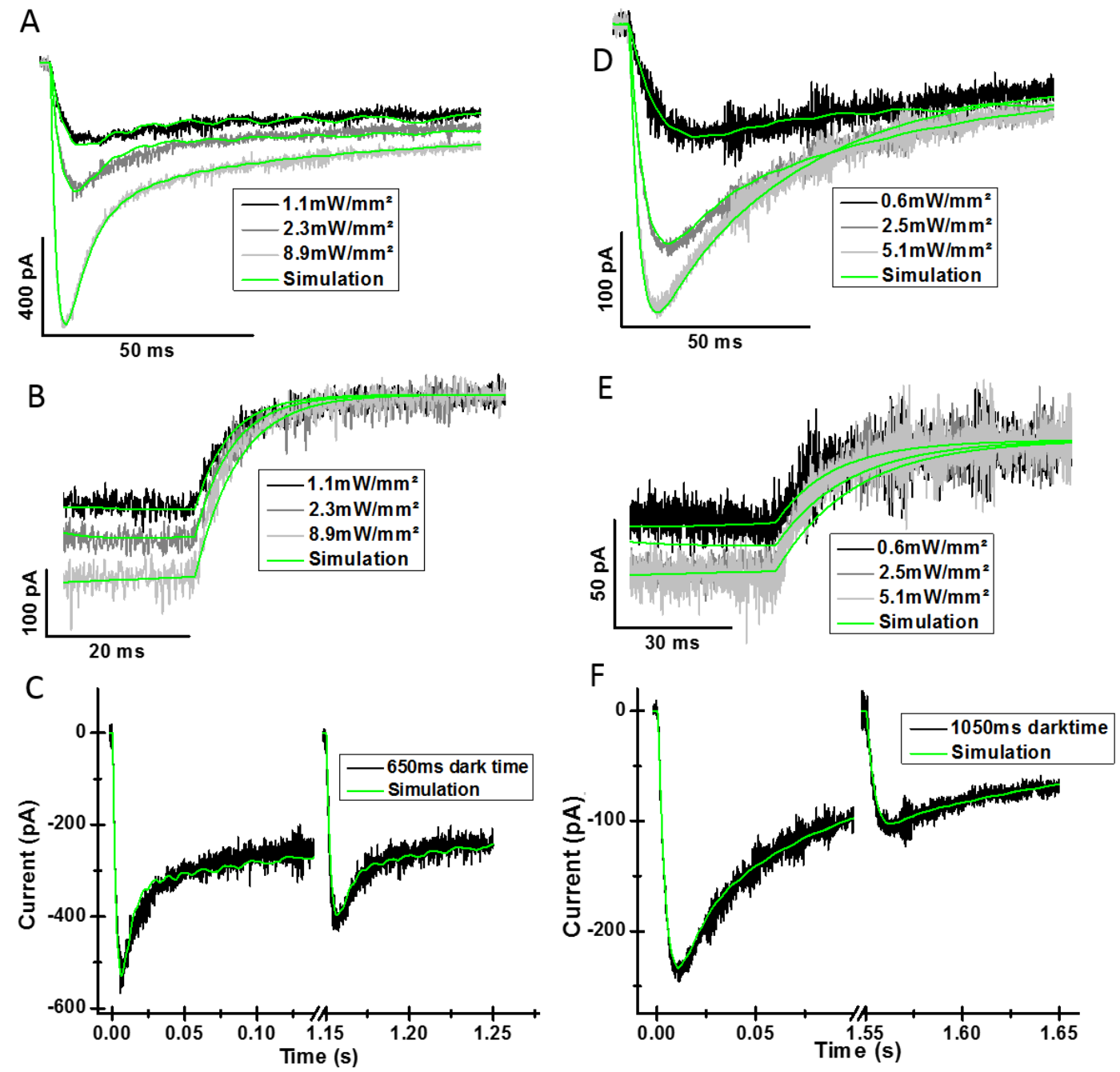

Figure 5.8: Optimized traces of Chronos/ChR2. The recorded current traces (gray) are overlaid with the simulated curves (green). A: Onset of the current response of Chronos to square light pulses of different intensities. B: Deactivation of Chronos after square light pulse. C: Response to two square pulses separated by $650 \mathrm{~ms}$ of darkness. Shown in green is the simulated data from the complete model of Figure 5.7 with parameters optimized to represent the global data. All simulated curves presented were calculated using the recorded light waveform as stimulation and use the same global parameters. D-F: The corresponding data for a cell transfected with ChR2, along with the simulated curves. 


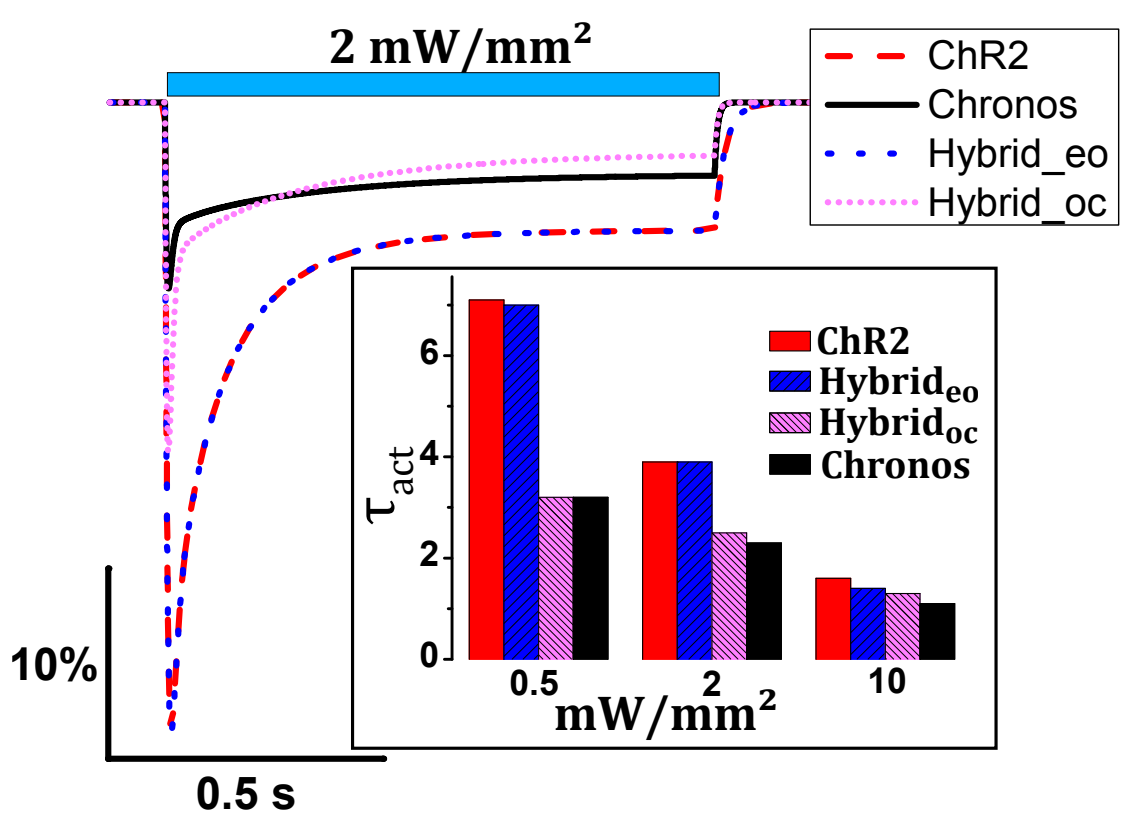

Figure 5.9: Hybrid Evaluation. Simulated traces for different ChR2/Chronos hybrid parameter sets (as given in Table 5.1) to a $1 \mathrm{~s}$ square pulse with $2 \mathrm{~mW} / \mathrm{mm}^{2}$. Except for a greater time lag after the stimulus onset, the pure ChR2 trace (red dashed) is barely distinguishable from the hybrid having the $k_{e o}$ parameters from Chronos (blue dotted). The hybrid with Chronos' $k_{o c}$ values (pink dotted) shows a faster on and off rate as well as a strongly reduced overall current. The trance for Chronos is presented as a reference in black. All curves are normalized to the current obtained if all molecule were in the open state with the highest conductance. The blue bar indicates the duration of the light pulse. Inset: Activation times derived from simulations of ChR2 (red), Chronos (black) and the two hybrids at different intensities. Exchanging of $k_{o c}$ leads to nearly the same speed as Chronos for 0.5 and $2 \mathrm{~mW} / \mathrm{mm}^{2}$, while $k_{e o}$ seems to only have a comparable influence at high intensities. Activation time is defined here by an exponential fit to the simulated initial current rise during illumination with a square pulse. 


\begin{tabular}{|l||l|l|l|l|l|l|l|l|l|l|}
\hline & $\alpha$ & $k_{e o}$ & $k_{o c}$ & $k_{c g}$ & $\alpha^{l}$ & $k_{o c}^{l}$ & $k_{c g}^{l}$ & $k_{\text {adapt }}$ & $k_{\text {relax }}$ & $k_{\text {rtg }}$ \\
\hline \hline & $\frac{m m^{2}}{m W s}$ & $1 / \mathrm{s}$ & $1 / \mathrm{s}$ & $1 / \mathrm{s}$ & $\frac{\mathrm{mm}^{2}}{\mathrm{mWs}}$ & $1 / \mathrm{s}$ & $1 / \mathrm{s}$ & $\frac{\mathrm{mm}^{2}}{\mathrm{mWs}}$ & $1 / \mathrm{s}$ & $1 / \mathrm{s}$ \\
\hline ChR2 & 73 & 2350 & 86 & 43 & 1.8 & 48 & 7.2 & 16 & 1.6 & 0.29 \\
\hline Chronos & 71 & 5360 & 241 & 78 & 3.5 & 161 & 12 & 7.6 & 1.3 & 0.98 \\
\hline Hybrid $k_{\text {eo }}$ & 73 & 5360 & 86 & 43 & 1.8 & 48 & 7.2 & 16 & 1.6 & 0.29 \\
\hline Hybrid $k_{o c}$ & 73 & 2350 & 241 & 43 & 1.8 & 161 & 7.2 & 16 & 1.6 & 0.29 \\
\hline
\end{tabular}

Table 5.1: Parameters of the Markov-model given in Figure 5.7 after optimization for Chronos and ChR2 along with the hybrids created from the Parameters. The ' 1 ' following some parameters indicates that this value is of the light adapted cycle.

current waveform of the $k_{e o}$ hybrid. The effect of the $k_{o c}$ hybrid on the other hand is quiet dramatic. As expected, the decay speed of the current after the illumination ends is faster, as the conducting states have shorter lifetimes. In addition, the risetime of the current after illumination onset is also faster, since the hybrid reaches the point in time at which more molecules leave the conducting state than enter it more quickly. The cost is a considerable decrease in the overall current, as a larger fraction of the molecules spend time in one of the non-conducting states during illumination. This is a direct consequence of increasing the speed of the channel by decreasing the lifetime of the conducting states.

However, during the experiments of this study, the overall current of cells expressing Chronos was high. There are three possible explanations for these large currents in Chronos. One possibility is a larger number of functional molecules in the membrane of cells transfected with Chronos, the second is a higher conductivity of each single Chronos channels as compared to ChR2 and the last is a decrease in the lifetimes of the non-conducting states. The last explanation would result in a generally faster light cycle in Chronos, so that molecules would not gather in non-conducting states. Our simulations show, that even though the last point seems to be true in Chronos (see Table 5.1), the open probability is still between $20 \%$ and $50 \%$ of ChR2's.

\subsubsection{Voltage dependence of Chronos}

Aside from the possibility of using simulations to evaluate the properties of the molecular transitions, they are also useful for predicting the currents to certain stimuli in experiments. This way, optimized stimulation protocols can be developed without extensive experimental trials. In the study of the AIS as described in section 4.4 such simulations become important for example, as light adaptation of the ChR decreases the ability of the light stimulation to depolarize the cell. This needs to be compensated for in the protocols for driving illumination. Simulations can also be used to calculate currents in experiments where it is impractical to record the actual currents experimentally [9]. The model parameters in this study are all derived for cells clamped to a certain voltage however. This adds another potentially complicated factor for usage in non voltage-clamped cells. To estimate the influence of changes in the voltage, $1 \mathrm{~s}$ light pulses of a given intensity were pre- 
sented to cells clamped at different voltages and key parameters of the traces were evaluated. Figure 5.10 (A) shows the onset of the current response to a light step at different voltages. The traces are fitted by exponential functions with one global time constant to see if this parameter changes drastically at different voltages. In our experiments this was not the case. Instead the main difference during the onset is a change in the amplitude of the current. Due to the large difference between peak and steady-state current, both were investigated separately to determine if peak and steady-state current change was similar at different voltages. Figure 5.10.(B) shows the change in amplitude for the peak and the steady-state current after normalization to the largest value. The peak and steady-state amplitudes are changing in a very similar fashion, indicating that a simple scaling of the currents leads to a good approximation of the effect induced by a change in voltage. Ramping the voltage after reaching the steady state during a long light pulse gives a direct measurement of the scaling relationship. The resulting current/voltage curve is given in Figure 5.10 (C) for both Chronos and ChR2. It is similar to curves previously published for ChR2 [47]. Using this I/V curve, the simulated currents can be scaled from their initial value optimized at $-60 \mathrm{mV}$ to the proper voltage at each time point either during or even after simulation. It should be noted however, that the deactivation kinetics occurring during the dark right after illumination do possess some voltage dependence as was already reported for ChR2 [78, 85], possibly due to different ion permeability of the open states. While the contribution of the current changes during the deactivation usually does not dominate the overall current in Chronos due to its fast decay, this could have some influence during experiments with short enough pulses. A weak voltage dependence in the activation and a stronger for deactivation differs from the findings in [47, where both activation and deactivation have a mild voltage dependence. However this could be due to different ion gradients across the membrane, as for Williams et al. the deactivation was also always well described by a single exponential decay, while it gained bi-exponential character for higher voltages in our experiments.

\subsubsection{High-intensity deactivation}

Another important factor worth noting is the reduction of current after high-intensity illumination as shown in Figure 5.12 for a cell expressing Chronos. Here two long weak pulses of the same intensity are presented with a long pulse of high intensity in between. During the first weak pulse a stable steady state is reached (aside from slow fluctuation due to thermal fluctuations of the diode laser) while during the strong pulse the current steadily declines. The reduction of the current seen during the high-intensity pulse is still present during the second weak pulse shortly after. This indicates a real reduction of the steady state during the high intensity pulse and not a slow transition to equilibrium. Although some recovery does take place, the steady state does not reach its initial value during the seconds of probing with a weak pulse after strong illumination. Repeating the experiment after a dark period of $40 \mathrm{~s}$ yields a similar trace, but with a generally reduced current, showing that even after 40 seconds in the dark no complete recovery has taken place. The inset shows 


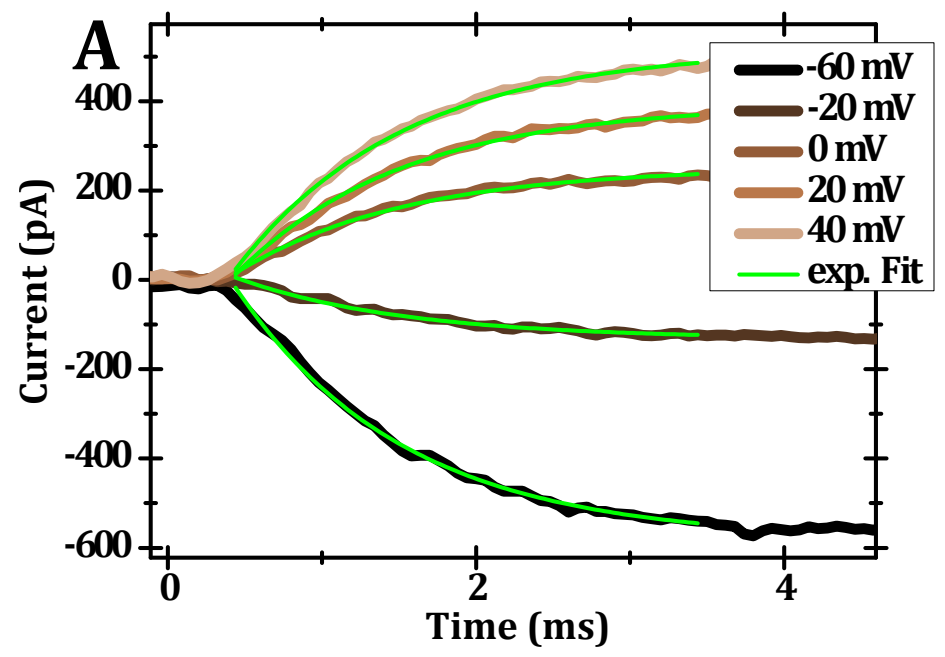

B
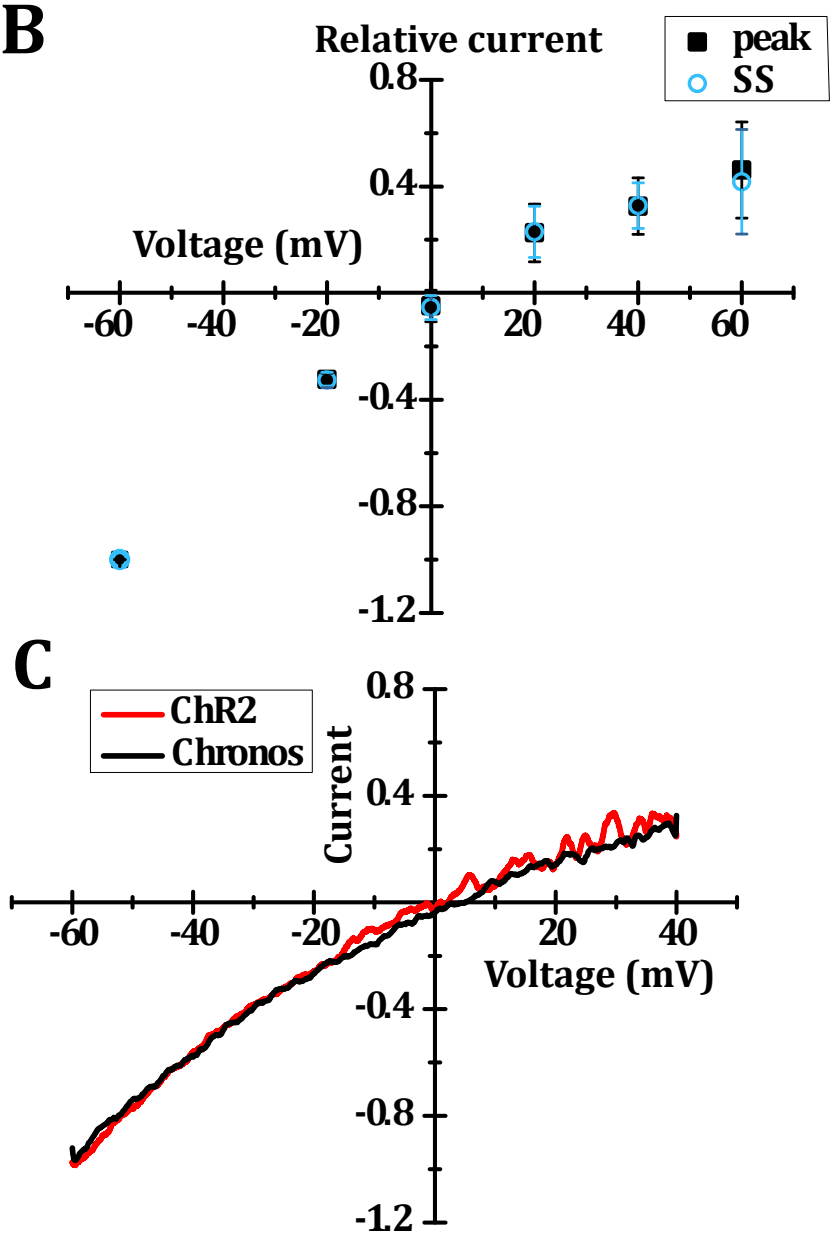

Figure 5.10: Voltage dependencies. A: Current rise of Chronos at different clamped voltages (brown) fitted with exponential functions (green) using one global time constant. B: Normalized peak (square) and steady-state (circle) values at different voltages for Chronos $(n=11)$. The relative change of the current for steady state and peak does not differ significantly after normalizing the values to the highest absolute current. This indicates the possibility of treating the influence of the voltage in simulations by simple scaling of the current according to a current/voltage curve as shown on panel $\mathbf{C}$ for Chronos (black) and ChR2 (red). 
the first pulse of three such experiments with a $40 \mathrm{~s}$ dark period in between. This effect is fundamentally different from the light adaptation in Figure 5.5 as it also reduces the steady-state current and lasts longer than the reduction caused by light adaptation. As the steepness of the current loss increases with higher intensities and the trace is of an exponential shape, the data was compared to a pure exponential decay with a time constant proportional to the intensity as would be the case for an absorbing transition leading to a permanently non-conducting state (or a destroyed molecule). A comparison in several transfected cells indicates that this is not the case.

Instead it was assumed that the molecules in the light cycle are transferred into a non-conducting state via absorption of a photon and recover back via relaxation with the time constant $\tau_{\text {recover }}$ as shown in Figure 5.11.

\section{Inactivated state}

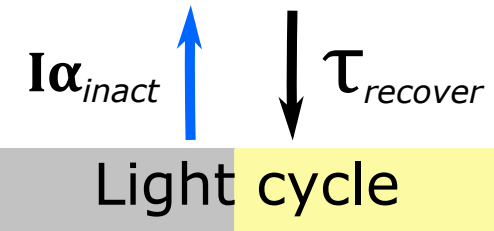

Figure 5.11: Bleach model. An inactivated nonconducting state is reached by absorption and slowly relaxes back with the time constant $\tau_{\text {recover }}$.

Since all the molecules have to be either in the light cycle $L_{c}$ or in the inactivated state $I_{s}$ the differential equations are symmetric and become relatively simple:

$$
\frac{d L_{c}}{d t}(t)=-\frac{d I_{s}}{d t}(t)=-I \alpha_{\text {inact }} L_{c}(t)+\frac{I_{s}(t)}{\tau_{\text {recover }}}=-I \alpha_{\text {inact }} L_{c}(t)+\frac{L_{c}(0)-L_{c}(t)}{\tau_{\text {recover }}}
$$

This equation is solved by a single exponential function decaying to a steady state determined by the absorption $I \alpha_{\text {inact }}$ and the recovery time-constant $\tau_{\text {recover }}$.

$$
L_{c}(t)=\frac{L_{C}(0)}{1+I \alpha_{\text {inact }} \tau_{\text {recover }}}\left(I \alpha_{\text {inact }} \tau_{\text {recover }} e^{\left(-\left(I \alpha_{\text {inact }}+1 / \tau_{\text {recover }}\right) t\right)}+1\right)
$$

The assumption that an absorption leads to a slowly recovering non-conducting state leads to a better representation of the data and an inactivation constant of $9 \pm 5 \mathrm{~mm}^{2} / \mathrm{mWs}$ (standard error of mean). This would mean an initial reduction of $\sim 45 \%$ right after a 10 second stimulation with $10 \mathrm{~mW} / \mathrm{mm}^{2}$. However, the effect is not perfectly explained by a fully recovering dark state. This could be because some of the reduction is irreversible or some of its light dependence is not linear. However, even without exact characterization of the effect it becomes obvious that higher intensities cause weaker depolarization at later times and thus should be avoided if possible. The reduction already becomes apparent for intensities of around $10 \mathrm{~mW} / \mathrm{mm}^{2}$, which are often reached during experiments using Channelrhodopsins [96, 97.

\subsubsection{Comparison to spectroscopy and other models}

To my knowledge, the literature model yielding the closest representation to that of this study, is found in [90]. A graphical representation of this model is given in 


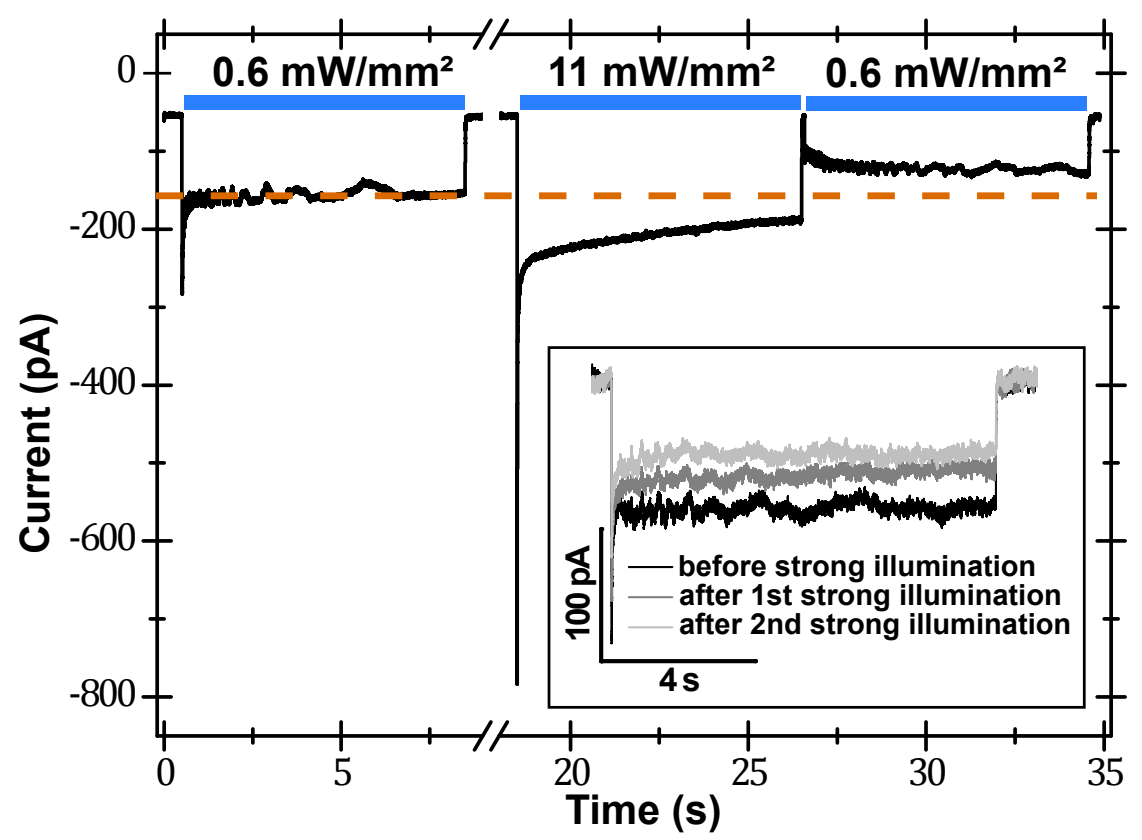

Figure 5.12: Bleaching. The current response to two long identical pulses with a high-intensity pulse in between for Chronos. The orange dotted line indicates the steady-state current before strong illumination. The strong pulse not only causes the peak reduction in the second pulse, visible during the reversible light adaptation shown in Figure 5.5, but also reduces the steady state current which does not recover fully in the recorded time. The inset shows the response to the first pulse of a similar stimulation protocol repeating the stimulation three times with a $40 \mathrm{~s}$ dark time in between each trial. Even in those $40 \mathrm{~s}$ the current does not return to its initial value. 
Figure 5.13 (A). Mapping the corresponding spectroscopic states to our electrophysiological states leads to $E \leftrightarrow P 390, O \leftrightarrow P 520, C_{d} / C_{l} \leftrightarrow P 470 / P 480$ and $G_{d} / G_{l} \leftrightarrow$ $D 470 / D e s 480$. For the spectroscopically measurable transitions, a more quantitative comparison is possible by using the lifetimes of the spectroscopic states of ChR2 as given by [86]. In the spectroscopic measurement, the transition to the open state P520 possesses a time constant of $1.5 \mathrm{~ms}$, which is far greater than in our model. However, this would lead to a delay in the conductance onset not seen in our experiments, indicating that this transition is faster in physiological conditions, possibly due to a faster deprotonation of the Schiff base. This idea seems to be reinforced by other electrophysiological studies stating that transitions to the open state have to be faster than $1 \mathrm{~ms} \mathrm{[78]}$ and also by spectroscopic measurements estimating the transition to be $0.15 \mathrm{~ms}$ [85]. The decay of the open state is considered to be voltage dependent [85, 78] and has a time constant of about $10 \mathrm{~ms}$ at $0 \mathrm{~V}$ [85, 86, 78], in agreement with our finding. The relaxation from the light adapted state is also a voltage and $\mathrm{pH}$ dependent process [78] explaining the extremely long time constant of the Des480 $\rightarrow$ D470 transition of $\sim 40$ s reported in [86]. In accordance with the faster relaxation we observed in Figure 5.6 this transition is far faster in our model. As already stated, the fast kinetics of Chronos seem to stem from its short-lived conducting state. In our model this would be an analogue to the spectroscopic P520 state in ChR2. A process closely related to the conducting states decay is the bonding of E90 located in the pore of the channel [86, 98]. The central gate of ChR2 (shown in Figure 5.13 (B)) consisting of S63, E90 and N258 [99, 100] is conserved in Chronos except for the serine which is exchanged for the non-polar alanine. The faster decay of the open state could therefore lie in a more accessible E90 in Chronos. This might also be connected to a change in the conductance of $\mathrm{H}^{+}$ as compared to ChR2, which could be another factor in the large currents observed in spite of the fast decaying open state.

Interestingly, there is also substitution of methionine for E123, an amino acid conserved in many channelrhodopsin variants and whose mutation lead to the engineered fast ChR2 derivatives termed ChETA [81]. As E123 influences the stabilization of the Schiff base, this might be partially responsible for the fast transition into the conducting state observed in this study. 

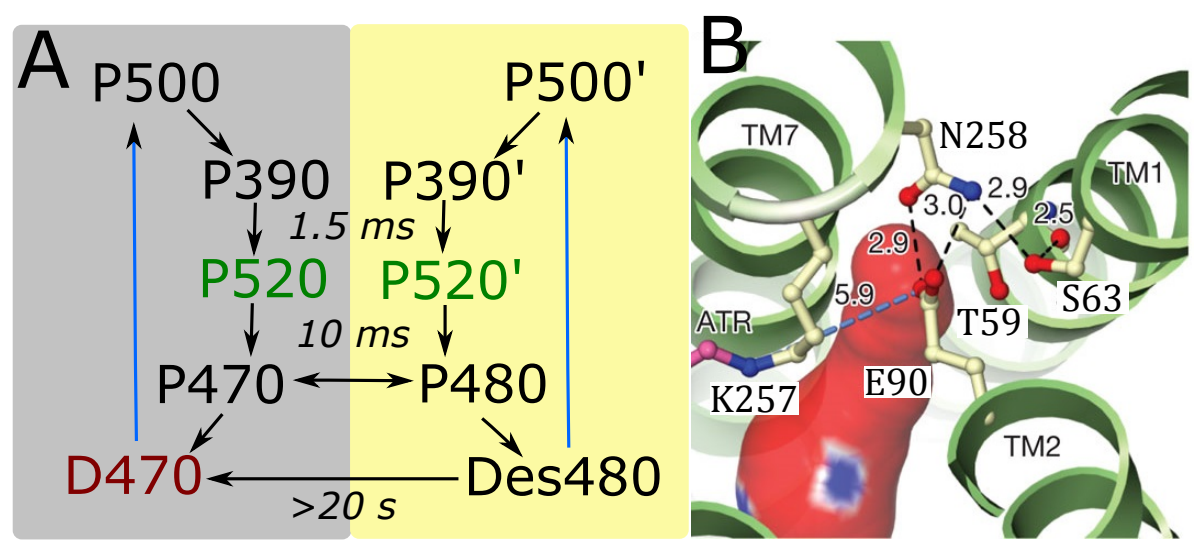

Figure 5.13: Comparison to other models. A: Model from the literature showing the closest resemblance to that used in this study (Figure 5.7). Adapted from [90]. Time constants from [86] are inserted. B: Structure of a chimaera between ChR2 and ChR1 at the central gate. The numbering of the amino acids has been changed to represent that of ChR2 as suggested by the author. The red and blue structure represents the walls of the pore in the closed conformation shown. Part of the all-trans retinal is shown in pink. Adapted from [99].

\subsection{Discussion}

Rational design of optimal optogenetic tools requires a deep understanding of the biophysical processes during the gating of channelrhodopsins. An electrophysiological model that aims at the elucidation of actual molecular parameters and thereby gives the possibility to create a closer link to spectroscopy is an important step in this direction.

For our experiments we chose the most widely used channelrhodopsin ChR2 [93] and the newly discovered Chronos reported to possess faster kinetics than any other channelrhodopsin [84]. This study confirmed the fast kinetics of Chronos. The construct used also provided a high attainable current showing that Chronos is capable to extending the applicable range of channelrhodopsins to stimulation at higher frequencies than was previously possible. Chronos also exhibits light adaptation not only weaker than ChR2, but also recovers faster into the fully dark adapted state. In a first step, the analysis was restricted to stimulation protocols isolating a small number of parameters in minimal models representing only parts of the light cycle. Then an extended branching model aimed at replicating all the phenomena seen without disconnecting from an actual molecular state representation was introduced. The parameters of this model were tightly constrained by employing an optimization routine making use of the complete shape of the response waveform combined with stimulation covering a wide range of intensities and timescales.

The complete model used consists of two branches with four states each. These four states are connected in an identical circular manner for both branches which only differ in the transition rates. The branch including the ground state represents the dark adapted loop and possesses a transition to the other branch via its open state. Molecules occupying states in the second loop are light adapted. All the transitions 
in the model either possess a fixed rate-constant or a linear light-power dependence as expected from a single photon process.

The model describes both channels reasonably well, enabling a direct comparison of the kinetics in terms of the underlying parameters. Simulations with hybrid parameter sets identified the fast decay of the conducting state to be mostly responsible for the superior speed of Chronos. These simulations also estimate a decreased open probability of Chronos compared to ChR2 of at least a factor of two although good expression levels and possibly higher conductivity seem to counter this effect. 


\section{Experimental/theoretical procedures}

\subsection{Materials and Methods}

\subsubsection{Pipettes}

\section{Pulling pipettes}

The pipettes harboring the silver/silverchloride electrodes for the SICM were fabricated from borosilicate glass tubes (Science Products, Hofheim, Germany) fitted with filaments along the inside of the tube for easier filling. The glass tubes had an outer and inner diameter of $1 \mathrm{~mm}$ and $0.58 \mathrm{~mm}$ respectively and were pulled into micro pipettes using a P-97 Flaming/Brown micro-pipette puller (Sutter Instrument, Novato, CA). By inducing large currents through a metal ring surrounding the glass tube, high enough temperatures were created to deform the glass and pull its ends apart. By using several heating cycles accompanied by well defined pulling forces, pipettes of different geometries could be created. The pipettes created for whole cell experiments had resistances between 1 and $5 \mathrm{M} \Omega$ in the ionic bath solution. Pipettes used for high resolution SICM imaging had resistances between 60 and $200 \mathrm{M} \Omega$ and tip opening diameters between 60 and $150 \mathrm{~nm}$.

\section{Determining pipettes properties}

In electrophysiology the size of the pipette used for recording is usually described by the electrical resistance, as this value gives important insight into the capability of the electrode to inject currents. (see Section 2.2) The physical size of the pipette opening usually only plays a role, if the examined area should be limited, as in single channel recording. For the SICM technique the size of the opening is of much greater importance however, as it not only limits the resolution of the image, but also determines the exact distance from the sample at which a certain current decrease takes place. For perfectly cone shaped pipette tips the resistance can be analytically calculated in the following way:

$$
R_{P}=\int_{l_{0}}^{l+l_{0}} \frac{d x}{A}=\int_{l_{0}}^{l+l_{0}} \frac{d x}{\pi(x \tan (\alpha))^{2}}=\frac{\rho}{\pi \tan ^{2}(\alpha)}\left(\frac{1}{l_{0}}-\frac{1}{l+l_{0}}\right)
$$

with $l$ being the distance between the pipette opening and the silver/silver-chloride electrode, $l_{0}$ the distance between a theoretical complete cone tip and the actual 
opening while $\alpha$ is the cone angle as defined in Figure 6.1. The tip opening radius $r_{i n}$ fulfills the relation $l_{0}=r_{i n} / \tan (\alpha)$ which leads to

$$
R_{P}=\frac{\rho}{\pi \tan ^{2}(\alpha)}\left(\frac{\tan (\alpha)}{r_{i n}}-\frac{1}{l+r_{i n} / \tan (\alpha)}\right) .
$$

Assuming a small angle and thus $\tan (\alpha) \approx \sin (\alpha) \approx r_{\text {electrode }} / l$ with $r_{\text {electrode }}$ being the radius of the pipette at the electrode the equation 6.1 .2 becomes

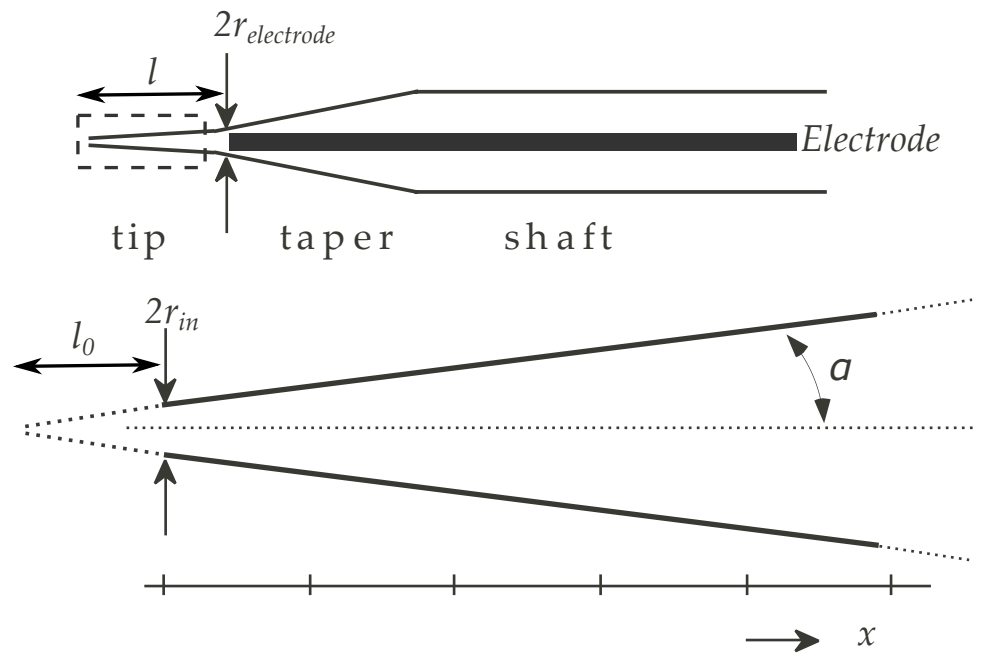

Figure 6.1: Pipette resistance. For a perfectly conically shaped tip, the resistance of a patch pipette can be calculated analytically. A: Schematic of pipette with inserted electrode. B: Enlarged version of dashed box. As the total resistance is dominated by the tip, integration only has to be performed from $x=l_{0}$ to $x=l$. Adapted from [101].

$$
R_{P}=\frac{\rho l}{\pi r_{\text {in }}\left(r_{\text {in }}+r_{\text {electrode }}\right)} .
$$

Under the additional assumption that $r_{\text {electrode }} \gg r_{i n}$ the equation is simplified to

$$
R_{P}=\frac{\rho l}{\pi r_{\text {in }} r_{\text {electrode }}},
$$

as is found in the literature 23, 24]. The assumption of perfectly cone shaped pipette tips is highly idealized however, and especially for pipettes pulled in multiple pulling cycles the actual shape is considerably more curved. The pipette resistance alone is thus a bad indicator for the size of the opening, and additional information is needed. Determining the actual size of the opening becomes difficult for scales of the same size or smaller than the wavelength of visible light, as optical examination can no longer resolve the pipette tips as was shown by Sebastian Schwarz, one of our diploma students. Advanced methods such as electron microscopy can easily determine the dimensions of such pipettes and were utilized to cross check our methods (Figure 6.2). Electron microscopy requires a conductive coating of the glass pipettes however, making them unusable for electrophysiological measurements 
afterwards. This drawback combined with the complexity of the measurement makes it impractical to use during the process of optimizing the pipette shape using the micro-pipette puller. Instead another feature of the small pipette opening can be used to determine its size: the force needed to produce bubbles from the pipette tip in a liquid [102, 103]. Assuming a perfectly round opening, the relationship between the inner radius $r_{i n}$ and the pressure $P_{b u b b l e}$ at which bubbles are able to leave the pipette is

$$
r_{i n}=\frac{2 \sigma}{P_{b u b b l e}} .
$$

with $\sigma$ being the surface tension of the liquid. To determine $P_{b u b b l e}$, the pipette was fitted into an air-tight holder, which in turn was attached to high pressure medical tubing and a three-way valve (Medtron, Saarbrücken,Germany). The pipette was then immersed in methanol, as the low surface tension reduced the required pressure for bubble extraction. The glass holding the methanol was illuminated by a bright light source at an appropriate angle to optimize the visibility of bubbles. Pressure was applied by compressing a syringe attached to the valve and the pressure was measured by a digital manometer (PCE Instruments, Southampton, UK ) for large pipettes. For pipette radii of less than $80 \mathrm{~nm}$ the pressure was estimated by the compression of the volume, as the required pressures exceeded the range of the available manometer.

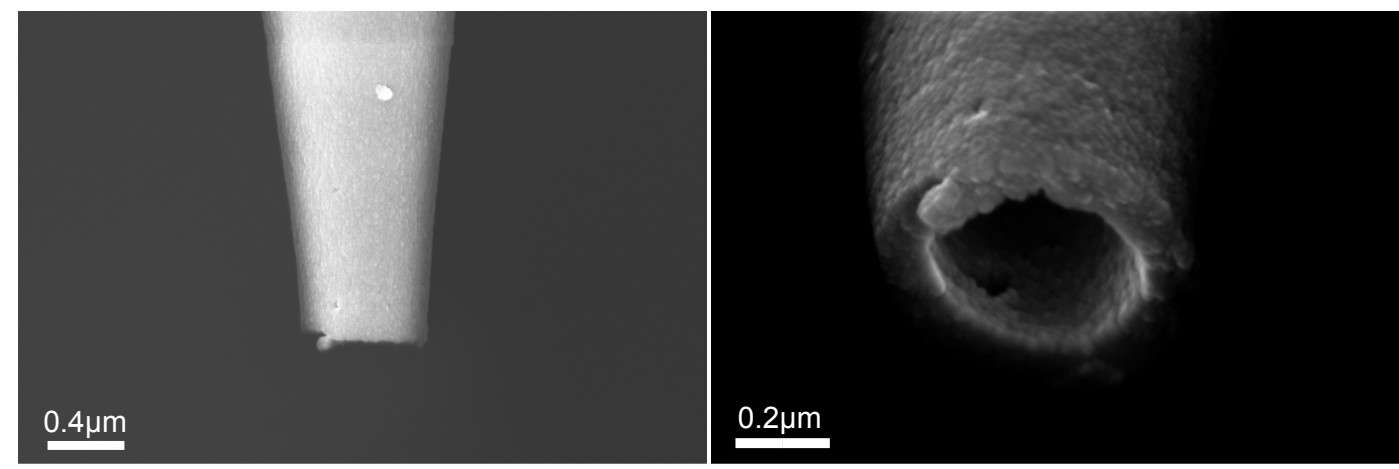

Figure 6.2: EM image of pipette. Electron microscopy is able to resolve the opening of a micropipette easily. However, the procedure is time consuming, and due to the conductive coating needed for electron microscopy the pipettes are not usable for electrophysiological experiments after imaging. Images were taken by Sebastian Schwarz.

The bubble pressure technique is a fast and non destructive method for investigating the pipette tip size. To relate the opening size to the bubble pressure via equation 6.1.5 requires the assumption that the opening is well approximated by a circle, and that the end surface is reasonably flat. For pipette tips with openings of $100 \mathrm{~nm}$ and less, flaws in the tip quality are not optically discernible. Some information on the tip quality can be derived from the access resistance of the pipette 
however. For a pipette tip in solution and far from any surface, the access resistance is simply a constant resistance in series with the pipette resistance approximated by equation 6.1.2. The value of the access resistance infinitely far from a surface can be calculated by using the assumption that the tip opening acts like a disk shaped electrode. The resistance $R$ between electrodes in conducting media and the capacitance $C$ between conductors in isolating media are related by a simple transformation, since the field lines (and thus the current flow in ionic solution) are the same [104]. The relation is

$$
R=\frac{\epsilon \rho}{C}
$$

where $\rho$ is the resistivity of the conducting medium and $\epsilon$ is the permittivity of the isolator. Solving the capacitance of a conducting disk and a sphere infinitely far away leads to

$$
C=8 \epsilon r_{i n},
$$

with $r_{i n}$ being the radius of the disc. As the access resistance is determined by only one half space, half of this value needs to be used so that the resistance becomes

$$
R_{\text {access }}=\frac{\rho}{4 r_{i n}} .
$$

Since in real experiment the reference electrode has a finite size and is not infinitely far away, this value is only an approximation. Experimental examinations have yielded a slightly lower value of $R_{\text {access }}=\rho / 5 r_{i n}$ for the access resistance of real pipettes [20].

When approaching a sample, the access resistance rises dramatically as explained in section 3.1. Exchanging the built-in amplifier of the SICM with an external patchclamp amplifier gave us the ability to record the relationship between the current and the tip/sample distance as already shown in Figure 3.1 (B). For pipettes possessing uneven tips the maximal current reduction achievable by approaching a flat sample is reduced and the shape of the approach curve is distorted. As most SICM samples reside on glass, a smooth enough surface is present for evaluating the quality of the pipette tip before the actual measurement. Figure 6.3 gives an example of pipettes with different tip openings and resistances. As can be seen, both the minimal current and the steepness of the reduction vary. From the inability of the $27 \mathrm{M} \Omega$ curve to reach very large reductions, it can be deduced, that the pipette tip is not perfectly flat. Furthermore, the distance at which the access resistance starts to noticeably increase depends on the tip size opening. For smaller tips, the reduction takes place at smaller distances from the sample then for larger tips. (It could also be said that larger tips sense the surface earlier than small tips.) The distance between the start of current reduction and contact on the glass surface is plotted against the pipette radius as determined by the bubble technique in Figure 6.3 (B). A clear relation is seen, even for pipettes with bad quality tips. Interestingly, the factor for the linear approximation is nearly exactly ' 1 '. The distance between the pipette and the sample during a SICM scan is therefore close to the pipette opening radius. 


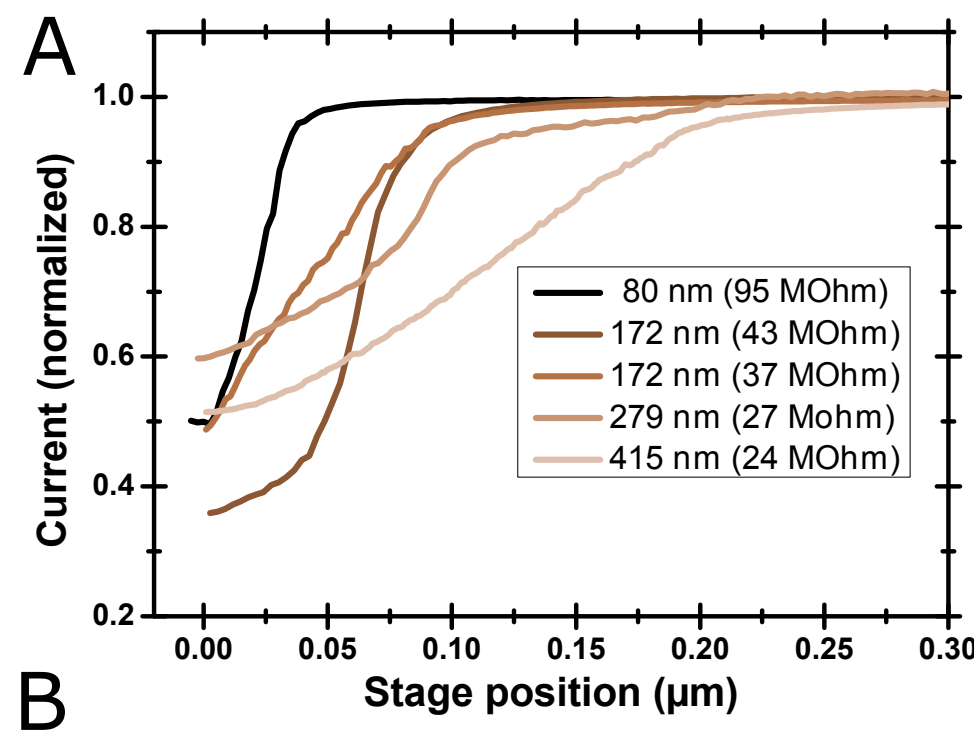

Figure 6.3: Approach curve analysis. A: Approach curves for different pipettes. The zero position point is defined as the point of minimal current. B: The distance between the point of reduction onset and the contact is plotted against the pipette

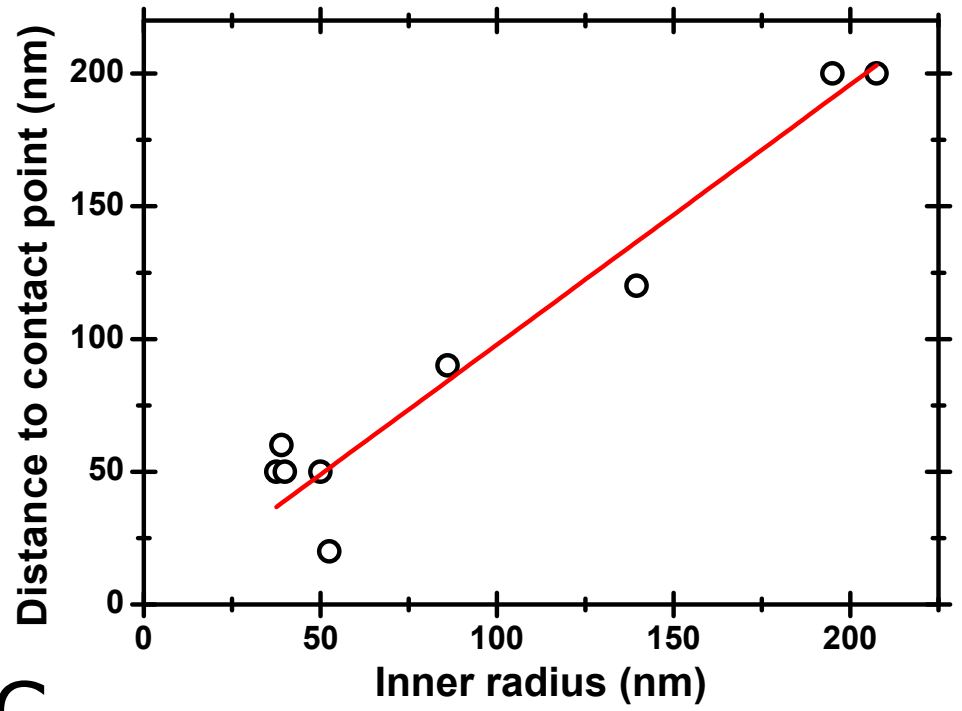
opening as measured via the bubble technique. The red line shows a linear fit to the data. The constant of proportionality is nearly one. C: Example for a strongly distorted approach curve showing two phases. Inset: A possible geometry leading to a two phase curve. During the first part of the approach the access resistance on all sides increases (reduced access indicated in red). Once the pipette bends due to contact with the surface, the access resistance on one side of the pipette becomes smaller (blue indicated increasing access) while on the other side it continues to increase. 
By evaluating the approach curve it is possible to create pulling protocols for the pipette puller which produce pipettes possessing high tip quality with high reproducibility. They are also useful for determining the tip quality after accidental or deliberate damage to the pipette. I found two distinct ways of deliberately removing the topmost tip of the pipette which I term 'snapping' and 'breaking'. Snapping is usually only possible for thin pipettes with a small opening angle $\alpha$ as defined in Figure 6.1. These pipettes can be bent at the glass surface as can even be seen optically for large displacements past the contact point. For small displacements this can still be observed in the approach curve as a reversible rise in the current after contact, as the opening of the tip bends away from the surface. By using fast collisions with the surface and low displacements past the contact point it is possible to remove part of the tip while retaining a high quality tip, possibly by snapping off the tip while in a strongly bend position. During a snapping event the resistance of the tip will usually not decrease by more that a factor of 0.5 . Deliberate snapping of the tip can be useful during long measurements such as the investigation of the midbody dynamics shown in Figure 3.4. During such long measurements the pipette opening can become obstructed by particles in the solution and exchanging the pipette is sometimes impractical. An additional reason for breaking the pipette is the desire to reduce the resistance of the electrode for a secondary measurement after image acquisition as described in section 4.4. However, for wider and stiffer pipettes the quality of the tip after breaking is often strongly reduced. An especially notable example is shown in Figure 6.3 (C). Here the pipette not only fails to reach a large reduction of current but also displays two distinct phases in its approach curve. Figure 6.3 (inset) gives a possible explanation for this behavior.

\subsubsection{Setup design}

\section{Setup SICM}

Two different SICM models constructed by 'Ionscope' were used in the course of this thesis. Most of the work was performed using the ICnano SH01 (Ionscope Ltd, Melbourn, United Kingdom). The main part of the SICM consists of three servo and piezo stages (Physik Instrumente (PI) GmbH \& Co. KG, Karlsruhe, Germany) for movement in all three dimensions. The sample holder is mounted on a wide range $2 \mathrm{D}$ servo motor stage allowing coarse movement across an entire petri dish. The fine lateral movement during scanning is performed by a $2 \mathrm{D}$ piezo stage providing a range of $100 \mu \mathrm{m}$. The pipette holder is connected to another servo stage for immersion and retraction of the pipette from the sample and is mounted to a fast piezo stage spanning $25 \mu \mathrm{m}$ for height adjustment during scanning. To provide a low-noise environment, sample holder, pipette and headstage of the patch-clamp amplifier were enclosed in a Faraday cage. This cage and its internal parts are referred to as the SICM 'scan head' in the following. The servo and piezo motors were driven by an external driver (Physik Instrumente). To provide a low-noise signal and to enhance the flexibility of the setup, the built-in amplifier of the SICM controller was exchanged for an external EPC10 amplifier (HEKA, Lambrecht, Germany). The signal recorded by the patch pipette was then fed to the SICM controller (IC- 
nano), which performed the fast real-time feedback response necessary for keeping the pipette from colliding with the sample during scanning. Both the EPC10 and the SICM controller are controlled by a personal computer. 'Patchmaster' (HEKA, Lambrecht, Germany) was used to control the voltage at the pipette and extract all electrophysiological data. The program 'ScanIC Control' was used as interface for the SICM controller and to record SICM topography images. The SICM scan head is mounted on an inverted microscope (Eclipse-Ti, Nikon, Japan) so that transparent samples can be investigated using bright-field and fluorescent microscopy in addition to SICM. A special holder for commercially available multi electrode arrays (MEAs) was constructed by our mechanical and electronic workshops. A general overview of the essential SICM parts is given in Figure 6.4.

Additionally, a second setup was recently added based on a SICM prototype called ICnano2043. This version differs from the SH01 in some key factors important for our investigations. One of the most important is the different approach to scanning the sample. Instead of moving the sample underneath the SICM pipette, the pipette itself is tilted by a set of three piezo crystals, reaching a lateral scan area of $50 \times 50 \mu \mathrm{m}$ and a height difference of $12 \mu \mathrm{m}$. Due to this approach, the sample does not move relative to the surrounding base plate of the SICM. In combination with not having a non-removable Faraday cage, this makes it possible to easily add a second recording pipette, which in this case was mounted on a Sensapex SMX micro manipulator (Sensapex, Oulo, Finland) attached to the baseplate of the microscope. To scan samples of greater height than $12 \mu \mathrm{m}$ a second piezo stage for z-positioning is attached to the pipette granting another $100 \mu \mathrm{m}$ of displacement. The prototype is driven by a controller of the type 'Icnano2000' which in turn is controlled by a LabVIEW (National Instruments Corporation, Austin, TX) based software called 'ICnano2000 Control application' (Ionscope Ltd, Melbourn, United Kingdom). The scan head was mounted on an inverted microscope (DMI3000B, Leica Microsystems, Wetzlar, Germany). Heating of samples is possible thanks to a custom holder created by our mechanical workshop as shown in Figure 6.5 (B). The brass metal ring holding the petri dish is heated using a 'TC200 Temperature controller' (Thorlabs, Newton, NJ).

Both SICM systems were mounted on an optical table (Newport Corporation, Irvine, CA) with pneumatic vibration dampers for improved mechanical stability.

\section{Extra cell setup}

For patch-clamp measurements and SICM imaging a EPC10 by HEKA was used. This high-sensitivity amplifier consists of two parts. The first one is the main unit, which contains the power supply, the signal processing electronics, the $\mathrm{A} / \mathrm{D}$ and $\mathrm{D} / \mathrm{A}$ converters and the connectors for analog and digital input/output. The second part is the headstage incorporating circuits similar to those shown in Figure 2.4. This small preamplifier can be placed close to the recording electrode, ideally inside the same Faraday cage to minimize noise pickup. The headstage incorporates three voltage clamp circuits with different feedback resistors. The desired circuit can be selected remotely via the PC interface, making it possible to provide high signal-tonoise ratios when recording large currents as in whole-cell measurements or small 


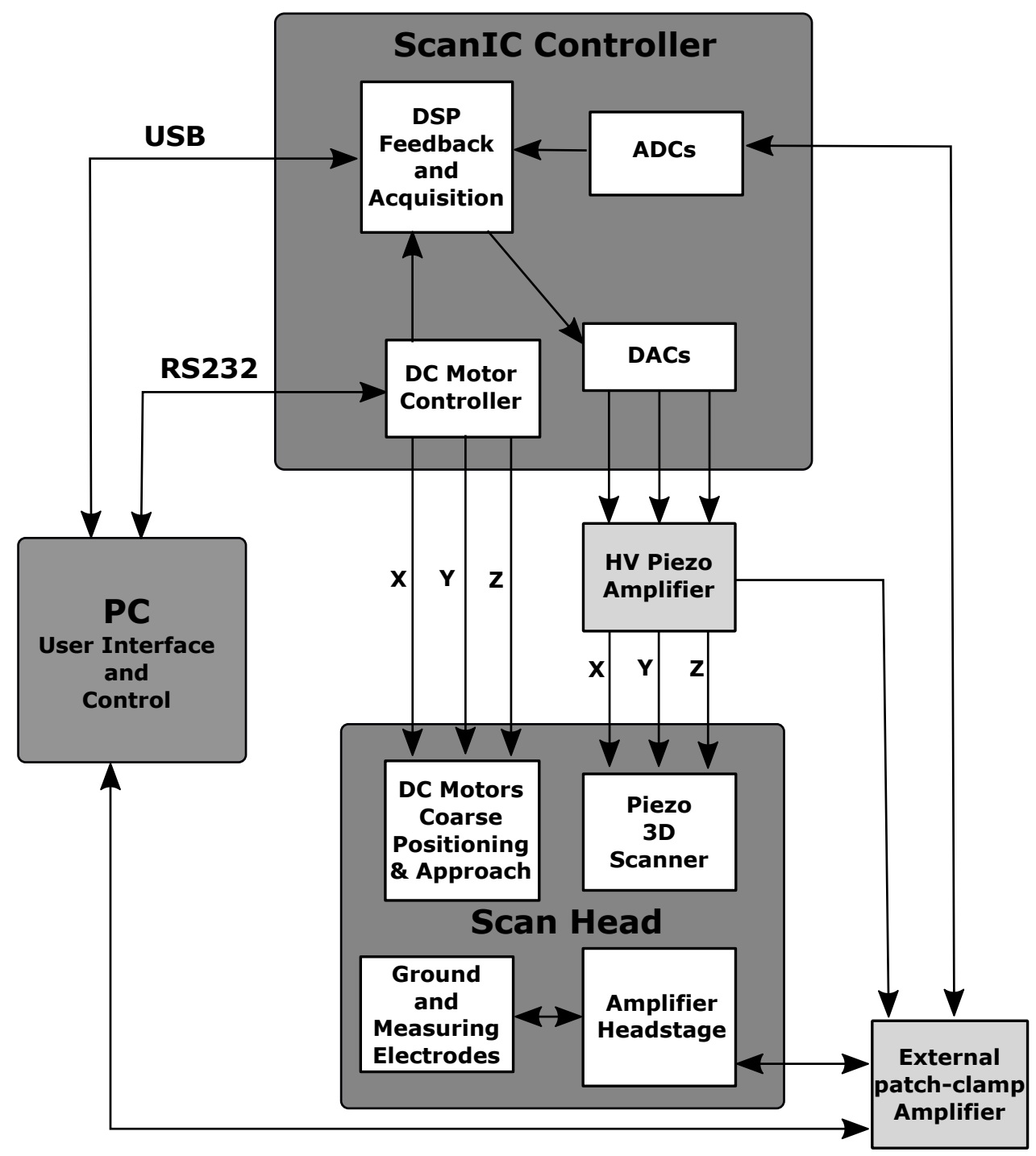

Figure 6.4: Connectivity of the main SICM components. The motors of the scan head are controlled via the SICM controller, while the headstage is controlled and read out by the external amplifier. Both the patch clamp amplifier and the SICM controller are in turn controlled by a personal computer. Adapted from the Ionscope manual. 


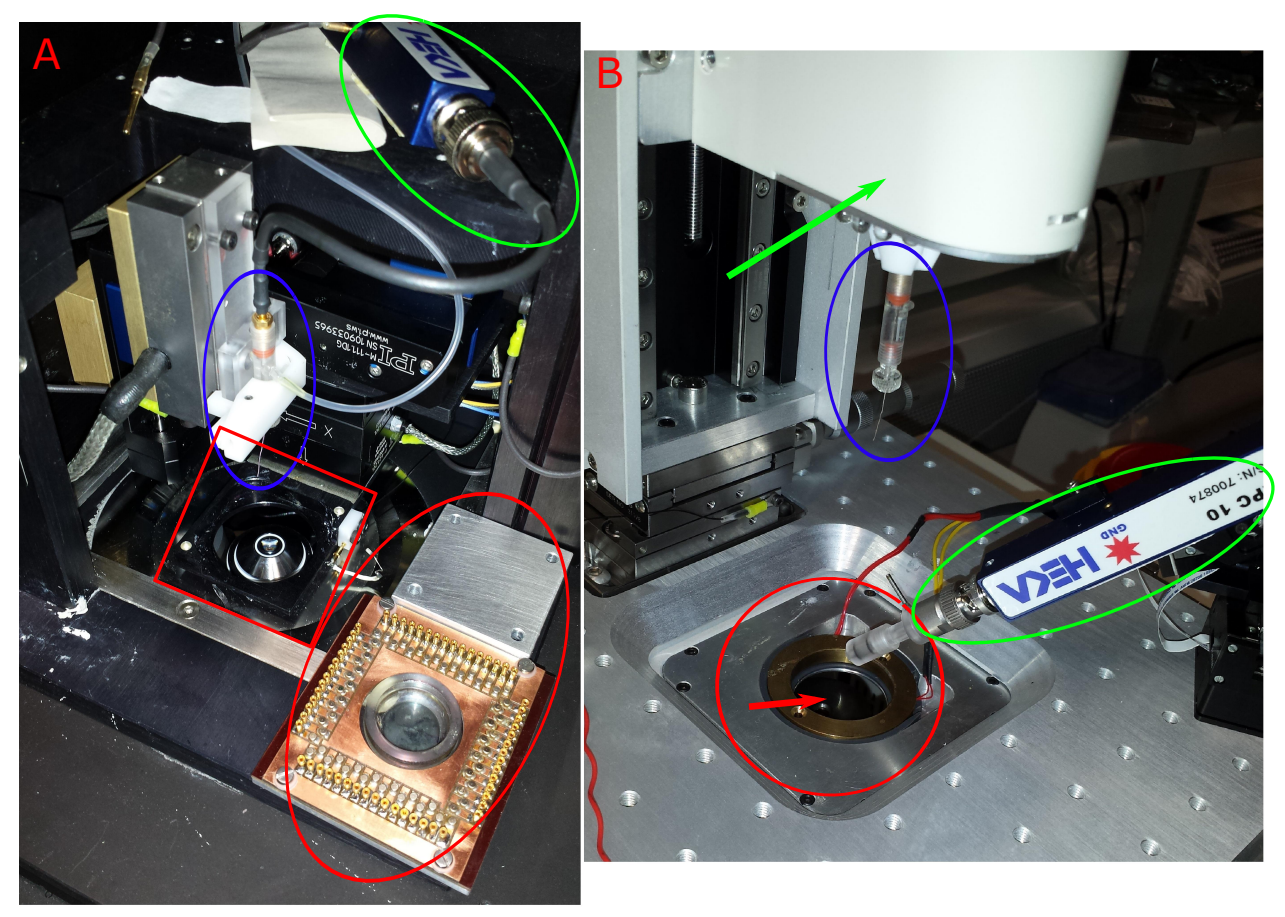

Figure 6.5: SICM scan head. A: Original SICM scan head with opened Faraday cage. The headstage of the amplifier (indicated by the green circle) is connected to the pipette holder (blue circle) and the ground electrode. The pipette is moved in the vertical direction by a coarse motor for immersion and by a piezoelectric stage for vertical movement during scanning. The sample holder (red square) is moved laterally under the pipette during scanning. The custom holder to integrate measurements from MEAs (red circle) is put next to the setup for comparison and can be exchanged for the original holder. B: Scan head of SICM prototype. The recording headstage and the piezos for vertical movement and tilting of the pipette are encased in the shielding indicated by the green arrow. The isolated metal heating ring (red circle) is fitted in a custom holder. A second electrode can be fitted to the headstage (indicated by the green circle), which is held by a micro manipulator. Samples can be illuminated by a mercury lamp or by an high power LED (red arrow). 
currents as in single channel measurements.

This is ideal for combining the SICM technique with single channel recordings, as the currents during SICM imaging are approximately $2 \mathrm{nA}$, while during single channel recordings currents of only several pA are common.

For recording electric potentials the headstage also provides a voltage follower circuit as shown in Figure 2.4 (A). As the amplifier is not optimized for such recordings, the signal-to-noise ratio in the voltage follower mode is not optimal. One of the reasons for this is the lack of a freely tunable band pass filter in our EPC10 as well as the low amplification of the voltage signal. As sensitive potential measurements are needed for recording from the MEAs and even more so for the extracellular recordings we acquired an ELC-03XS (npi electronic GmbH, Tamm, Germany) as additional amplifier with a headstage optimized for voltage clamp at higher currents as encountered in SICM scanning and low signal differential voltage recordings. The ELC-03XS possesses highly tunable low- and high-pass filters for voltage measurements in additions to internal amplification of up to a factor of one thousand. A direct comparison between the recordings using the ECP10 and the ELC-03XS for MEA recordings is given in Figure 6.6.

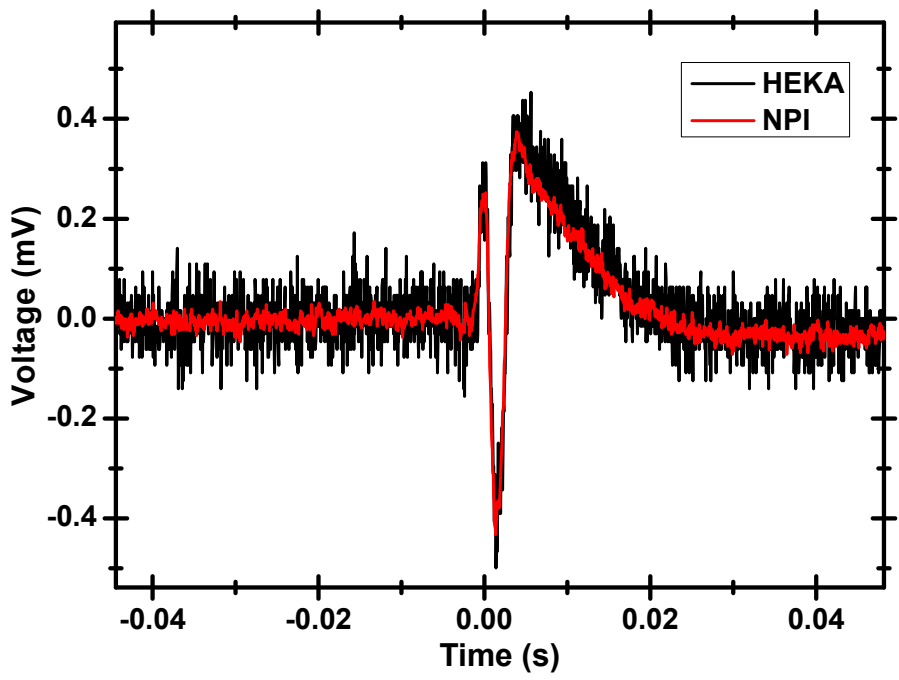

Figure 6.6: Comparison of voltage recording performance. The recorded signal was produced by a MEA test circuit imitating stimulation given by living cells. The low-pass filter of the NPI amplifier was set to the same value as the EPC10 $(10 \mathrm{kHz})$.

\section{Illumination}

Initially, illumination in the SICM system was provided by a standard mercury lamp for fluorescent imaging (Intensilight, Nikon). This was satisfactory for single image fluorescence microscopy, but did not fulfill all the requirements for our experiments. Automated long-time imaging proved impossible as the light source did not possess a shutter which could be driven externally. Using the lamp as light stimulation for the channelrhodopsin experiments was also not feasible, as the light intensity could only be estimated roughly and the light could not be shuttered fast enough for precise illumination control. This is why laser illumination was added to the system. Several iterations of improvements were performed, the final version is given in Figure 6.7. During the ChR experiments illumination at $488 \mathrm{~nm}$ was provided either by a frequency doubled solid state laser (Sapphire, Coherent, Santa Clara, CA) shuttered 


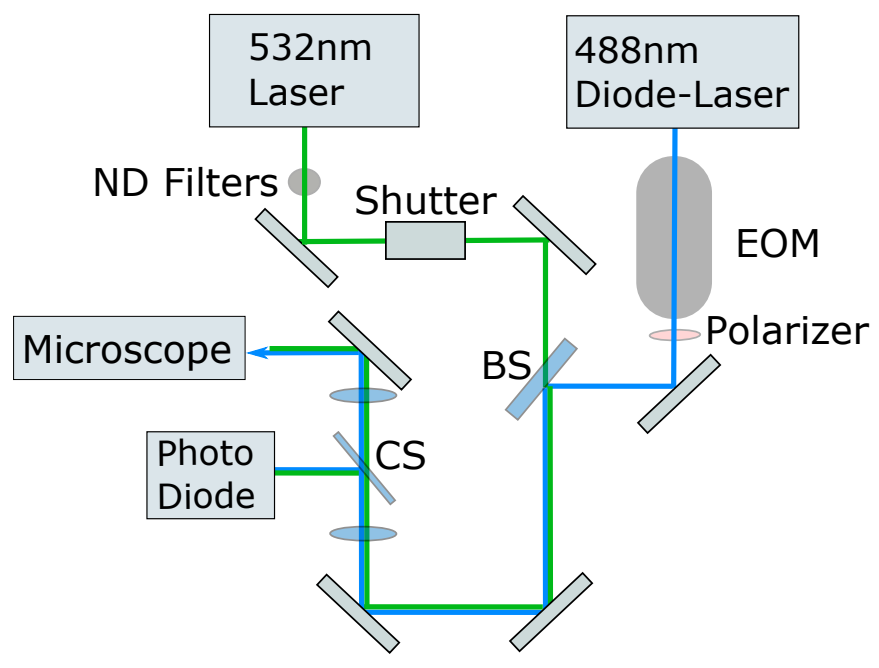

Figure 6.7: Laser illumination light path. Final configuration of the illumination used for the ChR investigation. Green light was provided by a frequency-doubled solid-state laser shuttered by a fast shutter built from the voice coil of a computer hard drive. The intensity was controlled by neutral density filters. Blue light was provided by a diode laser with fast on/off times, so that no additional shutter was required. The intensity can be adjusted by an electro-optic modulator. A photo diode is recording the light waveform from a beam split off by a glass coverslip.

by a custom made, mechanical shutter based on the voice-coil actuator of a hard drive similar to the one described in [105, 106] or by a diode laser (Cobolt MLD, Cobolt AB, Stockholm, Sweden) providing even faster on/off times and making it possible to quickly change amplitudes. Green illumination $(532 \mathrm{~nm})$ was provided by a 'COMPASS 215M-50' (Coherent). The beams of the lasers were coupled into the optical path of the microscope and focused on the sample to a spot size smaller than the field of view of the camera (Coolsnap EZ, Photometrics, Tucson, AZ), to precisely determine the absolute intensity in the plane of the cells as depicted in Figure 6.8.

The laser power was adjusted by neutral density filters, and the absolute power of the laser was measured at the position of the sample with a photo-diode power sensor (PM100D, Thorlabs, Newton, NJ). A photo diode recorded the temporal shape of the laser intensity during the experiments and digitization was performed by the patch-clamp amplifier also used for triggering the laser shutters (EPC10, HEKA, Lambrecht, Germany). The average intensity on each cell was calculated by comparing the size and exact position of the cell with the intensity profile of the laser spot. When using the mechanical shutter, artifacts lasting only two data points were common during switching due to the strong transient currents in the voice coil. These were removed in post processing.

In the latest configuration of the illumination the intensity was adjusted by an electro-optic modulator (EM200, Leysop Ltd., Basildon, UK) driven by a fast highvoltage amplifier (HVA200, Thorlabs), as the diode laser exhibited strong thermal fluctuations when switching to different intensities. 

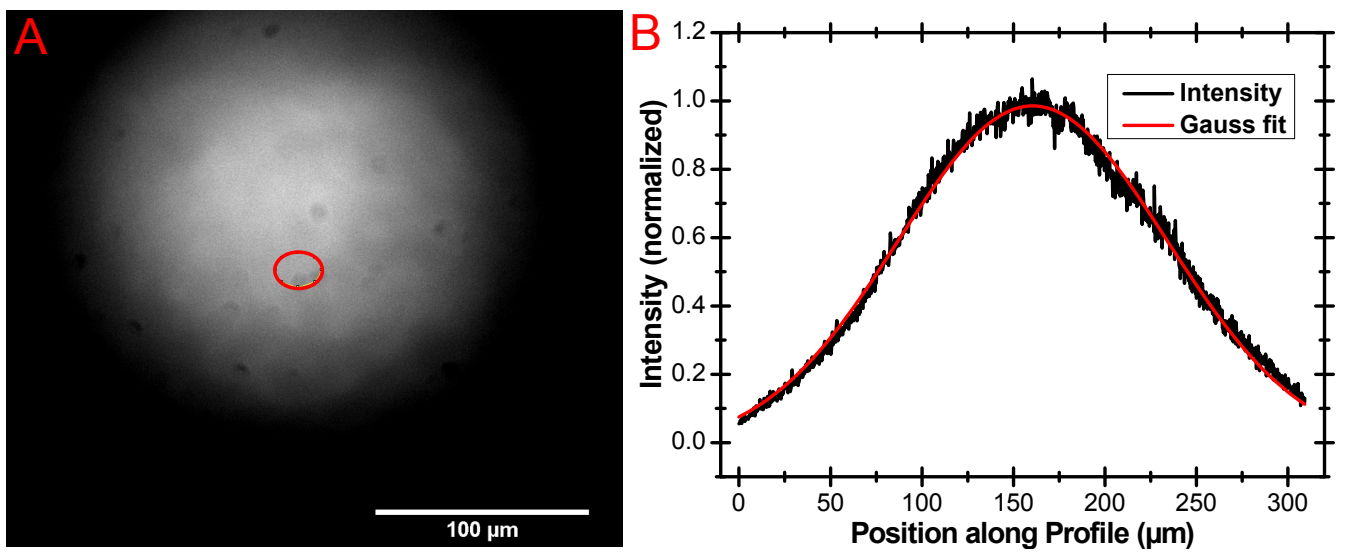

Figure 6.8: Laser illumination at the sample. A: The laser beam is focused to a spot smaller than the field of view. By recording the $2 \mathrm{D}$ intensity profile from the image of a coverslip covered in fluorescent dye, the mean intensity in a small area can be calculated. The red circle indicates the outline of a cell. B: A $1 \mathrm{D}$ profile through the laser spot showing the Gaussian shape.

In the ICnano2043 setup, illumination for fluorescent imaging is provided by an 'EL6000' mercury lamp (Leica). This lamp possesses an internal shutter, which is driven via an 'Arduino Uno' microcontroller (SMART PROJECTS S.r.l., Scarmagno, Italy) controlled by a PC through 'Micro-manager' [107]. Stimulation requiring fast switching of light intensity is provided by a high intensity LED (LXHL-PE02, Luxeon Star LEDs, Brantford, Canada) designed to be fitted into the objective revolver of most microscopes. The design was inspired by a similar light source used by Kai Bröking (MPI DS, Göttingen) and the result is shown in Figure 6.9. Two adjustments were made to suit our needs: Firstly, the LED housing, also acting as cooling element, was fitted with a standard 'TOS link' connector so that an optical cable could be connected to the housing to record the light waveform with high reproducibility. Secondly, my design consists of two distinct parts, so that the actual LED and cables are disconnected from the part being screwed into the microscope. This made it easier to insert the LED and reduced the hassle with twisting cables as the housing is screwed into the microscope.

\subsubsection{Measurement protocols}

\section{Patch clamp measurements}

Coverslips bearing the cells transfected with ChR were fitted into a glass bottom petri dish (Ibidi, Munich, Germany) and submerged in the bath solution. Transfected cells were identified via fluorescence under the inverted microscope (EclipseTi, Nikon, Tokyo, Japan) and the size and position of the cell were recorded via a Coolsnap EZ camera (Photometrics, Tucson, AZ).

Patch-clamp recordings of ChRs were all done in the whole-cell configuration using voltage clamp. If not stated otherwise, the holding potential was $-60 \mathrm{mV}$, and the pipette solution was $110 \mathrm{mM} \mathrm{NaCl}, 10 \mathrm{mM} \mathrm{Na} a_{2} E D T A, 4 \mathrm{mM} M_{g C l}, 10 \mathrm{mM}$ 


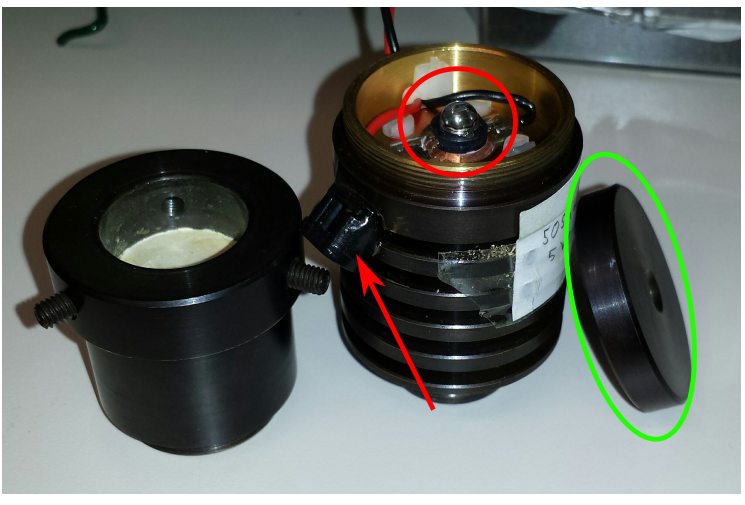

Figure 6.9: LED light source. A highpower LED (red circle) was attached to the top part of the holder with good thermal contact, so that the holder could act as a heat sink. The top cover (green circle) insures that light emission is restricted. Its lower surface is reflective, so that a fraction of the blocked light is redirected into the 'TOS link' connector (red arrow), from which the reference can be collected via a wave guide. On the left is the lower part of the holder which is screwed into the objective revolver of the microscope.

HEPES, $20 \mathrm{mM}$ Glucose, pH 7.4 while the bath solution was $145 \mathrm{mM} \mathrm{NaCl}, 3 \mathrm{mM}$ $\mathrm{KCl}, 1 \mathrm{mM} \mathrm{MgCl}_{2}, 2 \mathrm{mM} \mathrm{CaCl}, 10 \mathrm{mM}$ HEPES, $15 \mathrm{mM}$ Glucose, pH 7.4. If not stated otherwise, the stimulation protocols were created directly in the Patchmaster environment (HEKA) controlling the amplifier. Data was acquired by a HEKA EPC10. Currents were typically low-pass filtered at $4.5 \mathrm{kHz}$ (4-pole Bessel filter) and sampled at $20 \mathrm{kHz}$. For some of the experiments using very short pulses higher frequencies were used, but no difference in the waveform could be observed. Series resistance errors were partially corrected electronically using the amplifiers built-in electronics. Traces were additionally corrected off-line using code similar to that given in [108]. (See section 6.2.4 for a more detailed description.)

\section{Protocol of SICM}

For imaging with SICM the sample has to be fitted into a petri dish and submerged in ionic solution. The dish is placed in the SICM holder and a pipette with sufficiently small opening to achieve the required topographical resolution (see section 3.1) is manufactured and filled with ionic solution. Air pockets can then be removed by applying negative pressure inside the pipette. Depending on the size and shape of the pipette, the process of filling the pipette tip without any remaining air pockets can take several minutes. The filled pipette is then fitted into the pipette holder so that the $\mathrm{Ag} / \mathrm{AgCl}$ electrode reaches the taper. The holder is then inserted into the SICM and pressure is applied to the pipette in order to limit the risk of contamination of the tip during immersion. The amplifier then has to be set to voltage clamp (usually at $200 \mathrm{mV}$ ). The SICM pipette is then lowered into the bath solution until a current between the pipette and the reference electrode is detected. At this point the feedback procedure will start automatically (unless specifically turned off in the software). This means that the piezo motor will start to move up and down by a predefined distance, unless the current is reduced beyond a threshold value (called setpoint in the software and usually between 0.1 and $1 \%$ of the current at the zenith of the hop). Is the threshold reached the pipette will retract at this point, but will 
continue the up and down movement with a smaller amplitude. After the pipette is immersed it is moved into the field of view of the optical microscope. A low magnification needs to be used so that focusing far enough above the cover slip is possible. In the original setup, positioning the pipette is performed by moving the microscope stage, in the prototype the pipette can be moved via a $2 \mathrm{D}$ manual translation stage (Newport). The position of the pipette is then marked on the screen. Thanks to an improved software for the original setup kindly provided to us by Yuri Korchev (Imperial College, London, UK), the position of the pipette is not only marked, but the area of scanning is also shown in the optical image. This option can be calibrated for every camera and magnification. To this end the user has to find a prominent spot on the sample and give the position to the program by clicking on it. The program then moves the piezo stage by a certain amount and the user has to click on the object once more to indicate to the program how many pixel on screen equal the moved distance. For transparent samples, brightfield microscopy and fluorescence microscopy can be used to identify the structure of interest. The sample can be moved without changing the relative position of the pipette to the camera via servo motors controlled by the SICM software. Once the desired structure is found, the pipette is lowered to the sample while the feedback (called hopping mode in software) is active. The feedback will retract the pipette via the vertical piezo stage if the setpoint current is reached, so that the tip will not come in contact with the sample. Once the feedback has detected that the threshold of current reduction has been reached, the downward movement of the pipette's translation stage is automatically stopped. The piezo stage however will continue to move the pipette up and down. At this point the topographic image acquisition can be initialized via the software. As both, the laser illumination and the Leica mercury lamp, can be shuttered by Micro-manager, fluorescent image time-series can be recorded in parallel to the SICM imaging.

\subsubsection{Cell biology}

\section{Cell culture}

Living cells were used for the measurements of the ion currents through photosensitive channels. To this end a line of human embryonic kidney 293 cells (HEK) was cultivated at our lab in an incubator (HERA Cell 150,Thermo Fisher Scientific, Waltham, MA) at $37^{\circ} \mathrm{C}$ and $\mathrm{CO}_{2}$ levels of $5 \%$. The cells grew in $75 \mathrm{~cm}^{2}$ culturing flasks (Sarstedt, Nümbrecht, Germany ) in a medium comprising of DMEM/F12 + GlutaMax (Thermo Fisher Scientific, Waltham, MA) supplemented with $10 \%$ fetal calf serum (Sigma-Aldrich, St. Louis, MO) as nourishment and $1 \%$ penicillin/streptomycin solution to minimize the chance of infection. To prevent the cells from growing into fully confluent layers, they were migrated into different culture flasks every three days. To this end, the cells were submerged in $0.05 \%$ trypsin solution (Sigma-Aldrich, St. Louis, MO) for no more than three minutes. As trypsin digests the adhesion molecules formed by the cells, weak physical vibrations lead to a detachment of the cells from the surface and each other. The free floating cells were then counted under a microscope and the desired number diluted in new medium 
and reseeded in a new culturing flask.

For the investigation of the midbody, MDCK cells stably transfected with GFP tagged human $\alpha$-tubulin were kindly provided by Natalie Elia (Ben-Gurion University, Beer-Sheva, Israel). The cells were cultured in the same manner as HEK cells. The only exception is the culturing medium which consisted of MEM with Earle's salts (F0325, Biochrom, Berlin, Germany ) supplemented with 1\% L-Glutamin, $10 \%$ fetal calf serum and $1 \%$ penicillin/streptomycin solution.

Primary hippocampal neurons were extracted from rat embryos. This mostly took place at the MPI ExpMed, but the appropriate equipment was installed in our institute during the course of this thesis. The cells were cultured on coated glass coverslips in petri dishes at $37^{\circ} \mathrm{C}$ and $\mathrm{CO}_{2}$ levels of $7 \%$ in a separate incubator (BBD6220, Thermo Fisher Scientific).

\section{Transfection}

Transfection of the HEK cells took place during the reseeding process. The required number of cells was collected after trypsinization and transfected with plasmids encoding a ChR2-YFP or a Chronos-GFP fusion protein using a Lonza 4DNucleofector system with the SF cell line Nucleofector kit (Lonza Group Ltd, Basel, Switzerland). The transfection system is based on electroporation, meaning that strong electric fields are increasing the permeability to the cell membrane to the DNA vectors. After transfection the cells were plated on glass cover slips and incubated under the same conditions as the reseeded cells for at least 24 hours.

The pcDNA 3.1-ChR2-YFP construct was kindly provided by Ernst Bamberg (MPI for Biophysics, Frankfurt, Germany), while the Chronos-GFP construct was kindly provided by Edward Boyden (MIT, Cambridge, MA).

\section{AIS staining}

Neurobasal medium (Thermo Fisher Scientific) was used as medium for the procedure. To increase viability of the cells the medium was always supplemented with $10 \mathrm{mM}$ APV [109], a mixture which will be called Neurobasal/APV in the following. As a first step the coverslips with neurons were incubated for five minutes in solution made from a 1:1 mixture of old Neurobasal medium (from the petri dish where the coverslips were taken) and fresh Neurobasal/APV. The cells were then washed with fresh Neurobasal/APV three times before adding the primary antibody in a 1:200 mixture with Neurobasal/APV. After incubating the cells with the primary antibody for five minutes they were washed with fresh Neurobasal/APV three times. Then the secondary antibody was added in a mixture of 1:300 Neurobasal/APV. After 30 seconds the secondary antibodies were washed away with fresh Neurobasal/APV using three washing cycles. 


\subsection{Programing}

A number of programs and automated routines have been created in the course of this thesis in order to evaluate the acquired data. This chapter tries to serves two purposes. Firstly, it provides a general insight into the principles behind the programs and the problems they are created to solve. Secondly, the software itself is described in enough detail to enable a reader to make use of said software.

\subsubsection{Visualization of SICM images}

\section{SICM scanning mode}

The fastest mode of scanning a surface using SICM is to always keep the distance of the probe and sample constant while lateral movement is performed. This is done by adjusting the height of the probe continuously so that the same reduction of the current (by the increase of access resistance) is present. This results in very high lateral resolution in short amounts of time, as the sampling frequency can be high. This fast scanning mode faces two major problems however. As was shown in Figure 6.3, the SICM probe 'senses' a surface at a distance close to the radius of the pipette opening. This means that the distance at which the probe has to be kept would be close to this value. For high resolution imaging this would result in distances of less than $100 \mathrm{~nm}$. This is of little concern for structures possessing only very gradual changes in the topography. If the probe encounters a sudden height increase of more than the radius of the opening however, a lateral collision of pipette and sample is possible. The height of living eukaryotic cells for example can be many micrometers often with a sudden increase of several micrometers at the edges. Collisions between probe and cell are thus nearly unavoidable in the constant distance mode, which leads to false topographies, clogged pipettes and sometimes injured specimen [110. Another problem of the constant distance mode is the gradual change of pipette resistance over time. This is an effect often observed and is usually due to local concentration changes, global concentration changes (due to evaporation of the medium) or small changes of the pipette resistance due to tiny particles attaching to or detaching from it. This means the reference current i.e. the current far away from the surface, should be updated during scanning to ensure that the reduced current reflects a correct increase of the access resistance and thus the sample height.

To circumvent these problems our systems do not use the constant distance mode and instead rely on the 'hopping mode' as illustrated in Figure 6.10. During this mode the pipette moves up and down continuously, updating the reference current every time it reaches its hopping zenith and stopping its approach when the desired reduction relative to this value is reached. During scanning the pipette is not moved continuously over the sample, but instead remains fixed at one sample point during each approach and then moves to the next point during retraction. While the retracting of the pipette during hopping can be very fast, the approach speed needs to be sufficiently slow for the system to detect the sample height before vertical probe sample collision takes place. The hopping mode can thus be considerably 


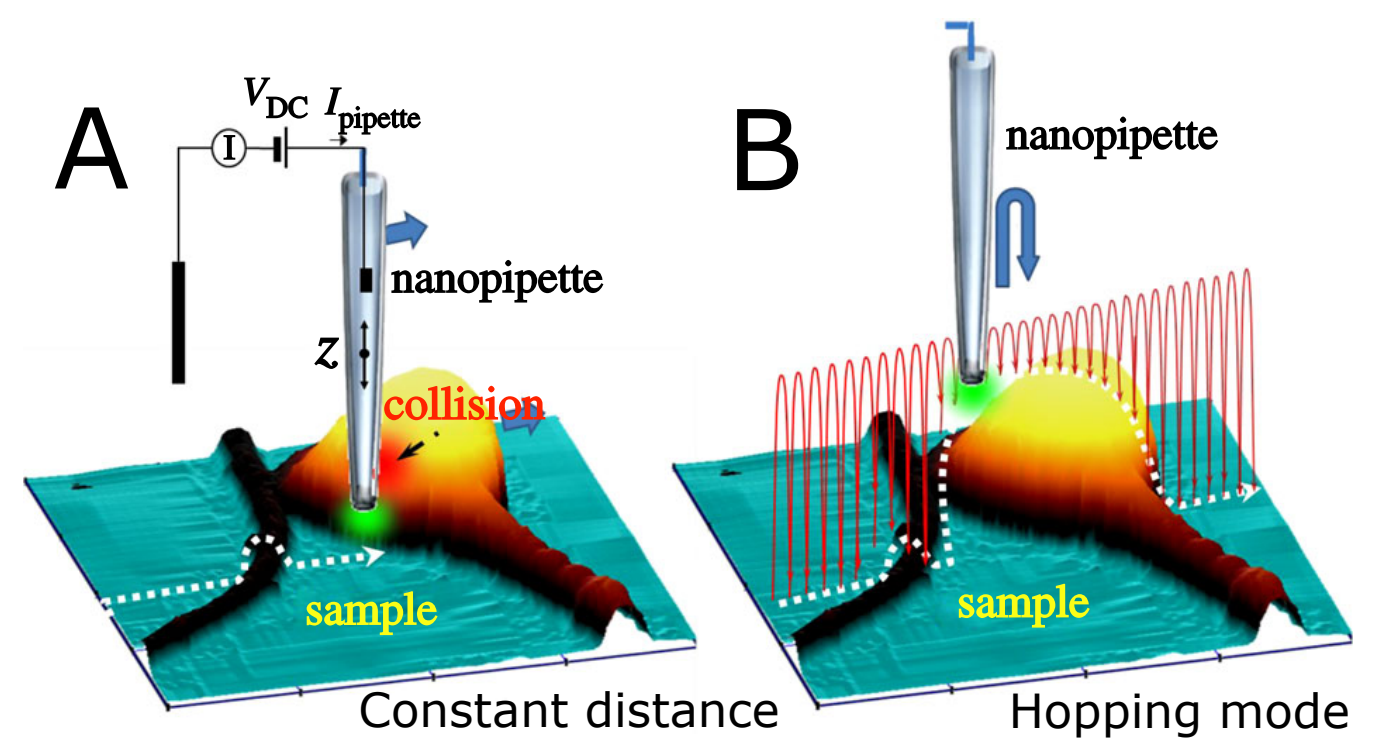

Figure 6.10: Hopping Mode. A: Pipette scanning the sample at a fixed distance. This mode is fast, but sudden height changes larger than the radius of the tip opening often lead to collisions with the sample. B: In the hopping mode the pipette approaches the sample from above and collisions are reduced. By updating the reference current during retraction the system is also less vulnerable to slow changes in pipette conductivity. Taken from [11].

slower than the constant-distance mode, and the required imaging time scales with the hopping height.

It is obvious that the height of hopping has to be larger than the expected change in topography from one sample point to the next. However, the magnitude of the height change is not known until the measurement is actually performed. Both, the original system and the prototype feature an adaptive hopping height nevertheless. The prototype does this by scanning the first line in $\mathrm{x}$ direction using a very high hopping amplitude to avoid collision. During the remaining lines the amplitude is reduced wherever no large height differences were detected in the former scanned lines. This process reduces recording times, but cannot deal with sudden large topography changes in the y direction, since these cannot be deduced from the previous data.

The original system has a more advanced adaptive scanning. Here the image is subdivided into equally sized squares. Initially only the corners of these squares are scanned with a large hopping amplitude. Depending on the heights measured at these corners, the inside of each square is scanned with a lower hop height. This insures that the pipette does not encounter any large topography change with a small hopping amplitude unless the structure is smaller than a single square. To increase the speed of image acquisition a further adaptive strategy is used in the SH01: If the difference between the heights of the square's corners is very small, the number of scans inside that square is reduced. This strategy follows the reasoning, that flat surfaces do not hold any information and thus do not need to be scanned with high resolution. Usually the height difference chosen as threshold for changing between 
high and low resolution is in the range of the noise of the system to identify empty flat surfaces. Figure 6.11 shows the adaptive hopping strategy and an example for the uneven resolution of an image recorded using the adaptive resolution.
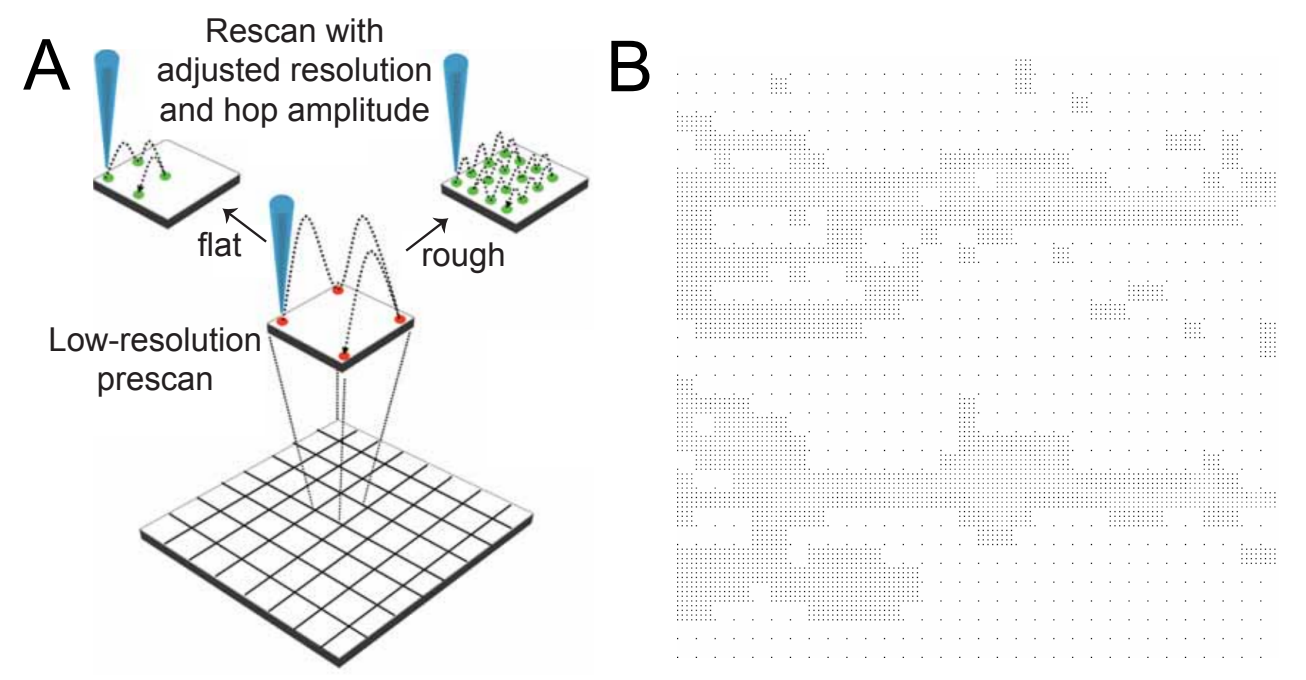

Figure 6.11: Adaptive scanning in the ICnano SH01. A: Adaptive height and resolution in the SH01. The corners of large squares are measured first with high hopping amplitudes. The hop height of the remaining points is adjusted to the height of the sample at the corners. The resolution of the scanning depends on the height difference of the corners. Adapted from [110] B: Raw data from a SICM recording employing adaptive resolution. This image shows a peculiarity of the SH01: It will always create square images although rectangles with full numbered ratios of the side lengths can be chosen. If a non-square rectangle is chosen, the system will record multiple images of that structure until they add up to a full square.

\section{Problems when visualizing data with adaptive resolution}

Due to the irregular spacing of the data points when using adaptive resolution, the data from the SICM scans cannot be evaluated by most software available for topographic images. For this reason the SICM software produces interpolated images along with the raw data. This interpolation fails in some situations however, causing artifacts. This is especially common if an image has been aborted or if a non-square image is recorded as in Figure 6.11 (B). For this reason I programmed a MATLAB (The MathWorks, Natick, MA) function to interpolate the raw data. Figure 6.12 shows a comparison between images from the manufactures software and my own. The first two images show the same image of a neuron soma and its surroundings. The first image (A) is created with the manufacturer's software and the second (B) with the custom MATLAB code. As can be seen, both programs can visualize the data adequately in this case. The vertical lines due to slow drift during scanning are reduce better in the MATLAB program than in the original software however. Figure 6.12 (C) and (D) show the same recording of a curved axon among dendrites. This is an example of an aborted recording which the original software fails to 

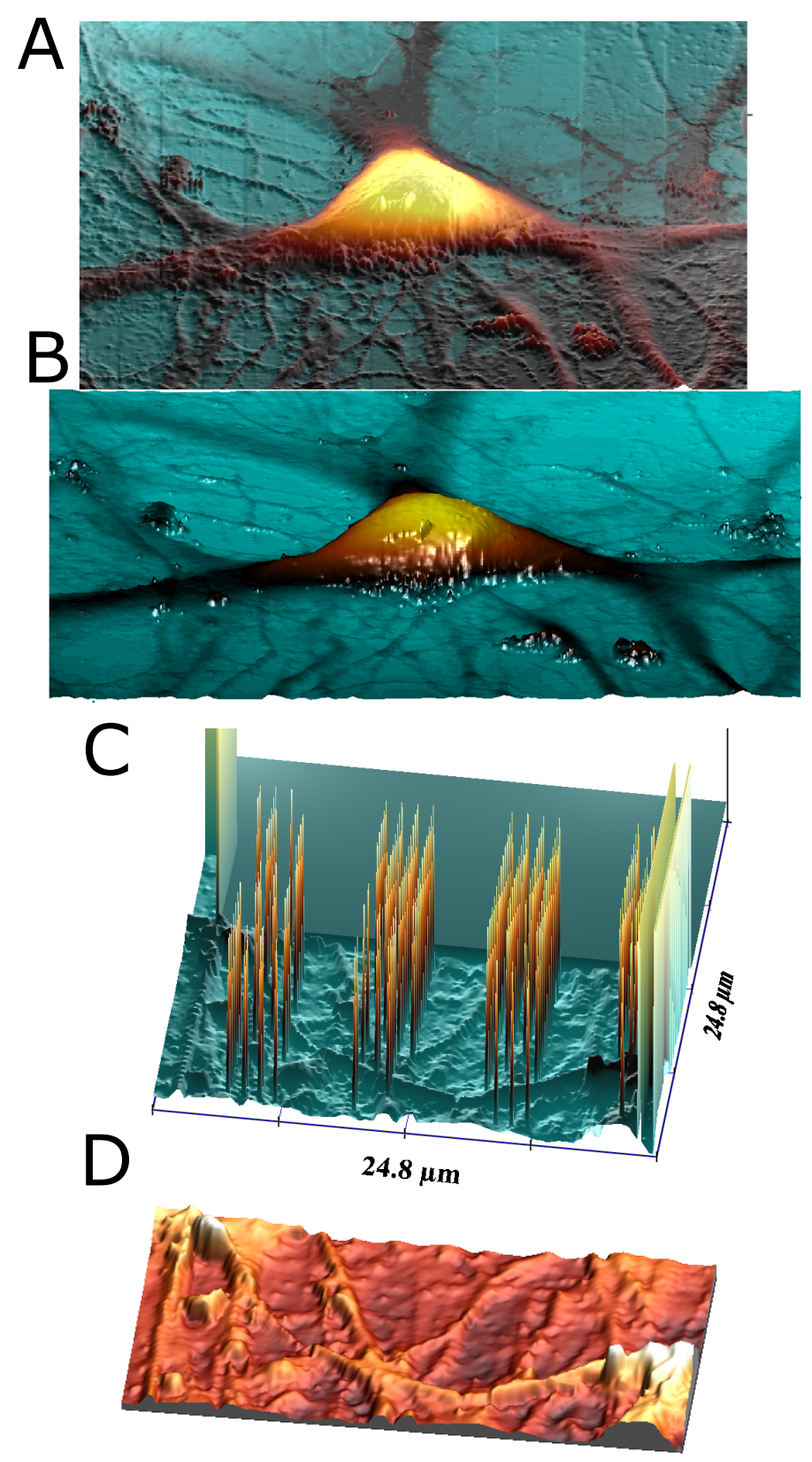

Figure 6.12: Comparison of original software and custom visualization. A: $3 \mathrm{D}$ reconstruction of a neuron soma and its surroundings by the Ionscope software. Vertical stripes are visible due to slow drift. B: The same data as A but visualized with the custom software. The image quality is comparable, but the stripes are removed. C: An example of a recording in which the Ionscope software fails to visualize without artifacts. D: The same data as $\mathbf{C}$ interpolated with the custom software. In this case the image was visualized using 'WsxM' which can import the interpolated data easily. 
interpolate properly $(\mathrm{C})$. In this case the data interpolated with the custom software was visualized with the AFM software 'WsxM' (D). All the artifacts are removed in the custom interpolation.

\section{Custom visualization software}

The code for visualizing the raw SICM data not only interpolates the unevenly distributed data points into an even grid, but also performs several image corrections. The exact principle of the software is as follows. After calling the MATLAB function 'SICM_data_visualizing', a pop-up window will appear asking for the path and filename of the binary file which is to be visualized. After a valid file has been selected the data and meta data will be read from the file. The raw data always consists of $512 \times 512$ data points. The points not measured due to reduced resolution all have the value ' 32767 '. Another window will pop up showing a two dimensional representation of the data as shown in Figure 6.13. The user can decide only to use part of the image, which is necessary in case the image was aborted as in the shown case.

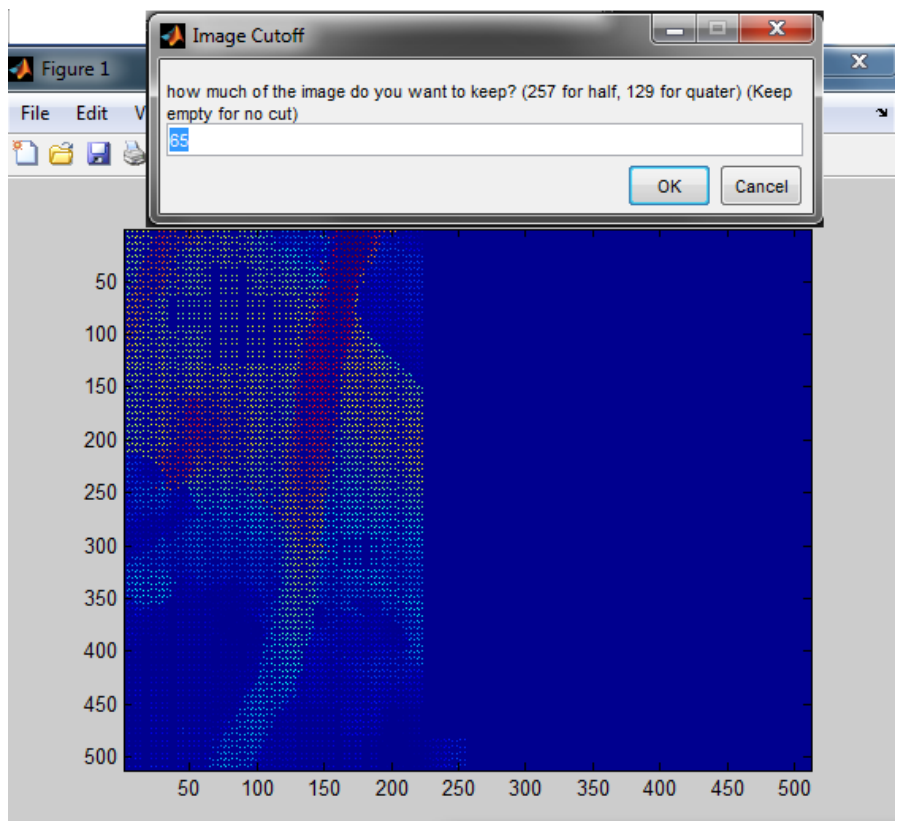

Figure 6.13: Selecting the data to be interpolated. The $2 \mathrm{D}$ representation of the measured points shows the image was aborted, and gives an idea of the imaged structure. The part of the image to the right of the point chosen by the user will be removed from the rest of the image.

After the data has been reduced to the desired size, the program will remove all lines that do not possess any measured point. The remaining points having the value 32767 are then exchanged by NaNs. The image is now usually of a smaller dimension than the raw data and might even be distorted to some degree. This is because the adaptive resolution of the image can lead to parts of the image having more 'empty' lines than others. As all the empty lines have been removed the image in those regions will have a wrong aspect ratio. Image corrections are best performed at this stage of image processing however, as all data points are actual measured heights. The first correction of the data is the removal of the tilt. Tilt is present in nearly all larger images, as the coverslip usually is not perfectly horizontal to the image plane of the pipette. The program incorporates an automatic and a 
manual tilt correction. The automatic tilt correction averages all lines in $\mathrm{x}$ and $\mathrm{y}$ dimension and performs a linear fit to the two resulting curves. Then it corrects for the slope of the linear fits. This procedure only works reliably if the imaged structure is symmetric of course. For this reason it should only be used to save time on larger datasets. The manual version presents an image of the topography to the user. Using the computer mouse a rectangle in a flat region can then be selected in the image. The tilt correction will then only take this flat area into account with very good results. The next step of image correction is the removal of outliers. While not very common, sudden instances of large noise can occur. Such events lead to a false detection of the surface for single points in the image. These can be identified by comparing the height of each point to the height of the surrounding points. Extreme outliers can then be removed. Following the outlier removal the program tries to destripe the image. Stripes due to thermal or other drift occur very often in topographic images by AFM or SICM especially during slow scans. For this reason most AFM software provides destriping algorithms to remove these artifacts. These algorithms are optimized for images created by single line scans however. Since the SH01 scans in lines of larger squares instead of single lines, striping mostly occurs at the edges of these squares as seen in Figure 6.12 (A). Standard procedures for destriping either fail to remove these stripes or distort the image in doing so. The custom MATLAB code deals with this problem specifically by calculating the difference between the two lines forming the edge of two stripes and fitting the result. The fit is then used to correct the offset of the two stripes. The result is shown in Figure 6.12 (B).

After image correction the missing data points are interpolated using the routine 'inpaint_nans' by John D'Erricd ${ }^{1}$. This creates an image with evenly spaced data points. However, the image might still be distorted due to the removal of all empty lines. To correct this, lines are added so that the original aspect ratio is attained. Here it should be recalled that the removed lines are assumed to hold no information as they deviated little from their surroundings. This means that they can be reconstructed from the mean of their surroundings.

The function is called with the command:

$[$ Image, ImagesizeNum $]=S I C M \_d a t a \_v i s u a l i z i n g()$;

Inside the function, flags can be set by changing the following variables either to 1 or 0 : 'spotclean', 'untilt', 'Video' and 'manualdetilt'. The flag 'spotclean' will determine if the function removes outliers, 'untilt' determines if the tilt is removed in the automated fashion and 'manualdetilt' in the manual fashion. The flag 'Video' determines if the second half of an image that is cut in the dialog shown in Figure 6.13 is discarded or also interpolated and saved.

When run, the function creates the matrix 'Image' holding all the data points and a variable 'ImagesizeNum' which hold the information about $\mathrm{x}$ and $\mathrm{y}$ dimensions. These variables, as well as a text file with meta data, are saved to the current MATLAB folder together with an ASCII file that can be imported into more advanced topography image evaluation-software such as WsxM [112] or Gwyddion [113]. To create a three dimensional image from the data in MATLAB, the function:

\footnotetext{
${ }^{1}$ http://de.mathworks.com/matlabcentral/profile/authors/869215-john-d-errico
} 
View_SICM_Image(Image,ImagesizeNum)

can be called.

\subsubsection{Markov models}

Gating of ion channels typically involves the re-arrangements of hundreds of aminoacids. This means it has a vast underlying state-space. To explain the influence of these re-arrangements on a certain observable such as the conductance change not all states need to be considered separately however. This is because many states traversed during the rearrangement can be subsumed into a single transition. This way the original state-space can be reduced to a much smaller space, such as a Markov model with a number of states ranging from 2 to a few dozen. Markov models fulfill the 'Markov property' stating that future changes of the system only rely on the current state of the system, not on the sequence of events that preceded it. In other words, all information about the system is stored within the state of the system itself. The model described in Figure 5.2 for example does not fulfill this property, as the rate of absorption has a time dependency not inherent in the system. Many of the transitions in the Markov models underlying the properties of ion channels are hidden. This is to say the conductance does not change as those transitions take place, but nevertheless they influence the time course of conductance changes at a later time. Often it takes special stimulation protocols to reveal those hidden transitions. The first article in the biophysical journal was devoted to such an endeavor, describing pre-pulse dependence of squid axon's potassium conductance, which is today interpreted as the presence of more than one closed state per potassium channel subunit [114. In such early works the investigators usually had to rely on stimulation protocols that evoked simple responses so that basic fitting routines could be applied. With modern computers whole Markov models can be computed and compared to experimental data, making the approach far more powerful.

\section{Markov model simulation}

The MATLAB functions performing the simulation using Markov models requires the input of an array holding the necessary parameters, an array filled with the time points and a matrix holding the light stimulation which needs to be of the same length as the time vector.

$[$ Currents1,Currents2 $]=$ Simulation $($ Parameter,Time_Vector, Light_Matrix $)$ The parameters differ from model to model and represent either constant rates or absorption constants. The time vector determines the length and the time resolution of the simulation. The light stimulus used in these simulations was derived directly from the light signal recorded during the experiments.

At the start of each simulation the system is initialized to a certain occupation of the states. Usually this is done by only populating the ground state. The occupancies in the system are represented by an array $P(t)$ with a length equal to the number of states. This array always sums to one and is updated at every time step $t$. The change of the population each turn is governed by the transition matrix $T(t)$ made 
up of the transition probabilities $k_{i}(t)$ which are also updated at every time point. The occupancy is updated in the following way:

$$
P(t)=P(t-1)+P(t-1) \times T(t)
$$

At the end of the simulation, vectors holding the entire time course of the states with non-zero conductance are created.

\subsubsection{Finding optimal parameters}

\section{Genetic algorithms}

Genetic algorithms are heuristic optimization routines inspired by natural evolution [115. Unlike many traditional optimization routines, the genetic algorithm does not converge towards a solution with minimal error by calculating the gradient in the solution space and moving along that gradient. Instead, convergence is reached by random recombination of the parameters of the best solutions at each iteration, reminiscent of the recombination of genes in a biological population. The exact mathematical description of genetic algorithms is complicated and has been predominantly investigated for binary 'genes' [116, 117]. It has been shown however, that problems involving real numbers cannot only be solved using appropriate operators but even converge faster than their binomial counterparts. ([118] p.70) Incorporating a genetic algorithm is relatively simple and in theory only requires two things: A 'gene', i.e. a vector of parameters representing a solution and a 'fitness function' to calculate the 'goodness' of the solution. The first step of a genetic optimization is the initiation of the population. The population is the sum of all solutions (also called individuals) present at a single iteration of the algorithm. If no prior information is present then the initial population will be randomly created, ideally spanning a large portion of the state space. The individuals (parameter sets) are then tested for their ability to solve the given problem by the fitness function. For the next iteration some of the solutions are selected while the others are discarded. Numerous methods exist to perform the selection. The best selection scheme and the corresponding 'selection pressure' depends on the specific problem and usually boils down to a trade off between fast convergence to a final solution and a diversity of the population, which decreases the chance of finding only a local optimum. The selection consists of two individual parts: the scaling and the actual selection. In conjuncture, the two processes determine the relation between the fitness and the selection. An important version of the scaling is the 'rank based' variant. This scaling assigns each individual in the population a rank according to its fitness. This seems counter intuitive, as the information about how much better a certain solution can solve the problem is lost. However, due to the desire to keep diversity high as mentioned above, the rank based scaling often performs better than other variants. In fact, it is the scaling mode that performed best in the Markov model optimization done is this work. Of the great number of possible selection methods only the one deemed best in my simulations will be mentioned here: the stochastic remainder sampling. This selection method consists of a deterministic 
and a random part. First, the best individuals of the current generation are chosen in a purely deterministic fashion, then the remaining number will by selected semi-randomly (semi, as the fitness is taken into account as bias). The fraction of deterministically chosen individuals is determined by the scaled fitness distribution [119. When the ranking method is used as scaling, this leads to the same number of deterministically and randomly chosen individuals each iteration. Following the selection procedure, a new generation of individuals is created through three processes called 'mutation', 'crossover' (also called recombination) and 'elitism'. The last process simply takes the best individuals from the former generation into the next. Mutation causes one or more of the parameters of an individual to change in a random fashion, and crossover creates a new individual from two or more individuals from the last generation. Each of the three processes serves a specific goal. Elitism insures that good solutions are not lost from one iteration to the next. Crossover is a means of convergence, as it probes for better solutions between already good solution, while mutation decreases the chance of only finding a local optimum by increases the portion of the solution space searched. Once enough individuals have been created, the next iteration is performed in the same manner as the first.

\section{Description of optimization software}

To optimize the parameters determining the transition rates in the Markov model described in section 6.2.2, a genetic algorithm based on the publicly available version by De Freitas $2^{2}$ was employed.

The general structure of the algorithm was kept, although some modifications and fixes were added to the original code. The two most noteworthy changes are a change of the initialization and the implementation of an additional crossover routine. The initialization was designed to create individuals with starting parameters not randomly distributed but gathered in a Gauss distribution around given starting values. The width of the gauss distribution depends on the allowed minimal and maximal value of each parameter. The added crossover routine was designed to not just exchange parameters between parents (as is the only option in a binary system) but to create a value in between those of the parents. The whole optimizing routine is called in MATLAB with

$[$ Population, stats, settings, Problem $]=$ Call_GA(Chr2_initialize(Parameters, Limit, Time, Light, Data))

The optimization requires the following inputs: a vector with an initial parameter guess, a matrix holding the upper and lower boundaries for each parameter, a vector giving all time points, a matrix with the light stimulation and a matrix with the recorded data to which the simulations should be compared. Adding another recorded data trace and the corresponding light vector as input behind the last argument adds it to the global optimization. This is the only way to use traces of a different length. The output of the function consists of four structures: the popu-

\footnotetext{
${ }^{2}$ http://sourceforge.net/projects/gatoolbox/?source=navbar
} 
lation of the final iteration, some statistics of the genetic algorithm, the settings of the genetic algorithm and all data and settings of the simulation and optimization. Due to the complexity of the program, arising from the large number of options and parameters, I added a graphical interface giving an overview of some of the most relevant options in the initiation function. A screen shot of the interface is given in Figure 6.14.

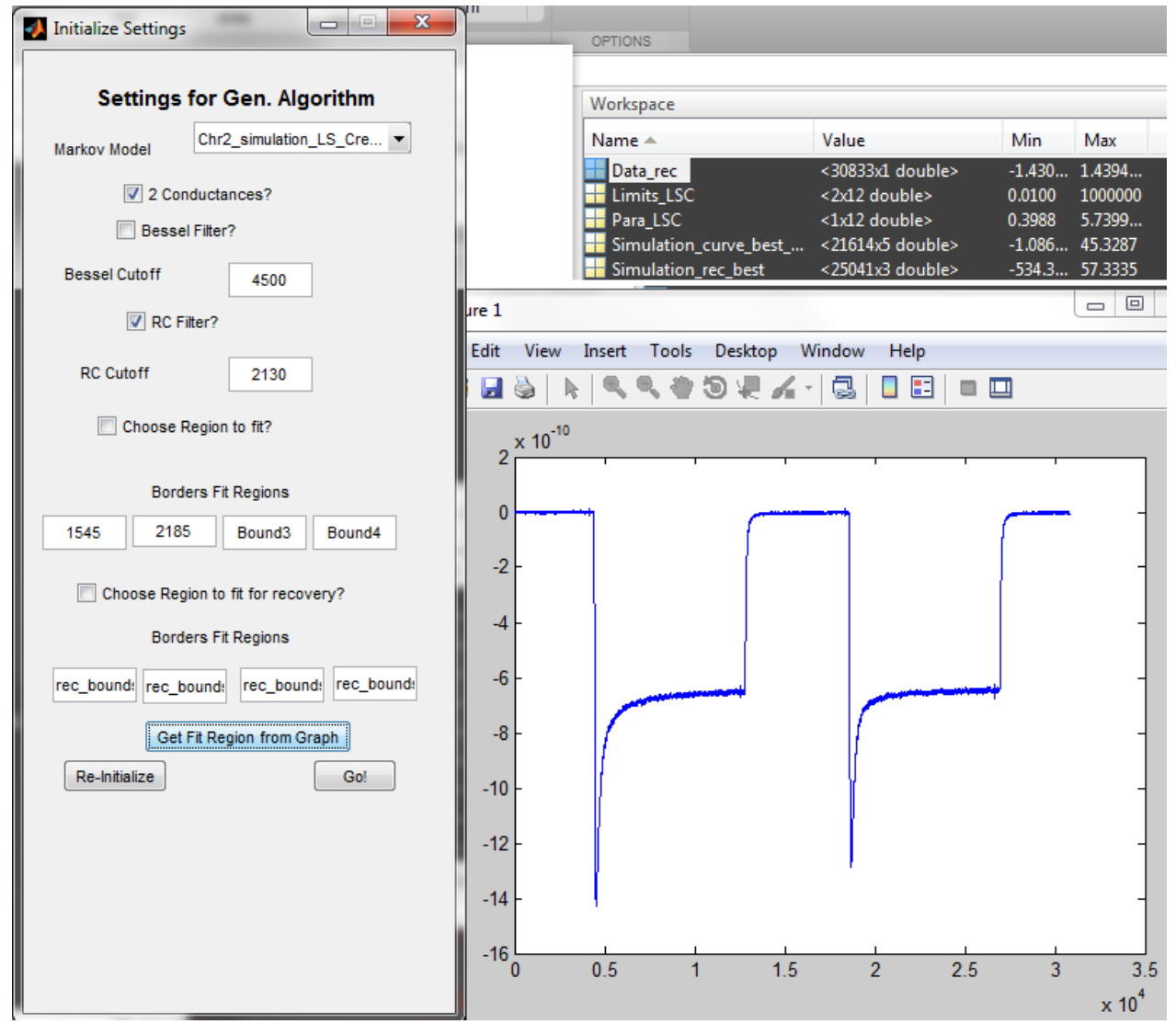

Figure 6.14: GUI of the optimization. The most important parameters that can be entered in the code of the initiation routine are shown and can be altered in the controls shown on the left. Clicking the 'Get Fit Region from Graph' button opens a graph showing the recorded data as seen on the right. If another data set with a different length was added when calling this function, this data is shown after the 'Return' key is pressed, which accepts the chosen points. The procedure of choosing the part of the trace of the second data set is the same as for the first. The fit region for the second data set is displayed in the boxes labeled 'rec_bounds'.

Optimization is performed by minimizing the squared difference between the simulation trace and that of the recorded data. This means, that the scaling factor (representing the single-channel conductivity multiplied by the amount of functional channels) has a dominating effect, making convergence of the genetic optimization slow. For this reason the scaling parameter was dissociated from the genetic optimization and the factor with the minimal simulation-to-measurement difference was calculated and used. For models possessing two conducting states with differ- 
ent conductances, an additional parameter needs to be dissociated from the general optimization. Ticking the ' 2 conductances?' box will perform this dissociation for two separate conductance values. The next two options determine how much the simulated data is filtered before it is compared to the real recorded data. The real data is both actively filtered by a 4 pole Bessel filter, and involuntarily by the series resistance. These filters can also be implemented for the simulated data and the cutoff frequency for both can be specified in the graphical interface. The eight lower boxes can be used to restrict the regions of the traces which are to be compared. In the example shown in Figure 6.14 only the data between the 1545th and the 2185th time point would be compared. If the flag 'Coose Region to fit?' is ticked only the data between the first two and last two bounds is compared. The region to be compared can also be chosen from a graph of the data. To do this the button 'Get Fit Region from Graph' must be pressed. This opens a graph window as seen on the right of Figure 6.14. Clicking in the graph will select a point and pressing the 'return' key will enter these points into the appropriate boxes. After pressing the 'Go!' button the graphical interface of the genetic algorithm will open as shown in Figure 6.15.

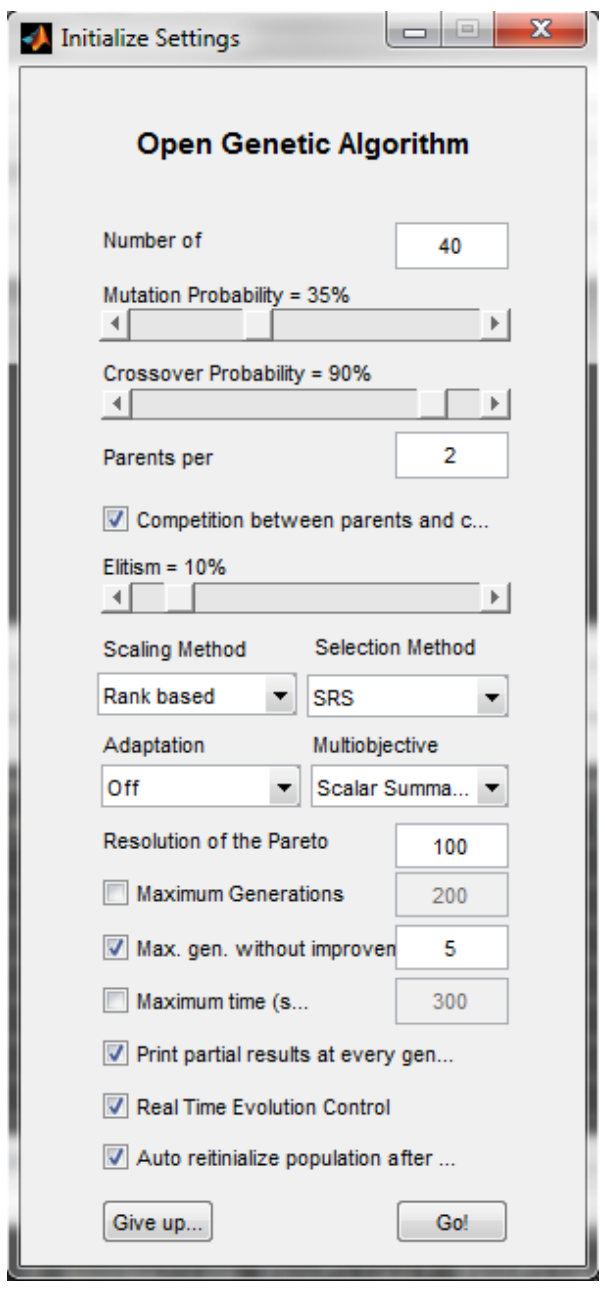

Figure 6.15: Genetic algorithm GUI. Before the optimization commences, all the settings of the genetic algorithm are presented and can be altered from this interface.

This interface is part of the original code by De Freitas and gives the user control over all the parameters discussed in section 6.2.3, such as the number of individuals 
in the population, the mutation and crossover probabilities, the parents per child, ect. After pressing the 'Go!' button on this screen the optimization starts and provides a visual representation of the progress as well as the option to change certain parameters while optimization is in progress, as shown in Figure 6.16.

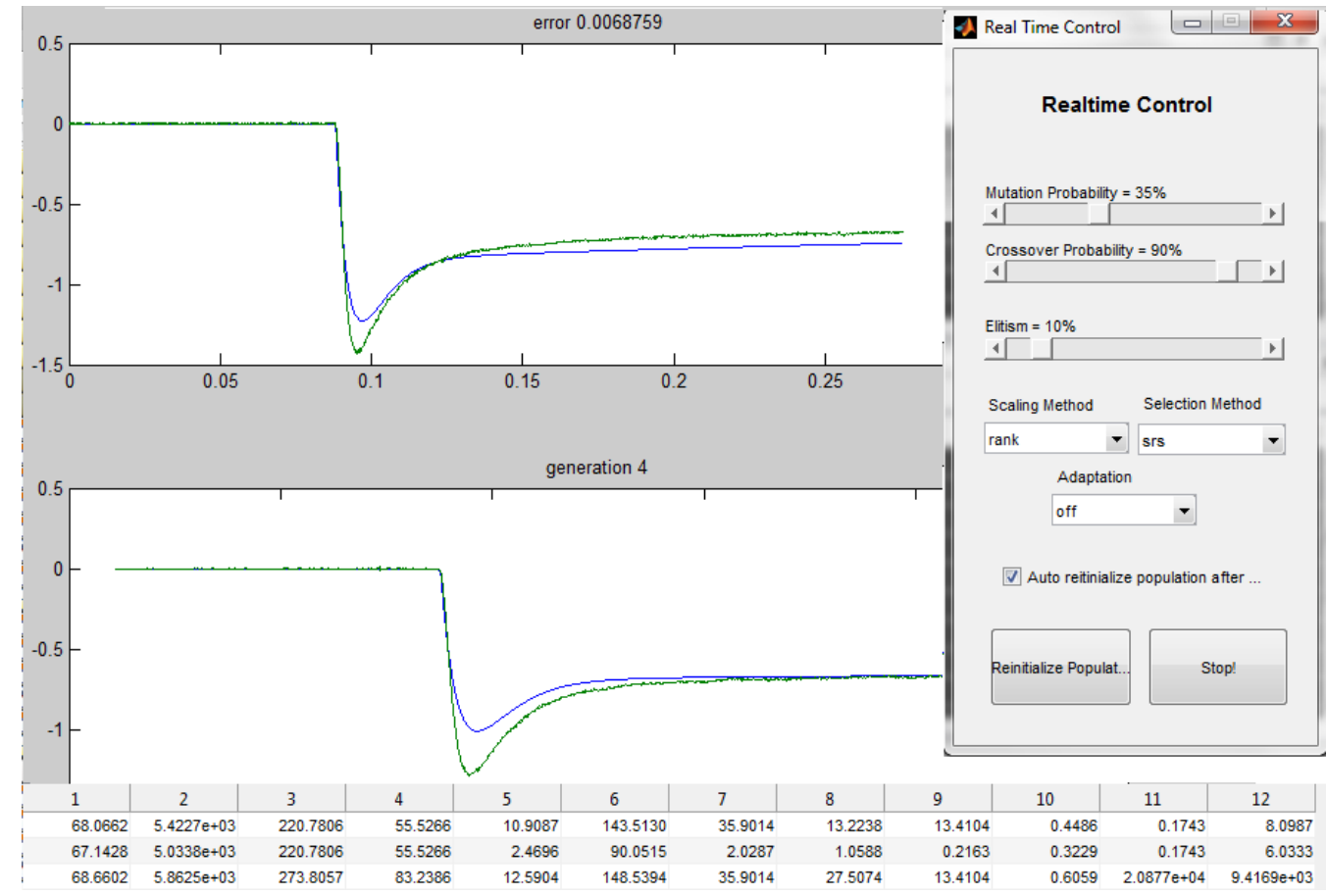

Figure 6.16: GA realtime control. After each iteration the best solution of that iteration will be graphically overlaid with the part of the data (green trace) it is compared to. The graph also displays the error of that solution (top) and the number of the iteration (between the curves). At the bottom the parameter set of the best solution is listed as well as the minimum and maximum value of each parameter present in the current generation. This gives an indication of the diversity of the population. The window on the right allows the user to change some of the GA parameters for future iterations.

\section{Metrics determining goodness of fit}

In this study, optimization of the Markov models took place by minimizing the differences between the waveform of the simulation and the actual currents as opposed to certain parameters of the response as in previous studies [47, 120. The major drawback of fitting parameters instead of whole traces is the inevitable bias towards certain properties of the response. For example, choosing the maximum- and steady state current as parameter but not the transition between those two points would lead to a poor representation of the responses to light pulses of several $100 \mathrm{~ms}$, as the response curve to such stimuli is dominated by the current after the peak but before equilibrium. 
In an earlier attempt to evaluate our data, the ratio between the peaks of two sequential pulses, as shown in Figure 5.6(B), was used as a parameter to determine how well our model reproduced the effect of light adaptation. Later comparison of the actual traces revealed that this lead to an overestimation of how well certain models fitted the data. This was due to the fact that while the peak current was the same for both model and experiment, the rise time in the simulation differed considerably.

This, naturally, also leads to wrong assumptions about the underlying molecular transitions of the molecule. Such issues are reduced by introducing as many relevant parameters as possible to confine the model parameters well. However, even with enough parameters to completely confine the current trace, a certain bias still exists due to the problem of weighting each parameter. Values such as the time constant of the initial current rise have to be globally minimized alongside values of a completely different nature, such as the ratio between peak and steady-state current. This requires a metric to determine what minimizing the global difference between simulation and recorded data actually means.

By minimizing the differences between the entire waveforms the bias introduced during the minimization is reduced. However, bias still exists due to the stimulation protocols used. If a certain feature is predominantly probed for in the data (for example many very long pulses compared to short ones), it will be weighted stronger during optimization. In general however, using the entire trace for optimization tends to cause less bias and, more importantly, has a lower risk of losing information. It is also usable for strongly time-dependent stimuli, for which parameters such as time constants are hard to determine.

\subsubsection{Data analysis}

\section{Correction of series resistance}

The series resistance errors described in section 2.2 can in theory be corrected computationally after recording [108]. In practice this would require knowing the exact reversal potential and I/V relation, since simple assumptions such as linear I/V curves can only be used for small corrections. Using the electronic on-line correction described in section 2.4 is the safer option as it does not require such assumptions. It can not compensate the error fully due to the instability of the positive feedback however.

For the evaluation of the ChR data I created a MATLAB function called ' $C$ onvert_for_Origin' that averages traces, removes jitter caused by the shutter and creates fits for the activation and deactivation time constants if square light pulses were used. This function also incorporates different kinds of series-resistance errorcorrections. Depending on the options chosen it can use code similar to that given in [108] to compensate for the reduced voltage and the low pass filtering. Instead of the latter it can also 'boost' the high frequencies, that were lost due to the low-pass filtering, by inverting the low-pass filter. For the latter the self-written function: $[$ Numerator, Denominator $]=R C \_$compensation $\left(\right.$sample $_{f} r e q$, cutof $f$, Boost $)$ was used. This function either creates a $R C$ low-pass filter or (if the variable 'Boost' 
is given) a high-frequency boost compensating for said filter. Every correction of the high-frequency loss naturally increases the noise considerably. For this reason I decided to use the electronic compensation to reduce the series resistance error by 40 to $70 \%$ and correct for the remaining voltage error using code similar to that given in [108]. The low-pass filtering was not compensated for computationally. Instead the theoretical data (both simulations and fits) were low-pass filtered before being compared to the recorded data. This proved to be far more effective.

\section{Time constant calculations}

When performing fits to traces of electrophysiological data, the filtering of the recorded signal has the same negative influence as in the optimization of the Markov simulations. To counter this effect the same strategy of filtering the fitting curves

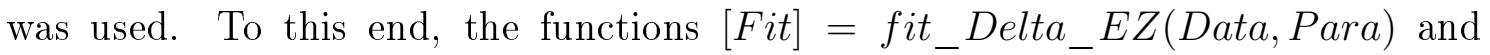
$[F i t]=$ fit_exp_EZ(Time,Data,Num,Den) were created. The first function fits the data of the current response to a short light flash as shown in Figure 5.4. Its input is a matrix holding the time vector, the data vector, the light stimulation (as time stamp) and a vector holding the information for the filter creation. The second function performs an exponential fit filtered by a filter determined by the numerator and denominator given. Fits are performed in MATLAB (The MathWorks, Natick, MA) partially using Frederic Moisys fitting routine 'EZfit ${ }^{3}$. This fitting routine made integration of the filtering function especially easy.

\section{Spike averaging}

Increasing the reliability of data by performing many instances of the measuring procedure is common practice. One of the most basic principles of evaluating multiple measurements is simple averaging. In the case of a trace as was shown in Figure 4.4 (B) for example, averaging is straight forward. All that is required is a time stamp to align the traces and averaging can be performed separately for every position in the trace. This becomes even easier if a single peak is present. In this case the minimum or maximum can be used as time stamp for aligning. However, if noise is present the peak is not necessarily the maximal value. Several possibilities exist to circumvent this problem. I have implemented one method in the function

\section{$[$ ACurve 1, ACurve 2$]=$ Spikeaverage $($ Trace $)$.}

Its input is a vector holding a trace with multiple occurrences of the signal to be averaged. In this function the time stamp for averaging two curves is determined in the following way: Several values in close proximity to the peak are considered as candidates for the time stamp, and the area difference between the two curves is calculated for each. The time stamp with the smallest divergence is then chosen. For each trace added to the average, the second curve used for calculating the area difference is updated to be the average of the traces added up to this point. The traces shown in Figure $4.4(\mathrm{~B}, \mathrm{C})$ were all averaged in this fashion.

\footnotetext{
${ }^{3}$ http://www.fast.u-psud.fr/ moisy/ml/
} 


\section{Additional Software used}

Some additional 'commercial' software was used in the course of this study. For creating graphs and performing simple fits and statistics Origin Pro (OriginLab Corporation, Northampton, MA) was used. For visualizing topographic videos WSxM [112] was used due to its feature of tracking structures in topographic image series. For evaluation of the topographic images created with the prototype system SPIP (Image Metrology A/S, Hørsholm, Denmark) was used. Integration of the live feed from the microscope into the software of the original SICM was achieved via the image grabber VCam (e2eSoft, Shanghai, P.R.China). Images for the thesis were created using Blender ${ }^{4}$ Inkscape ${ }^{5}$ and Gimp ${ }^{6}$. The text was written with Sublime Text 3 (Sublime HQ Pty Ltd, Sydney, Australia).

\footnotetext{
${ }^{4}$ https://www.blender.org

${ }^{5}$ https://inkscape.org

${ }^{6}$ http://www.gimp.org/
} 


\section{Summary and Outlook}

Characterizing the properties of ion channels in biological membranes was the main objective during the course of this thesis. Two systems were investigated: The conductance changes of the membrane of the axon initial segment due to fast sodium channels, and the light sensitive ion channel Chronos.

The latter investigation focused purely on the properties of the light gated channels and not, for example, their distribution in the membrane. As the vector holding the Chronos gene can be inserted into simple, small, round cells such as HEK cells, the electrophysiological measurements could be performed with standard patch-clamp equipment and techniques. The evaluation of the data needed to extract single molecule parameters is highly complex however, and was performed by optimizing newly developed Markov models using an elaborate optimization routine. Using this approach, the currents mediated by Chronos could be reproduced with high precision, and several interesting properties of the molecular transitions were discovered. The most notable being the relation of Chronos' fast kinetics at low intensities to the short lifetimes of its conducting states as compared to ChR2. This leads to a lower opening probability for Chronos than for ChR2. To achieve similar depolarization, more channels or higher single-channel conductance are thus necessary for Chronos. Another interesting effect that was characterized is the relatively weak light adaptation of Chronos. This reduces the light intensities needed for stimulation shortly after prior illumination.

An additional goal would be creating a hybrid of Chronos and ChR2, that combines the fast kinetics of the first with the reliability of the latter. While some structure/function relationships have been found in this study, more detailed information about the influence of specific molecular structures is needed. Some of this information can be gathered by investigating channels created from point mutations of the wild types. This can be partially accomplished using the software developed for this thesis.

For the AIS investigation the opposite problem of the ChRs investigation was faced. A program capable of performing the main part of the computational analysis had already been created by Andres Agudelo-Toro and was available to us. The electrophysiological measurements on the other hand are highly complicated, as they have to be performed in living neurons and not only need to discern single molecule properties but also the distribution of these molecules in the membrane. This requires the determination of the geometrical structure of the cell as well as highly sensitive measurements at numerous positions. A setup capable of performing these tasks has been created and a proof of principle has been conducted for every step, including the topographical imaging at acceptable speed, the high-precision positioning of 
electrodes, the stimulation via Chronos and low-noise potential measurements. So far, the important step of extracting the membrane parameters of the AIS has not been performed. The greatest obstacle is acquiring enough data from a single neuron to describe the extra cellular action potentials with enough precision. Improvements to the SICM software are crucial for this task as the stock software employed in our new system not only slows down the data recording speed, but also leads to more collisions of probe and sample than the older system. With the possibility of modifying the software ourselves, most of these problems can be resolved. Since Korchev's group at the Imperial College in London has already devised several methods of improving the performance, such as faster scanning modes and better optical-to-SICM calibration, improving our own setup is reduced to a pure programming task. 


\section{List of Figures}

2.1 The cell membrane as a circuit. . . . . . . . . . . . . . . . 4

2.2 Configurations of the patch-clamp technique. . . . . . . . . . . . . . 9

2.3 Operational amplifier with negative feedback. . . . . . . . . . . . . 10

2.4 Patch-clamp amplifier circuits. . . . . . . . . . . . . . . 12

3.1 Access resistance at a surface. . . . . . . . . . . . . . . . . . 14

3.2 Bridge connecting two cells before abscission. . . . . . . . . . . . . . 16

3.3 Fluorescent microscopy of midbody. . . . . . . . . . . . . . . . . . . 19

3.4 Midbody profiles. . . . . . . . . . . . . . . . 20

3.5 Line scanning of midbody. . . . . . . . . . . . . . . 21

4.1 Action potential generation. . . . . . . . . . . . 25

$4.2 \quad$ Action potential propagation in the AIS. . . . . . . . . . . . 30

4.3 AIS investigation. . . . . . . . . . . . . . . . . 32

4.4 Averaging of distance dependent waveform. . . . . . . . . . . . 35

$5.1 \quad$ Light cycle of ChR2 as derived from optical spectroscopy. . . . . . . . 39

5.2 Branched model as seen in the literature. . . . . . . . . . . . . . . . 40

5.3 Chronos displays a faster light response than ChR2. . . . . . . . . . . . . . 43

5.4 Impulse Response. . . . . . . . . . . . . . . . . . . . 45

5.5 Simple closed loop model. . . . . . . . . . . . . . . . . . . . 46

5.6 Light adaptation. . . . . . . . . . . . . . . . . . . . . 48

5.7 Markov model of the light cycle of ChR2/Chronos. . . . . . . . . . 50

5.8 Optimized traces of Chronos/ChR2. . . . . . . . . . . . . . 52

5.9 Hybrid Evaluation. . . . . . . . . . . . . . . . . 53

5.10 Voltage dependencies. . . . . . . . . . . . . . . . 56

5.11 Bleach model. . . . . . . . . . . . . . . . . . . 57

5.12 Bleaching. . . . . . . . . . . . . . . . . 58

5.13 Comparison to other models. . . . . . . . . . . . . 60

6.1 Pipette resistance. . . . . . . . . . . . . . . . 63

6.2 EM image of pipette. . . . . . . . . . . . . . . . . . . 64

6.3 Approach curve analysis. . . . . . . . . . . . . . . 66

6.4 Connectivity of the main SICM components. . . . . . . . . . . . . . 69

6.5 SICM scan head. . . . . . . . . . . . . . . . . 70

6.6 Comparison of voltage recording performance. . . . . . . . . . . . 71

6.7 Laser illumination light path. . . . . . . . . . . . . . . . . . . . . . . . . . . . . . 72

6.8 Laser illumination at the sample. . . . . . . . . . . . . 73

6.9 LED light source. . . . . . . . . . . . . . . . . . . . . . 74 
6.10 Hopping Mode. . . . . . . . . . . . . . . . . . . 78

6.11 Adaptive scanning in the ICnano SH01. . . . . . . . . . . . . . . . . . 79

6.12 Comparison of original software and custom visualization. . . . . . 80

6.13 Selecting the data to be interpolated. . . . . . . . . . . . . . . 81

6.14 GUI of the optimization. . . . . . . . . . . . . . . . . . . . . 86

6.15 Genetic algorithm GUI. . . . . . . . . . . . . . . . . 87

6.16 GA realtime control. . . . . . . . . . . . . . . . . . 88 


\section{Abbreviations}

AIS axon initial segment

SICM scanning ion conductance microscopy

MEA multi electrode array

LIF leaky integrate and fire

LED light emitting diode

op-amp operational amplifier

IR infrared

AP action potential

eAP extracellular action potential

iAP intracellular action potentials

ChR channelrhodopsin

MEM minimum essential medium

DMEM Dulbecco's minimum essential medium

HEPES 4-(2-hydroxyethyl)-1-piperazineethanesulfonic acid

APV Amino-phosphonovalerate

EDTA Ethylenediaminetetraacetic acid

AFM atomic force microscopy

MDCK Madin-Darby canine kidney

HEK human embryonic kidney

NaN not a number

GUI graphical user interface

GFP green fluorescent protein

YFP yellow fluorescent protein 
EM electron microscope

SIM structured illumination microscopy

ESCRT endosomal sorting complexes required for transport 


\section{Bibliography}

[1] A. L. Hodgkin and A. F. Huxley, "A quantitative description of membrane current and its application to conduction and excitation in nerve," J. Physiol., vol. 117 , no. 4, pp. 500-544, 1952.

[2] E. Neher and B. Sakmann, "Single-channel currents recorded from membrane of denervated frog muscle fibres," Nature, vol. 260, 1976.

[3] E. Marder, "Understanding brains: Details, intuition, and big data," PLoS Biol., vol. 13, 052015.

[4] S. Kunkel, M. Schmidt, J. M. Eppler, H. E. Plesser, G. Masumoto, J. Igarashi, S. Ishii, T. Fukai, A. Morrison, M. Diesmann, and M. Helias, "Spiking network simulation code for petascale computers," Front Neuroinform, vol. 8, p. 78, 2014 .

[5] M. S. Lewicki, "A review of methods for spike sorting: the detection and classification of neural action potentials," Network, vol. 9, pp. R53-R78, Nov 1998 .

[6] M. A. L. Nicolelis, D. Dimitrov, J. M. Carmena, R. Crist, G. Lehew, J. D. Kralik, and S. P. Wise, "Chronic, multisite, multielectrode recordings in macaque monkeys," PNAS, vol. 100, pp. 11041-11046, Sep 2003.

[7] R. C. Kelly, M. A. Smith, J. M. Samonds, A. Kohn, A. B. Bonds, J. A. Movshon, and T. S. Lee, "Comparison of recordings from microelectrode arrays and single electrodes in the visual cortex," J. Neurosci., vol. 27, pp. 261-264, Jan 2007.

[8] I. H. Stevenson and K. P. Kording, "How advances in neural recording affect data analysis," Nat. Neurosci., vol. 14, pp. 139-142, Feb 2011.

[9] A. Neef, A. E. Hady, J. Nagpal, K. Broking, G. Afshar, O. Schlüter, T. Geisel, E. Bamberg, R. Fleischmann, W. Stühmer, and F. Wolf, "Continuous dynamic photostimulation -inducing in-vivo-like fluctuating conductances with channelrhodopsins," ArXiv.

[10] L. M. Palmer, B. A. Clark, J. Gründemann, A. Roth, G. J. Stuart, and M. Häusser, "Initiation of simple and complex spikes in cerebellar purkinje cells," J. Physiol., vol. 588, no. 10, pp. 1709-1717, 2010. 
[11] A. Agudelo-Toro and A. Neef, "Computationally efficient simulation of electrical activity at cell membranes interacting with self-generated and externally imposed electric fields," J. Neural. Eng., vol. 10, no. 2, p. 026019, 2013.

[12] R. Sherman-Gold, The Axon guide for electrophysiology $\&$ biophysics: laboratory techniques. Axon Instruments, 1993.

[13] P. G. Nelson and M. Lieberman, Excitable cells in tissue culture. Springer Science \& Business Media, 2012.

[14] C. Taylor, "Microelectrodes and micromagnets," Exp. Biol. Med, vol. 23, no. 2, pp. $147-150,1925$.

[15] S. Gelfan, "A non-polarizable micro-electrode. preliminary report.," Exp. Biol. Med, vol. 23, no. 4, pp. 308-309, 1926.

[16] R. A. Jortner, S. S. Farivar, and G. Laurent, "A simple connectivity scheme for sparse coding in an olfactory system," J. Neurosci., vol. 27, no. 7, pp. 16591669, 2007.

[17] B. Sakmann, Single-channel recording. Springer Science \& Business Media, 2013.

[18] M. H. P. Kole, S. U. Ilschner, B. M. Kampa, S. R. Williams, P. C. Ruben, and G. J. Stuart, "Action potential generation requires a high sodium channel density in the axon initial segment," Nat. Neurosci., vol. 11, pp. 178-186, Feb 2008.

[19] B. Barbour, "Electronics for electrophysiologists," 2014.

[20] A. Strickholm, "Impedance of a small electrically isolated area of the muscle cell surface," J. Gen. Physiol., vol. 44, no. 6, pp. 1073-1088, 1961.

[21] O. P. Hamill, A. Marty, E. Neher, B. Sakmann, and F. Sigworth, "Improved patch-clamp techniques for high-resolution current recording from cells and cell-free membrane patches," Pflügers Archiv, vol. 391, no. 2, pp. 85-100, 1981.

[22] P. Hansma, B. Drake, O. Marti, S. Gould, and C. Prater, "The scanning ionconductance microscope," Science, vol. 243, no. 4891, pp. 641-643, 1989.

[23] H. Nitz, J. Kamp, and H. Fuchs, "A combined scanning ion-conductance and shear-force microscope," Probe microsc, vol. 1, pp. 187-200, 1997.

[24] M. A. Edwards, C. G. Williams, A. L. Whitworth, and P. R. Unwin, "Scanning ion conductance microscopy: a model for experimentally realistic conditions and image interpretation," Anal. Chem., vol. 81, pp. 4482-4492, 2009.

[25] D. Pastre, H. Iwamoto, J. Liu, G. Szabo, and Z. Shao, "Characterization of AC mode scanning ion-conductance microscopy," Ultramicroscopy, vol. 90, pp. 13-19, may 2001. 
[26] J. Rheinlaender and T. E. Schäffer, "Image formation, resolution, and height measurement in scanning ion conductance microscopy," J. Appl. Phys., vol. 105, 2009.

[27] R. Howland, L. Benatar, and C. Symanski, A Practical Guide to Scanning Probe Microscopy. DIANE Publishing Company, 1998.

[28] G. Binnig, C. F. Quate, and C. Gerber, "Atomic force microscope," Phys. Rev. Lett., vol. 56, pp. 930-933, Mar 1986.

[29] J. Rheinlaender, N. A. Geisse, R. Proksch, and T. E. Schäffer, "Comparison of scanning ion conductance microscopy with atomic force microscopy for cell imaging," Langmuir, vol. 27, pp. 697-704, Jan 2011.

[30] J. Seifert, J. Rheinlaender, P. Novak, Y. E. Korchev, and T. E. Schäffer, "Comparison of atomic force microscopy and scanning ion conductance microscopy for live cell imaging," Langmuir, vol. 31, pp. 6807-6813, Jun 2015.

[31] N. Elia, R. Sougrat, T. A. Spurlin, J. H. Hurley, and J. Lippincott-Schwartz, "Dynamics of endosomal sorting complex required for transport (ESCRT) machinery during cytokinesis and its role in abscission," PNAS, vol. 108, pp. 48464851, Mar 2011.

[32] P. Markiewicz and M. C. Goh, "Atomic force microscopy probe tip visualization and improvement of images using a simple deconvolution procedure," Langmuir, vol. 10, no. 1, pp. 5-7, 1994.

[33] A. Bukharaev, N. Berdunov, D. Ovchinnikov, and K. Salikhov, "Threedimensional probe and surface reconstruction for atomic force microscopy using a deconvolution algorithm," In Practice, vol. 5, p. 21, 1998.

[34] A. I. Shevchuk, G. I. Frolenkov, D. Sánchez, P. S. James, N. Freedman, M. J. Lab, R. Jones, D. Klenerman, and Y. E. Korchev, "Imaging proteins in membranes of living cells by high-resolution scanning ion conductance microscopy," Angew. Chem. Int. Ed., vol. 45, no. 14, pp. 2212-2216, 2006.

[35] J. Gorelik, Y. Gu, H. A. Spohr, A. I. Shevchuk, M. J. Lab, S. E. Harding, C. R. W. Edwards, M. Whitaker, G. W. J. Moss, D. C. H. Benton, D. Sanchez, A. Darszon, I. Vodyanoy, D. Klenerman, and Y. E. Korchev, "Ion channels in small cells and subcellular structures can be studied with a smart patch-clamp system," Biophys. J., vol. 83, pp. 3296-3303, 122002.

[36] A. K. Dutta, Y. E. Korchev, A. I. Shevchuk, S. Hayashi, Y. Okada, and R. Z. Sabirov, "Spatial distribution of maxi-anion channel on cardiomyocytes detected by smart-patch technique," Biophys. J., vol. 94, pp. 1646-1655, March 2008.

[37] Y. E. Korchev, Y. A. Negulyaev, C. R. Edwards, I. Vodyanoy, and M. J. Lab, "Functional localization of single active ion channels on the surface of a living cell," Nat. Cell Biol., vol. 2, pp. 616-619, September 2000. 
[38] A. Bruckbauer, D. Zhou, L. Ying, Y. E. Korchev, C. Abell, and D. Klenerman, "Multicomponent submicron features of biomolecules created by voltage controlled deposition from a nanopipet," J. Am. Chem. Soc., vol. 125, no. 32, pp. 9834-9839, 2003. PMID: 12904050.

[39] L. Ying, A. Bruckbauer, A. M. Rothery, Y. E. Korchev, and D. Klenerman, "Programmable delivery of DNA through a nanopipet," Anal. Chem., vol. 74, no. 6, pp. 1380-1385, 2002. PMID: 11922307.

[40] A. I. Shevchuk, J. Gorelik, S. E. Harding, M. J. Lab, D. Klenerman, and Y. E. Korchev, "Simultaneous measurement of Ca21 and cellular dynamics: Combined scanning ion conductance and optical microscopy to study contracting cardiac myocytes," Biophys. J., vol. 81, pp. 1759-1764, 2001.

[41] Y. E. Korchev, M. Raval, M. J. Lab, J. Gorelik, C. R. Edwards, T. Rayment, and D. Klenerman, "Hybrid scanning ion conductance and scanning near-field optical microscopy for the study of living cells," Biophys J., vol. 78, no. 5, pp. 2675-2679, 2000.

[42] T. E. Schaeffer, "Nanomechanics of molecules and living cells with scanning ion conductance microscopy," Anal. Chem., 2013.

[43] J. Rheinlaender and T. E. Schäffer, "Mapping the mechanical stiffness of live cells with the scanning ion conductance microscope," Soft Matter, vol. 9, no. 12, pp. 3230-3236, 2013.

[44] H. Neto and G. W. Gould, "The regulation of abscission by multi-protein complexes," J. Cell Sci., vol. 124, pp. 3199-3207, Oct 2011.

[45] N. Elad, S. Abramovitch, H. Sabanay, and O. Medalia, "Microtubule organization in the final stages of cytokinesis as revealed by cryo-electron tomography," J. Cell Sci., vol. 124, pp. 207-215, Jan 2011.

[46] J. W. Connell, C. Lindon, J. P. Luzio, and E. Reid, "Spastin couples microtubule severing to membrane traffic in completion of cytokinesis and secretion," Traffic, vol. 10, pp. 42-56, Jan 2009.

[47] J. C. Williams, J. Xu, Z. Lu, A. Klimas, X. Chen, C. M. Ambrosi, I. S. Cohen, and E. Entcheva, "Computational optogenetics: Empirically-derived voltageand light-sensitive channelrhodopsin-2 model," PLoS Comput. Biol., vol. 9, 2013.

[48] M. R. Zimmermann, H. Maischak, A. Mithöfer, W. Boland, and H. H. Felle, "System potentials, a novel electrical long-distance apoplastic signal in plants, induced by wounding," Plant Physiol., vol. 149, pp. 1593-1600, Mar 2009.

[49] J. Fromm and S. Lautner, "Electrical signals and their physiological significance in plants," Plant Cell Environ., vol. 30, pp. 249-257, Mar 2007. 
[50] A. H. Delcour, "Electrophysiology of bacteria," Annu. Rev. Microbiol., vol. 67, pp. 179-197, 2013.

[51] J. B. Miller and D. Koshland, Jr, "Sensory electrophysiology of bacteria: relationship of the membrane potential to motility and chemotaxis in bacillus subtilis," PNAS, vol. 74, pp. 4752-4756, Nov 1977.

[52] B. A. Clark, P. Monsivais, T. Branco, M. London, and M. Häusser, "The site of action potential initiation in cerebellar purkinje neurons," Nat. Neurosci., vol. 8, no. 2, pp. 137-139, 2005.

[53] C. M. Colbert and E. Pan, "Ion channel properties underlying axonal action potential initiation in pyramidal neurons," Nat. Neurosci, vol. 5, no. 6, pp. 533-538, 2002.

[54] F. A. Azevedo, L. R. Carvalho, L. T. Grinberg, J. M. Farfel, R. E. Ferretti, R. E. Leite, R. Lent, S. Herculano-Houzel, et al., "Equal numbers of neuronal and nonneuronal cells make the human brain an isometrically scaled-up primate brain," J. Comp. Neurol., vol. 513, no. 5, pp. 532-541, 2009.

[55] J. DeFelipe, P. Marco, I. Busturia, and A. Merchán-Pérez, "Estimation of the number of synapses in the cerebral cortex: Methodological considerations," Cerebral Cortex, vol. 9, no. 7, pp. 722-732, 1999.

[56] E. D. Adrian, "The impulses produced by sensory nerve-endings," J. Physiol., vol. 62, no. 1, pp. 33-51, 1926.

[57] B. W. Knight, "Dynamics of encoding in a population of neurons," J. Gen. Physiol., vol. 59, no. 6, pp. 734-766, 1972.

[58] M. Carandini, D. J. Heeger, and J. A. Movshon, "Linearity and normalization in simple cells of the macaque primary visual cortex," J. Neurosci., vol. 17, no. 21 , pp. $8621-8644,1997$.

[59] C. E. Stafstrom, P. C. Schwindt, and W. E. Crill, "Repetitive firing in layer V neurons from cat neocortex in vitro," J. Neurophysiol., vol. 52, no. 2, pp. 264$277,1984$.

[60] N. J. Priebe and D. Ferster, "Mechanisms of neuronal computation in mammalian visual cortex," Neuron, vol. 75, no. 2, pp. 194-208, 2012.

[61] N. Fourcaud-Trocmé, D. Hansel, C. Van Vreeswijk, and N. Brunel, "How spike generation mechanisms determine the neuronal response to fluctuating inputs," J. Neurosci., vol. 23, no. 37, pp. 11628-11640, 2003.

[62] N. Fourcaud-Trocmé and N. Brunel, "Dynamics of the instantaneous firing rate in response to changes in input statistics," J. Comput. Neurosci., vol. 18, no. 3, pp. 311-321, 2005. 
[63] B. Naundorf, T. Geisel, and F. Wolf, "Action potential onset dynamics and the response speed of neuronal populations," J. Comput. Neurosci., vol. 18, no. 3, pp. 297-309, 2005.

[64] T. Tchumatchenko, A. Malyshev, F. Wolf, and M. Volgushev, "Ultrafast population encoding by cortical neurons," J. Neurosci., vol. 31, pp. 12171-12179, August 2011.

[65] G. Testa-Silva, M. B. Verhoog, D. Linaro, C. P. J. de Kock, J. C. Baayen, R. M. Meredith, C. I. De Zeeuw, M. Giugliano, and H. D. Mansvelder, "High bandwidth synaptic communication and frequency tracking in human neocortex," PLoS Biol., vol. 12, p. e1002007, Nov 2014.

[66] W. Wei and F. Wolf, "Spike onset dynamics and response speed in neuronal populations," Phys. Rev. Lett., vol. 106, no. 8, p. 088102, 2011.

[67] G. Baranauskas, Y. David, and I. A. Fleidervish, "Spatial mismatch between the $\mathrm{Na}+$ flux and spike initiation in axon initial segment," Proc. Natl. Acad. Sci. U.S.A., vol. 110, pp. 4051-4056, March 2013.

[68] C. Gold, D. A. Henze, and C. Koch, "Using extracellular action potential recordings to constrain compartmental models," J. Comput. Neurosci., vol. 23, no. 1, pp. 39-58, 2007.

[69] C. Gold, D. A. Henze, C. Koch, and G. Buzsáki, "On the origin of the extracellular action potential waveform: a modeling study," J. Neurophysiol., vol. 95, no. 5, pp. 3113-3128, 2006.

[70] J. Noguchi, A. Nagaoka, S. Watanabe, G. C. R. Ellis-Davies, K. Kitamura, M. Kano, M. Matsuzaki, and H. Kasai, "In vivo two-photon uncaging of glutamate revealing the structure-function relationships of dendritic spines in the neocortex of adult mice," J Physiol, vol. 589, pp. 2447-2457, May 2011.

[71] S. Shoham, D. H. O'Connor, D. V. Sarkisov, and S. S.-H. Wang, "Rapid neurotransmitter uncaging in spatially defined patterns," Nat. Methods, vol. 2, pp. 837-843, Nov 2005.

[72] E. M. Callaway and R. Yuste, "Stimulating neurons with light," Curr. Opin. Neurobiol., vol. 12, pp. 587-592, Oct 2002.

[73] R. L. Fork, "Laser stimulation of nerve cells in aplysia," Science, vol. 171, pp. 907-908, Mar 1971.

[74] E. M. Callaway and L. C. Katz, "Photostimulation using caged glutamate reveals functional circuitry in living brain slices," $P N A S$, vol. 90, pp. 76617665, Aug 1993.

[75] M. Banghart, K. Borges, E. Isacoff, D. Trauner, and R. H. Kramer, "Lightactivated ion channels for remote control of neuronal firing," Nat. Neurosci., vol. 7, pp. 1381-1386, Dec 2004. 
[76] F. Zhang, L.-P. Wang, E. S. Boyden, and K. Deisseroth, "Channelrhodopsin-2 and optical control of excitable cells," Nat. Methods, vol. 3, p. 785, 2006.

[77] G. Nagel, D. Ollig, M. Fuhrmann, S. Kateriya, A. M. Musti, E. Bamberg, and P. Hegemann, "Channelrhodopsin-1: a light-gated proton channel in green algae," Science, vol. 296, pp. 2395-2398, Jun 2002.

[78] G. Nagel, T. Szellas, W. Huhn, S. Kateriya, N. Adeishvili, P. Berthold, D. Ollig, P. Hegemann, and E. Bamberg, "Channelrhodopsin-2, a directly lightgated cation-selective membrane channel," PNAS, vol. 100, no. 24, pp. 1394013945, 2003.

[79] S. Q. Lima and G. Miesenböck, "Remote control of behavior through genetically targeted photostimulation of neurons," Cell, vol. 121, pp. 141-152, Apr 2005 .

[80] L. Madisen, T. Mao, H. Koch, J.-m. Zhuo, A. Berenyi, S. Fujisawa, Y.-W. A. Hsu, A. J. Garcia, 3rd, X. Gu, S. Zanella, J. Kidney, H. Gu, Y. Mao, B. M. Hooks, E. S. Boyden, G. Buzsáki, J. M. Ramirez, A. R. Jones, K. Svoboda, X. Han, E. E. Turner, and H. Zeng, "A toolbox of cre-dependent optogenetic transgenic mice for light-induced activation and silencing," Nat. Neurosci., vol. 15, pp. 793-802, May 2012.

[81] L. A. Gunaydin, O. Yizhar, A. Berndt, V. S. Sohal, K. Deisseroth, and P. Hegemann, "Ultrafast optogenetic control," Nat. Neurosci., 2010.

[82] A. Dawydow, R. Gueta, D. Ljaschenko, S. Ullrich, M. Hermann, N. Ehmann, S. Gao, A. Fiala, T. Langenhan, G. Nagel, and R. J. Kittel, "Channelrhodopsin-2-xxl, a powerful optogenetic tool for low-light applications," PNAS, vol. 111, pp. 13972-13977, Sep 2014.

[83] A. Berndt, O. Yizhar, L. A. Gunaydin, P. Hegemann, and K. Deisseroth, "Bi-stable neural state switches," Nat. Neurosci., vol. 12, pp. 229-234, 2009.

[84] N. C. Klapoetke, Y. Murata, S. S. Kim, S. R. Pulver, A. Birdsey-Benson, Y. K. Cho, T. K. Morimoto, A. S. Chuong, E. J. Carpenter, Z. Tian, et al., "Independent optical excitation of distinct neural populations," Nat. Methods, vol. 11, no. 3, pp. 338-346, 2014.

[85] C. Bamann, T. Kirsch, G. Nagel, and E. Bamberg, "Spectral characteristics of the photocycle of channelrhodopsin-2 and its implication for channel function," J. Mol. Biol., vol. 375, no. 3, pp. 686-694, 2008.

[86] E. Ritter, K. Stehfest, A. Berndt, P. Hegemann, and F. J. Bartl, "Monitoring light-induced structural changes of channelrhodopsin-2 by UV-visible and fourier transform infrared spectroscopy," J. Biol. Chem., vol. 283, no. 50, pp. 35033-35041, 2008. 
[87] M.-K. Neumann-Verhoefen, K. Neumann, C. Bamann, I. Radu, J. Heberle, E. Bamberg, and J. Wachtveitl, "Ultrafast infrared spectroscopy on channelrhodopsin-2 reveals efficient energy transfer from the retinal chromophore to the protein," J. Am. Chem. Soc., vol. 135, 2013.

[88] O. P. Ernst, P. A. S. Murcia, P. Daldrop, S. P. Tsunoda, S. Kateriya, and P. Hegemann, "Photoactivation of channelrhodopsin," J. Biol. Chem., vol. 283, p. $1637,12008$.

[89] P. Hegemann, S. Ehlenbeck, and D. Gradmanny, "Multiple photocycles of channelrhodopsin," Biophys. J., vol. 89, pp. 3911-3918, 2005.

[90] K. Stehfest and P. Hegemann, "Evolution of the channelrhodopsin photocycle model," ChemPhysChem, vol. 11, pp. 1120-1126, 2010.

[91] K. Nikolic, N. Grossman, M. S. Grubb, J. Burrone, C. Toumazou, and P. Degenaar, "Photocycles of channelrhodopsin-2," Photochem. Photobiol., vol. 85, pp. 400-411, 2009.

[92] S. S. Talathi, P. R. Carney, and P. P. Khargonekar, "Control of neural synchrony using channelrhodopsin-2: a computational study," J. Comput. Neurosci., 2011.

[93] F. Schneider, C. Grimm, and P. Hegemann, "Biophysics of channelrhodopsin," Annu. Rev. Biophys., 2015.

[94] J. Y. Lin, "A user's guide to channelrhodopsin variants: features, limitations and future developments," Experimental Physiology, vol. 96, pp. 19-25, 2010.

[95] S. Kleinlogel, K. Feldbauer, R. E. Dempski, H. Fotis, P. G. Wood, C. Bamann, and E. Bamberg, "Ultra light-sensitive and fast neuronal activation with the Ca2+-permeable channelrhodopsin CatCh," Nat. Neurosci., 2011.

[96] E. S. Boyden, F. Zhang, E. Bamberg, G. Nagel, and K. Deisseroth, "Millisecond-timescale, genetically targeted optical control of neural activity," Nat. Neurosci., vol. 8, no. 9, pp. 1263-1268, 2005.

[97] J. Y. Lin, M. Z. Lin, P. Steinbach, and R. Y. Tsien, "Characterization of engineered channelrhodopsin variants with improved properties and kinetics," Biophys. J., vol. 96, pp. 1803-1814, 2009.

[98] V. A. Lórenz-Fonfría, T. Reslera, N. Krause, M. Nack, M. Gossing, G. F. von Mollard, C. Bamann, E. Bamberg, R. Schlesinger, and J. Heberle, "Transient protonation changes in channelrhodopsin-2 and their relevance to channel gating," PNAS, vol. 18, 2013.

[99] H. E. Kato, F. Zhang, O. Yizhar, C. Ramakrishnan, T. Nishizawa, K. Hirata, J. Ito, Y. Aita, T. Tsukazaki, S. Hayashi, P. Hegemann, A. D. Maturana, R. Ishitani, K. Deisseroth, and O. Nureki, "Crystal structure of the channelrhodopsin light-gated cation channel," Nature, 2012. 
[100] H. C. Watanabe, K. Welke, D. J. Sindhikara, P. Hegemann, and M. Elstner, "Towards an understanding of channelrhodopsin function: Simulations lead to novel insights of the channel mechanism," J. Mol. Biol., 2013.

[101] F. Bretschneider and J. R. De Weille, Introduction to electrophysiological methods and instrumentation. Academic Press, 2006.

[102] S. Mittman, D. G. Flaming, D. R. Copenhagen, and J. H. Belgurn, "Bubble pressure measurement of micropipet tip outer diameter," J. Neurosci. Methods, vol. 22, pp. 161-166, 1987.

[103] C. L. Bowman and A. M. Ruknudin, "Quantifying the geometry of micropipets," Cell Biochem. Biophys., vol. 31, no. 2, pp. 185-206, 1999.

[104] J. E. Hall, "Access resistance of a small circular pore," J. Gen. Physiol., vol. 66, no. 4, pp. 531-532, 1975.

[105] L. Maguire, S. Szilagyi, and R. Scholten, "High performance laser shutter using a hard disk drive voice-coil actuator," Rev. Sci. Instrum., vol. 75, p. 3077, 9 2004.

[106] R. E. Scholten, "Enhanced laser shutter using a hard disk drive rotary voicecoil actuator," Rev. Sci. Instrum., vol. 78, no. 2, p. 026101, 2007.

[107] A. D. Edelstein, M. A. Tsuchida, N. Amodaj, H. Pinkard, R. D. Vale, and N. Stuurman, "Advanced methods of microscope control using $\mu$ Manager software," J. Biol. Methods, vol. 1, no. 2, 2014.

[108] S. F. Traynelis, "Software-based correction of single compartment series resistance errors," J. Neurosci. Methods, vol. 86, pp. 25-34, Dec 1998.

[109] J. Hogins, D. C. Crawford, C. F. Zorumski, and S. Mennerick, "Excitotoxicity triggered by neurobasal culture medium.," PLoS ONE, vol. 6, no. 9, p. e25633, 2011.

[110] P. Novak, C. Li, A. I. Shevchuk, R. Stepanyan, M. Caldwell, S. Hughes, T. G. Smart, J. Gorelik, V. P. Ostanin, M. J. Lab, G. W. J. Moss, G. I. Frolenkov, D. Klenerman, and Y. E. Korchev, "Nanoscale live-cell imaging using hopping probe ion conductance microscopy," Nat. Methods, vol. 6, pp. 279 - 281, 2009.

[111] D. Klenerman, A. Shevchuk, P. Novak, Y. E. Korchev, and S. J. Davis, "Imaging the cell surface and its organization down to the level of single molecules," Philos Trans R Soc Lond B Biol Sci, vol. 368, p. 20120027, Feb 2013.

[112] I. Horcas, R. Fernández, J. M. Gómez-Rodríguez, J. Colchero, J. GómezHerrero, and A. M. Baro, "WSXM: A software for scanning probe microscopy and a tool for nanotechnology," Rev. Sci. Instrum., vol. 78, no. 1, 2007.

[113] D. Nečas and P. Klapetek, "Gwyddion: an open-source software for SPM data analysis," Cent. Eur. J. Phys, vol. 10, pp. 181-188, 2012. 
[114] K. S. Cole and J. W. Moore, "Potassium ion current in the squid giant axon: dynamic characteristic," Biophys. J., vol. 1, no. 1, pp. 1-14, 1960.

[115] J. H. Holland, Adaptation in natural and artificial systems: an introductory analysis with applications to biology, control, and artificial intelligence. MIT press, 1992.

[116] T. Bäck, "Optimal mutation rates in genetic search," in Proceedings of the fifth International Conference on Genetic Algorithms, pp. 2-8, Morgan Kaufmann, 1993.

[117] G. Ochoa, I. Harvey, and H. Buxton, "Optimal mutation rates and selection pressure in genetic algorithms.," in GECCO, pp. 315-322, Citeseer, 2000.

[118] J. Stender, E. Hillebrand, and J. Kingdon, Genetic algorithms in optimisation, simulation and modelling, vol. 23. IOS Press, 1994.

[119] A. V. Andrade, L. d. Errico, A. L. L. d. Aquino, L. P. d. Assis, and C. H. N. d. R. Barbosa, "Analysis of selection and crossover methods used by genetic algorithm-based heuristic to solve the lsp allocation problem in mpls networks under capacity constraints," 2008.

[120] C. Schmidt-Hieber and J. Bischofberger, "Fast sodium channel gating supports localized and efficient axonal action potential initiation," J. Neurosci., vol. 30, no. 30, pp. 10233-10242, 2010. 


\section{Acknowledgments}

First of all I want to thank my supervisor Christoph Schmidt for giving me the opportunity to work on such interesting projects and for giving me advice and pointing out the best way to proceed on a regular basis. Then I want to thank Andreas Neef for helping devise both the AIS and ChR projects, teaching me most of what I know about electrophysiology, helping me design much of my custom software and much more. Without him much of this work would not have been possible. I also want to thank the third member of my thesis committee Tobias Moser for his opinions and advice, especially in regard to channelrhodopsins. Then I want to thank Fred Wolf for providing the initial idea and theory for the project of investigating the AIS as well as Andres Agudelo-Toro for his patients in explaining the workings of the necessary FEM simulation. Then I want to thank Natalie Elia for teaching me about the abscission process and for her hospitality. Christopher Dilip deserves my thanks for preparing many of the neuron cultures. I also want to thank the people of the 'MPI for experimental medicine' for their help and for providing many of the cells I needed for my work. I want to specifically mention Ricardo Martins Merino for his help and advice on electrophysiological measurements.

I want to thank Florian Rehfeld and Dieter Klopfenstein for their advice.

I want to thank the mechanical and electronic workshops for their fast and reliable work, as well as Thomas Geiling for his IT work. I also want to thank our technical assistants Kerstin von Roden, Tanja Gall, Ulrike Schulz and our secretaries Elke Zech, Sabine Huhnold, Nicole Rehbein and Ursula Hahn-Wörgötter without whom the institute would most likely stop functioning within hours.

Then I want to thank my current and former $\mathrm{PhD}$ colleagues for their help, the great work environment and all the fun we had outside of work.

Last but not least I want to thank my family and friends as well as my girlfriend for their support during the course of my PhD, especially during the last months. 
Curriculum Vitae

\section{Ulrich Arthur Fromme}

Waldweg 12

37073 Göttingen

Germany
Tel.:0551/ 3913830

UFromme@physik3.gwdg.de

Date of birth: $\quad 20.06 .1984$ in Engelskirchen.

Nationality: German

2012-Present $\quad \mathrm{PhD}$-student at Georg-August-Universität Göttingen. Thesis: "Investigation of voltage- and light-sensitive ion channels" under supervision of Prof. Dr. Christoph Schmidt at the 'Third Institute of Physics - Biophysics'.

2009-2011 Master-student at Phillips-Universität Marburg in physics. Thesis: "High Power THz Generation in Lithium Niobate using a Cherenkov-Type geometry" at the institute of 'Experimental Semiconductor Physics' under the supervision of Prof. Dr. Martin Koch.

2005-2009 Bachelor-student at Phillips-Universität Marburg in physics with focus on biology.

Thesis: "Statistische Physik der bakteriellen Zellteilung" at the institute of 'Complex Systems' under supervision of Prof. Dr. Peter Lenz.

2004-2005 Social service at 'Kraichgauheim Bad-Schönborn'

1998-2004 Student at the 'Copernicus-Gymnasium Philippsburg'. Finished with degree 'Abitur'.

1995-1998 Student at the 'German School Washington DC'.

1990-1994 Student at 'Wilhelm-Busch Grundschule Waghäusel'. 